\title{
Mikrostruktur und Wachstum bei der ionenstrahlunterstützten Deposition von Yttrium- stabilisierten Zirkonoxid-Filmen
}

\author{
Dissertation \\ zur Erlangung des Doktorgrades \\ der Mathematisch-Naturwissenschaftlichen Fakultäten \\ der Georg-August-Universität zu Göttingen
}

vorgelegt von

Lars-Oliver Kautschor

aus Stade

Göttingen 2002 
D 7

Referent:

Prof. Dr. H. C. Freyhardt

Korreferent:

P. D. Dr. M. Seibt

Tag der mündlichen Prüfung: $\quad$ 22.11.2002 


\section{Inhalt}

$1 \quad$ Einleitung......................................................................................................................................... 1

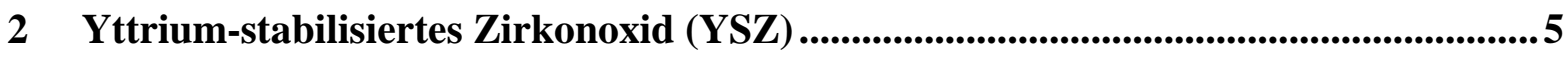

$3 \quad$ Filmherstellung ...........................................................................................................................6

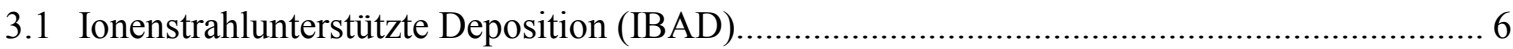

3.2 Depositionsanlage und Depositionsbedingungen ............................................................... 7

4 Charakterisierungsmethoden ..................................................................................10

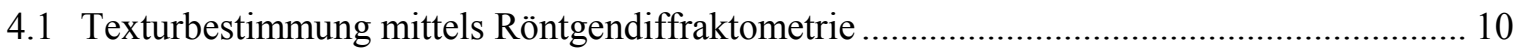

4.2 Netzebenenabbildung und Fast Fourier-Transformation.................................................... 12

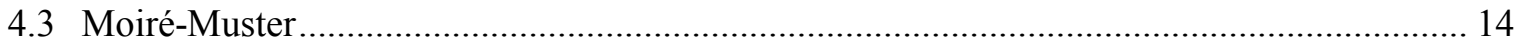

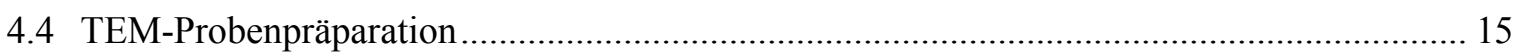

5 Wachstum und Mikrostruktur von IBAD-YSZ-Schichten....................................19

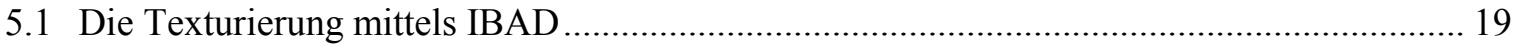

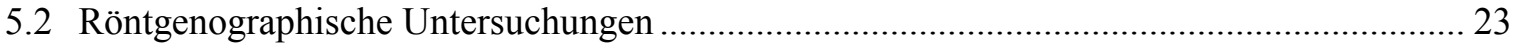

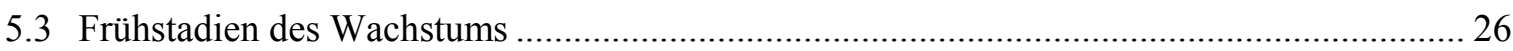

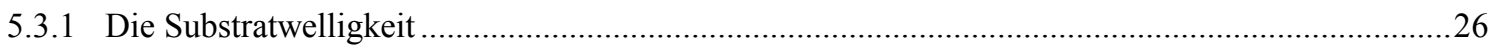

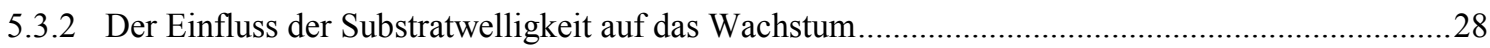

5.3.3 Einfluss der Stromdichte des unterstützenden Ionenstrahls auf die Anfangsschicht ......................31

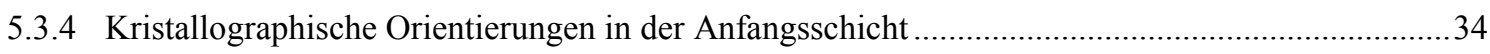

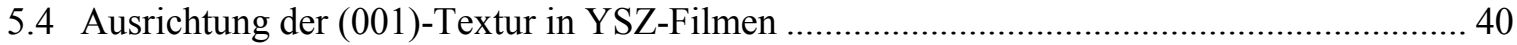

5.4.1 Entwicklung der Texturen in unter IBAD-Bedingungen abgeschiedenen YSZ-Filmen ..................40

5.4.2 Einfluss der Stromdichte des unterstützenden Ionenstrahls.....................................................45 
6 Hochauflösende Untersuchungen zur Texturausbildung .......................................48

6.1 Kontinuierliche Orientierungsänderung während des Wachstums ......................................... 49

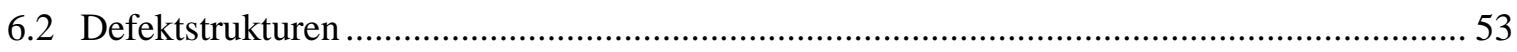

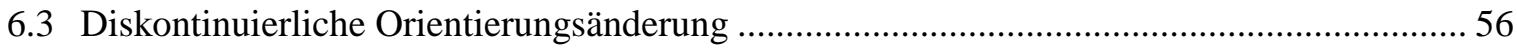

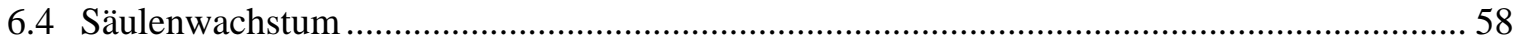

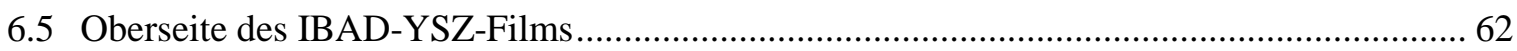

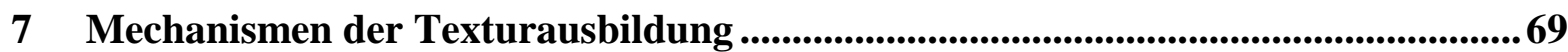

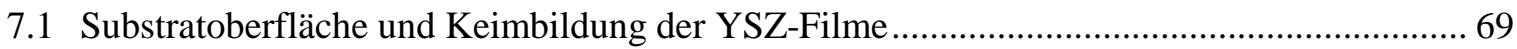

7.2 Einfluss des unterstützenden Ionenstrahls auf die Keimbildung in YSZ-Filmen im

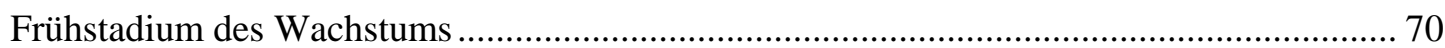

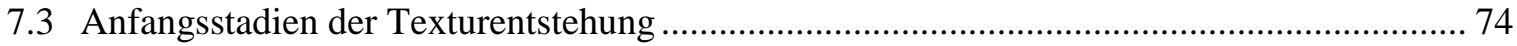

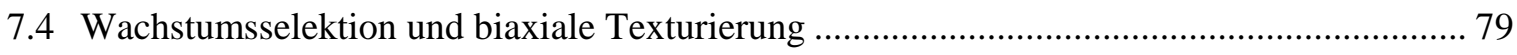

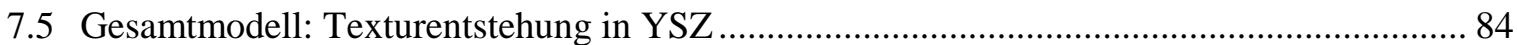

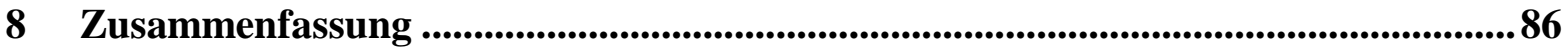

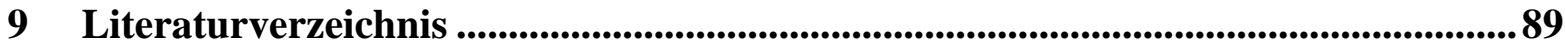

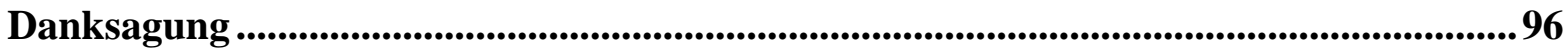

Lebenslauf...............................................................................................................................................97 


\section{Einleitung}

Ursprünglich wurden Ionenquellen als Antriebsaggregate für Satelliten entwickelt. Für die Herstellung dünner Schichten in Vakuumdepositionsanlagen können Ionenquellen aber auch zur Zerstäubung eines Targets eingesetzt werden. Das abgetragene Material kann dann auf einem Substrat abgeschieden werden. In den letzten Jahrzehnten sind insbesondere sogenannte Kaufman-Ionenquellen in der Dünnschichttechnologie eingesetzt worden. Sie erlauben die Erzeugung eines Ionenstrahls mit weitgehend paralleler Trajektorie der Ionen, mit definierter Stromdichte und vorgegebener Teilchenenergie, um so zum Beispiel gezielt Oberflächen- und Schichteigenschaften zu modifizieren. Durch den Ionenbeschuss lassen sich vorgegebene Oberflächen z.B. vergüten, strukturieren oder Halbleiter dotieren um nur einige Anwendungsbeispiele zu nennen.

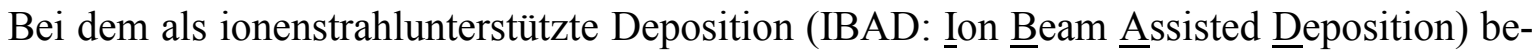
zeichneten Verfahren ist ein Ionenstrahl während des Depositionsprozesses auf die wachsende Schicht gerichtet. Auf diese Weise lassen sich Funktionsschichten mit besonderen Eigenschaften herstellen. So ist es zum Beispiel möglich, die Textur einer Schicht durch den unterstützenden Ionenstrahl gezielt zu beeinflussen. Yu und Mitarbeiter zeigten 1985 erstmals, dass sich beim Wachstum von Niob-Filmen eine biaxiale Textur ausbildet, wenn ein unterstützender Ionenstrahl unter einem bestimmten Winkel auf die Schicht gerichtet ist [Yu et al., 1985]. Bei der biaxialen Textur wird dabei zwischen der Textur in Richtung der Substratnormalen (out-of-plane-Textur) und der in der Ebene (in-plane-Textur) unterschieden.

Ein wichtiges Einsatzgebiet der biaxiale Zwangstexturierung mittels der ionenstrahlunterstützten Deposition ist der Herstellung von Pufferschichten für epitaktisch abgeschiedene Hochtemperatursupraleiter (HTSL), wie z.B. $\mathrm{YBa}_{2} \mathrm{Cu}_{3} \mathrm{O}_{7-\delta}$ (YBCO). Dieser Supraleiter ist für die Herstellung von Bandleitern der zweiten Generation sehr geeignet. Kritische Stromdichten von mehr als $10^{6} \mathrm{~A} / \mathrm{cm}^{2}(77 \mathrm{~K}, 0 \mathrm{~T}$ ) ermöglichen einen Transport von hohen Strömen in diesen Leitern [Wu et al., 1995 / Freyhardt et al., 1996 / Betz et al., 1997 / Bauer et al., 1999].

Neben der Anisotropie der supraleitenden Eigenschaften von YBCO führt vor allem die kurze Kohärenzlänge der supraleitenden Ladungsträger dazu, dass z.B. Korngrenzen die Stromtragfähigkeit drastisch reduzieren. So nimmt die kritische Stromdichte über eine Korngrenze mit einem Verkippungswinkel $\geq 5^{\circ}$ um bis zu 90\% ab [Dimos et al., 1990]. Um den Anteil an Großwinkelkorngrenzen im YBCO möglichst gering zu halten, muss dem YBCO 
eine über große Längen epitaxiefähige Unterlage mit wenigen Großwinkelkorngrenzen zur Verfügung stehen.

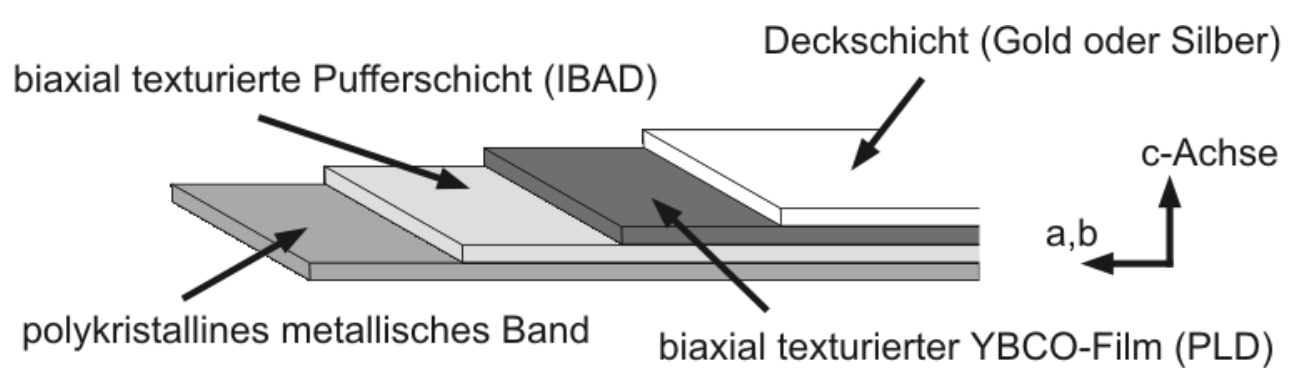

Abbildung 1.1: Mögliche Schichtfolge eines Bandleiters der zweiten Generation

In Abbildung 1.1 ist der prinzipielle Aufbau eines Bandleiters der zweiten Generation dargestellt. Auf einem flexiblen metallischen Band wird eine sogenannte Pufferschicht, die als Diffusionsbarriere dient, abgeschieden. In zwei weiteren Prozessschritten werden dann die eigentliche supraleitende Schicht (YBCO, z.B. mittels PLD: Pulsed Laser Deposition) und eine Deckschicht, die zum Schutz und der Kontaktierung des Supraleiters dient, deponiert. Durch ein epitaktisches Wachstum auf der Pufferschicht erreicht man den gewünschten geringen Anteil an Großwinkelkorngrenzen im Supraleiters. Durch eine IBAD-Deposition kann die Pufferschicht die notwendige ausgeprägte Textur aufweisen, so dass das metallische Band polykristallin sein kann. Alternativ dazu kann auch das metallische Substrat (z.B. Ni- und NiLegierungen) durch einen Walz- und Rekristallisationsprozess biaxial texturiert werden

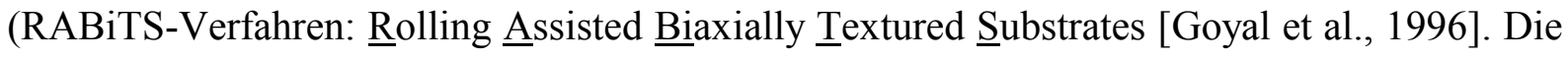
Pufferschicht muss dann epitaktisch abgeschieden werden, um den gewünschten Texturübertrag auf den Supraleiter zu gewährleisten.

Iijima und Mitarbeiter stellten 1991 erste Ergebnisse von mittels ionenstrahlunterstützter Deposition (IBAD) hergestellter biaxial texturierter Filme aus kubischem Yttriumstabilisierten Zirkonoxid (YSZ) vor [Iijima et al., 1991]. Sie richteten den unterstützenden Ionenstrahl während der Deposition unter einem Winkel von $55^{\circ}$ zur Substratnormalen auf den aufwachsenden Film. Dadurch richtet sich eine $<111>$-Richtung des YSZ parallel zum unterstützenden Ionenstrahl aus, bei gleichzeitigem (001)-Wachstum. Mit dieser Methode können texturierte YSZ-Filme auf polykristallinen bzw. amorphen Substraten abgeschieden werden und ermöglichen dadurch die Herstellung von supraleitenden Filmen mit guten kritischen Eigenschaften auch auf an eine technische Anwendung angepassten Substraten (polykristalline metallische Bänder, keramische Platten etc.). 
Im Gegensatz zu MgO, das schon bei Schichtdicken von ca. $20 \mathrm{~nm}$ eine ausgeprägte Textur aufweist [Wang et al., 1997], erfolgt die Verbesserung der in-plane-Textur in YSZ-Filmen über einen Ausleseprozess während des Wachstums, so dass Schichtdicken von ca. $1 \mu \mathrm{m}$ benötigt werden um ausgeprägte Texturen zu erhalten [Wiesmann, 1998 / Dzick, 2000].

Der zugrundeliegende Mechanismus der Texturausbildung unter Ionenbeschuss ist aber bis heute nicht vollständig verstanden. Es existieren Modelle, die die Texturentwicklung unter bestimmten Depositionsbedingungen beschreiben. So beschreibt das Modell von Sonnenberg und Mitarbeitern, dass bei der Abscheidung von YSZ mittels Elektronenstrahlverdampfung auf geheizten Substraten $\left(600^{\circ} \mathrm{C}\right)$ unter Verwendung eines unterstützenden Ionenstrahls durch selektives Ätzen ein Abschatten benachbarter Körner resultiert. Die Texturausbildung geschieht durch das Überwachsen fehlorientierter Körner [Sonnenberg et al., 1993]. Die vorhergesagte Porosität wird in IBAD-YSZ-Filmen, die unter diesen Bedingungen abgeschieden wurden, auch tatsächlich beobachtet. Dagegen erhält man bei einer niedrigen Substrattemperatur sehr dichte Filme, wenn man Sputtertechniken zur Abscheidung der Filme einsetzt.

Ein weiteres Modell, das die Texturverbesserung mit wachsender Filmdicke beschreibt, wurde von Bradley und Harper unter der Annahme vorgestellt, dass unter Ionenbeschuss anisotrope Ätzraten auftreten. Zum unterstützenden Ionenstrahl fehlorientierte Körner werden während des Wachstums stärker abgetragen und somit überwachsen. Die anisotropen Ätzraten können durch den Channeling-Prozess beschrieben werden [Bradley und Harper, 1988]. Dieses Modell wurde entwickelt, um das Wachstum der von Yu und Mitarbeitern hergestellten IBAD-Niob-Filmen zu erklären [Yu et al., 1985 / Yu et al., 1986]. Die von Iijima und Mitarbeitern beobachtete Texturverbesserung mit zunehmender Filmdicke [Iijima et al., 1998] lässt sich ebenfalls mit diesem Modell beschreiben. Allerdings erhalten sie schon zu Beginn des Wachstums eine (001)-Textur, welches nicht mit dem Modell von Bradley und Harper erklärt werden kann.

Dzick geht in seinem Modell von einer spannungsinduzierten Bildung einer dünnen drahttexturierten (110)-Keimschicht aus. Im weiterem Verlauf bewirkt der Ionenstrahl einerseits ein homoepitaktisches Wachstum, andererseits kann auf (110)-orientierten Körnern aber auch ein epitaktisches Wachstum einer (001)-Orientierung einsetzen. Nach Dzick [Dzick, 2000] tritt letzteres auf, wenn ein (110)-orientiertes Korn so zum Ionenstrahl orientiert ist, dass ein epitaktisch aufwachsendes (001)-Korn seine $<111>$-Richtung parallel zum einfallenden Ionenstrahl ausrichtet. In diesem Modell entsteht die biaxiale (001)-Textur des YSZ sprunghaft.

Im Mittelpunkt dieser Arbeit stehen Untersuchungen der Struktur und Texturausbildung in YSZ-Filmen mittels der Transmissionselektronenmikroskopie (TEM). Ziel ist es dabei ein detaillierteres Bild der Veränderung der Mikrostruktur mit zunehmender Filmdicke zu entwickeln und solche Prozesse zu identifizieren, die zur Einstellung der biaxialen Textur führen. 
Die Kenntnis solcher Prozesse bildet eine wesentliche Voraussetzung für die Entwicklung neuer Wachstumsmodelle, die eine verbesserte Beschreibung der Texturauslese bei ionenstrahlunterstützten Depositionsprozessen ermöglichen.

Im einzelnen ist die vorliegende Arbeit wie folgt gegliedert: Zunächst sollen die Struktur des YSZ und die YSZ-Filmherstellung beschrieben werden. Auf eine Darstellung der Charakterisierungsmethoden folgt im Abschnitt 5 eine Beschreibung des Wachstums und der Mikrostruktur von YSZ-Filmen. Abschnitt 6 behandelt die hochauflösenden TEM-Untersuchungen. Die Ergebnisse werden in Abschnitt 7 mit verschiedenen Modellvorstellungen zum Wachstum verglichen. 


\section{Yttrium-stabilisiertes Zirkonoxid (YSZ)}

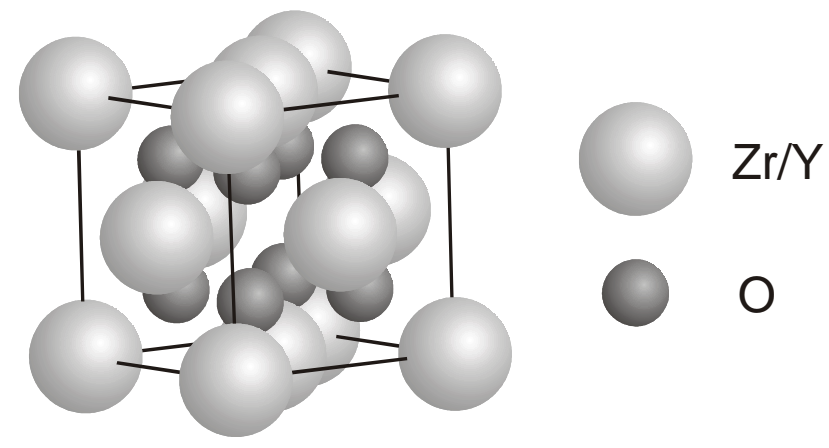

Abbildung 2.1: Darstellung der $\mathrm{CaF}_{2}$-Struktur des kubischen YSZ

Für ein epitaktisches c-achsen-orientiertes Wachstum von YBCO auf Yttriumstabilisiertem Zirkonoxid (im folgenden: YSZ) ist dessen kubische Hochtemperaturphase erforderlich (Raumgruppe: Fm3m, s. Abbildung 2.1). Während reines $\mathrm{ZrO}_{2}$ in der tetragonalen, der monoklinen oder der orthorhombischen Kristallstruktur auftritt [Heuer et al., 1985], ist es durch Zusatz von $\geq 8$ mol\% $\mathrm{Y}_{2} \mathrm{O}_{3}$ möglich, die kubische $\mathrm{CaF}_{2}$-Struktur bei Raumtemperatur zu stabilisieren [Scott, 1975]. Die Gitterkonstante dieser Struktur variiert je nach $\mathrm{Y}_{2} \mathrm{O}_{3}$-Gehalt und beträgt bei 8 mol\% 5,13 Å [Dzick, 2000].

Sowohl die Gitterkonstante als auch die Dichte von ca. 6 g/ $\mathrm{cm}^{3}$ [Wiesmann, 1994] vergröBern sich mit steigendem Yttrium-Gehalt. Der thermische Ausdehnungskoeffizient zwischen Raumtemperatur und $800^{\circ} \mathrm{C}$ liegt bei $10^{-5} \mathrm{~K}^{-1}$ [Scubican et al., 1978] und entspricht annähernd dem Ausdehnungskoeffizienten von YBCO [Meingast et al., 1991]. YSZ zeichnet sich durch eine schlechte Wärmeleitfähigkeit aus und ist ein Isolator.

Der Yttrium-Zusatz verursacht strukturelle Sauerstoffleerstellen, so fehlt zum Beispiel bei einem 8 mol\% $\mathrm{Y}_{2} \mathrm{O}_{3}$-Zusatz jedes 27te Sauerstoffatom. Diese Eigenschaft macht YSZ zu einem guten Sauerstoffionenleiter [Hund, 1951]. 


\section{Filmherstellung}

Die in dieser Arbeit untersuchten YSZ-Filme wurden mittels der ionenstrahlunterstützten Deposition hergestellt. So sollen in diesem Abschnitt die für die Filmherstellung benutzte Depositionskammer und die wichtigsten Depositionsparameter beschrieben werden.

\subsection{Ionenstrahlunterstützte Deposition (IBAD)}

Bei der ionenstrahlunterstützten Deposition, im folgenden auch IBAD (Ion Beam $\underline{\text { Assisted }}$ Deposition), wird während der Deposition ein unterstützender Ionenstrahl niedriger Energie (ca. $300 \mathrm{eV}$ ) auf das Substrat gerichtet (vgl. Abbildung 3.1). Auf diese Weise wird das Wachstum des Films so beeinflusst, dass sich bei bestimmtem Einfallswinkel des unterstützenden Ionenstrahls kristallographische Richtungen des aufwachsenden Materials parallel zum unterstützenden Ionenstrahl ausrichten. Der optimale Einfallswinkel variiert je nach Textur und Material.

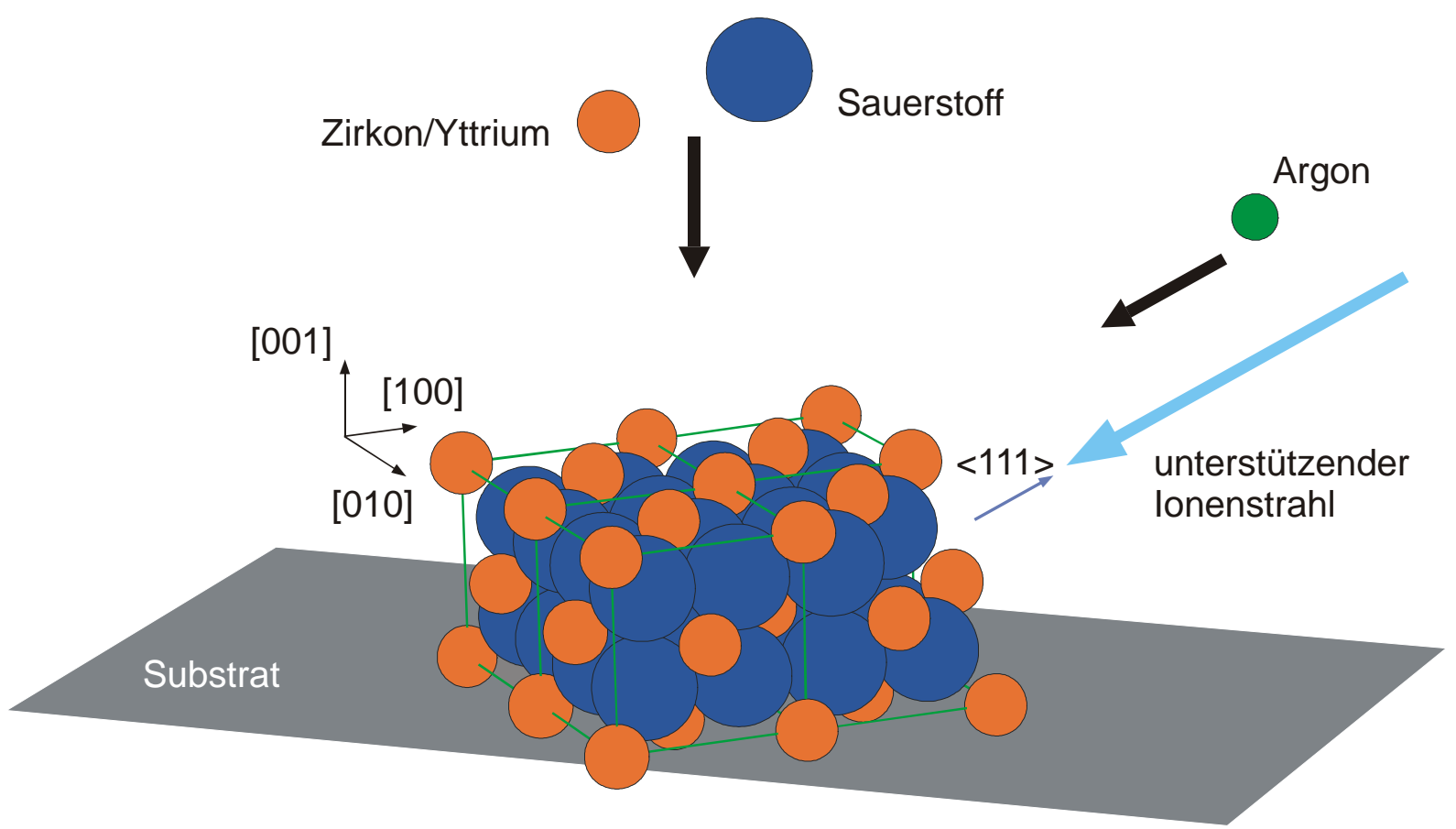

Abbildung 3.1: Schematische Darstellung zum Verständnis des IBAD-Prozesses 
Bei (001)-orientierten IBAD-YSZ-Filmen wird eine optimale Ausbildung einer in-planeTextur bei einem Einschusswinkel von ca. $55^{\circ}$ beobachtet. Dieses entspricht dem Winkel zwischen den $<001>$ - und $<111>$-Ebenennormalen im kubischen System $\left(54,7^{\circ}\right.$, vgl. Abbildung 3.1). Verringert man den Winkel, so nimmt die Textur ab, bis man schließlich drahttexturierte (001)-orientierte Filme erhält. Vergrößert man den Winkel, so erfolgt bei ca. $65^{\circ}$ ein Umschlagen des (001)- in ein (111)-Wachstum [Dzick, 1996 / Mao et al., 1997 / Iijima et al., 1998].

Neben dem Einfallswinkel ist die Ionenstrahldichte ein wichtiger Parameter. So wird bei IBAD-YSZ-Filmen, die bei niedriger Dichte des unterstützenden Ionenstrahls mit ArgonIonen (75 eV und $300 \mathrm{eV}$ ) deponiert werden, statt der Ausrichtung der $<111>$-Richtung die Ausrichtung einer $<110>$-Richtung zum unterstützenden Ionenstrahl beobachtet [Sonnenberg et al., 1993 / Kaufman et al., 1998]. Zur Charakterisierung der Beschussintensität verwendet man das sogenannte $\eta$-Verhältnis:

$$
\eta=\frac{\text { Ionenauftreffrate }}{\text { Teilchendepositionsrate }}=\frac{J \cdot V_{m o l}}{N_{A} \cdot e \cdot R}
$$

mit: $J$, Stromdichte des unterstützenden Ionenstrahls, $V_{m o l}$, Molmasse von YSZ, $N_{\mathrm{A}}$, Avogardozahl, $R$, der YSZ-Aufwachsrate ohne unterstützenden Ionenstrahl und Elementarladung $e$.

Verwenden die unterschiedlichen Arbeitsgruppen Ionenenergien von $250-350 \mathrm{eV}$, so variiert das $\eta$-Verhältnis stark und liegt zwischen 0,5 und 3,7, um optimale Texturausbildungen zu erhalten [Wiesmann, 1998].

\subsection{Depositionsanlage und Depositionsbedingungen}

Die in dieser Arbeit untersuchten YSZ-Filme wurden in einer Vakuumanlage mit einer $11 \mathrm{~cm}-$ Kaufman-Ionenquelle (Fa. Ion Tech inc.) als Sputterquelle durch Abtrag eines YttriumZirkon-Legierungstargets abgeschieden. Die genaue Funktionsweise und der Aufbau der Quelle wurden von H. R. Kaufman beschrieben [Kaufman, 1974 / Kaufman et al., 1982]. Als Sputtergas wurde Xenon verwendet. Für die Erzeugung des unterstützenden Ionenstrahls wurde eine zweite Kaufman-Ionenquelle (11 cm Durchmesser) mit Argon eingesetzt. Die Ionenquellen sind so positioniert, dass sich die Ionenstrahlen nicht kreuzen. In Abbildung 3.2 ist die Depositionskammer schematisch dargestellt. Der unterstützende Ionenstrahl trifft unter einem Winkel von $55^{\circ}$ auf das Substrat, das auf einen Halter aufgeklebt ist. Dieser wurde während der Deposition weder gekühlt noch geheizt. Die Substrattemperatur beträgt ca. $60^{\circ} \mathrm{C}$ [Dzick, 2000 / Sievers, 2002]. 


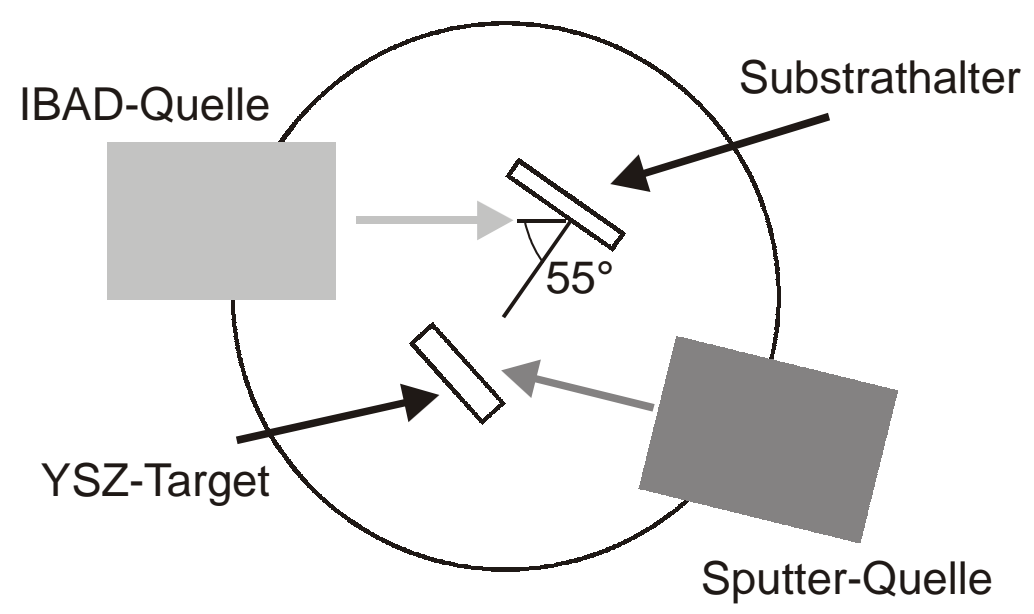

Abbildung 3.2: Schematische Darstellung der IBAD-Anlage in Aufsicht

Vor der Deposition wird der unterstützende Ionenstrahl zur Reinigung des Substrates eingesetzt. Dabei wird das Substrat mit Argonionen ( $400 \mathrm{eV}$, Stromdichte $\geq 300 \mu \mathrm{A} / \mathrm{cm}^{2}, 55^{\circ}$ ) einige Minuten geätzt. So werden Lösungsmittelrückstände und Absorbate an der Oberfläche beseitigt und die Haftung der Filme dadurch deutlich verbessert [Dzick, 2000].

Da bei der Sputterdeposition der Sauerstoff, der aus dem YSZ-Target ausgelöst wird, nicht ausreicht, den Film vollständig $\mathrm{zu}$ oxidieren, wird ein Sauerstoffpartialdruck von $0,8-1,3 \times 10^{-4}$ mbar in der Kammer eingestellt, um so durch ,reaktives Sputtern“ die nachträgliche Oxidation der Filme zu gewährleisten [Wiesmann, 1994 / Dzick, 1996 / Wiesmann, 1998 / Dzick, 2000].

Um den Einfluss des unterstützenden Ionenstrahls zu untersuchen, wurden durch Variation der Stromdichte des unterstützenden Ionenstrahls mit unterschiedlichen $\eta$-Verhältnissen YSZFilme hergestellt. So wird im folgenden zwischen ohne- $(\eta=0)$ und mit Ionenstrahlunterstützung abgeschiedener Filme unterschieden, wobei das $\eta$-Verhältnis von 2 auf 3 bzw. 4 variiert wurde. Die genauen Depositionsparameter sind in Tabelle 3.1 angegeben. 


\begin{tabular}{|c|c|c|c|c|c|}
\hline & \multicolumn{2}{|c|}{ Sputterquelle } & \multicolumn{2}{|r|}{$\underline{\text { IBAD-Quelle }}$} & \multirow[b]{2}{*}{$\begin{array}{l}\text { Aufwachs- } \\
\text { rate } \\
{[\mathrm{nm} / \mathrm{min}]}\end{array}$} \\
\hline & Gas & $\begin{array}{l}\text { Beschleunigungs- } \\
\text { spannung, Strom }\end{array}$ & Gas & $\begin{array}{c}\text { Beschleunigungs- } \\
\text { spannung, } \\
\text { Stromdichte } J\end{array}$ & \\
\hline $\begin{array}{l}\text { ohne Ionen- } \\
\text { strahlunterst. } \\
\qquad(\eta=0)\end{array}$ & Xenon & $\begin{array}{l}1000 \mathrm{eV} \\
30 \mathrm{~mA}\end{array}$ & - & - & $\sim 20$ \\
\hline $\begin{array}{l}\text { mit Ionen- } \\
\text { strahlunterst. } \\
\quad(\eta=2)\end{array}$ & Xenon & $\begin{array}{c}1000 \mathrm{eV} \\
30 \mathrm{~mA}\end{array}$ & Argon & $\begin{array}{c}300 \mathrm{eV} \\
\mathbf{3 0 0} \mu \mathrm{A} / \mathrm{cm}^{2}\end{array}$ & $\sim 17$ \\
\hline $\begin{array}{l}\text { mit Ionen- } \\
\text { strahlunterst. } \\
\quad(\eta=3)\end{array}$ & Xenon & $\begin{array}{l}1000 \mathrm{eV} \\
30 \mathrm{~mA}\end{array}$ & Argon & $\begin{array}{c}300 \mathrm{eV} \\
\mathbf{4 5 0} \mu \mathrm{A} / \mathrm{cm}^{2}\end{array}$ & $\sim 16$ \\
\hline $\begin{array}{l}\text { mit Ionen- } \\
\text { strahlunterst. } \\
\quad(\eta=4)\end{array}$ & Xenon & $\begin{array}{l}1000 \mathrm{eV} \\
30 \mathrm{~mA}\end{array}$ & Argon & $\begin{array}{c}300 \mathrm{eV} \\
\mathbf{6 0 0} \mu \mathrm{A} / \mathrm{cm}^{2}\end{array}$ & - \\
\hline
\end{tabular}

Tabelle 3.1: Depositionsparameter der für diese Arbeit bei verschiedenen $\eta$-Verhältnissen abgeschiedenen YSZ-Filme. Die Aufwachsraten wurden aus Schichtdickenmessungen und TEM-Aufnahmen ermittelt. 


\section{Charakterisierungsmethoden}

Unter dem Begriff „Textur“ versteht man die Gesamtheit der Orientierungen der Kristallite in einem Kristall. Bei biaxial texturierten Filmen ist die Angabe von zwei Texturkomponenten erforderlich. Die Bestimmung der Textur erfolgt mittels Röntgenmessungen. Diese erlauben eine Charakterisierung über makroskopische Probenflächen. Allerdings wird bei den Röntgenmessungen ein über die gesamte Filmdicke gemittelter Wert bestimmt. So beträgt z.B. die Eindringtiefe für YSZ bei einer Co-K $\mathrm{K}_{\alpha}-$ Strahlung $(\lambda=1,7903 \AA)$ ca. 7,4 $\mu \mathrm{m}$ [Wiesmann, 1998]. Mittels der Transmissionselektronenmikroskopie (TEM) sind dagegen ortsaufgelöste Messungen möglich.

\subsection{Texturbestimmung mittels Röntgendiffraktometrie}

Die Röntgendiffraktometrie ist in der Materialphysik eine wichtige Analysetechnik zur Charakterisierung dünne Filme. Mit ihrer Hilfe lassen sich Aussagen über die Phase, Fremdphasen, Ausscheidungen, Gitterkonstanten und Texturen machen. Im Gegensatz zu anderen Analysemethoden (z.B. TEM, SIMS) sind Röntgenanalysen zerstörungsfrei und erfordern auch keine besondere Vorpräparation der Proben.

Bei der Röntgendiffraktometrie wird der einfallende Röntgenstrahl und der Detektor so zueinander positioniert, dass bei geeigneter Orientierung einer kristallinen Probe die BRAGGBedingung

$$
n \cdot \lambda=2 \cdot d_{h k l} \cdot \sin \Theta
$$

erfüllt ist. Dabei ist $d_{h k l}$ der Netzebenenabstand der $(h k l)$-Ebene ( $h k l$ : MiLLERschen Indizes), $n$, die Beugungsordnung (ganze Zahl), $\lambda$ die Wellenlänge der Röntgenstrahlung und $\Theta$, der Winkel, den der einfallende bzw. reflektierte Röntgenstrahl mit den Netzebenen einnimmt.

\section{- Zweikreis-Diffraktometrie, out-of-plane-Textur}

Abbildung 4.1 zeigt ein typisches Röntgendiffraktogramm eines YSZ-Films mit ausgeprägtem (001)-Wachstum. Es wurde mit einem Zweikreis-Diffraktometer D500 der Firma Siemens $\left(\mathrm{CoK}_{\alpha}\right.$-Röhre, $\left.\lambda_{\mathrm{Co}-\mathrm{K \alpha}}=1,7903 \AA\right)$ gemessen. 


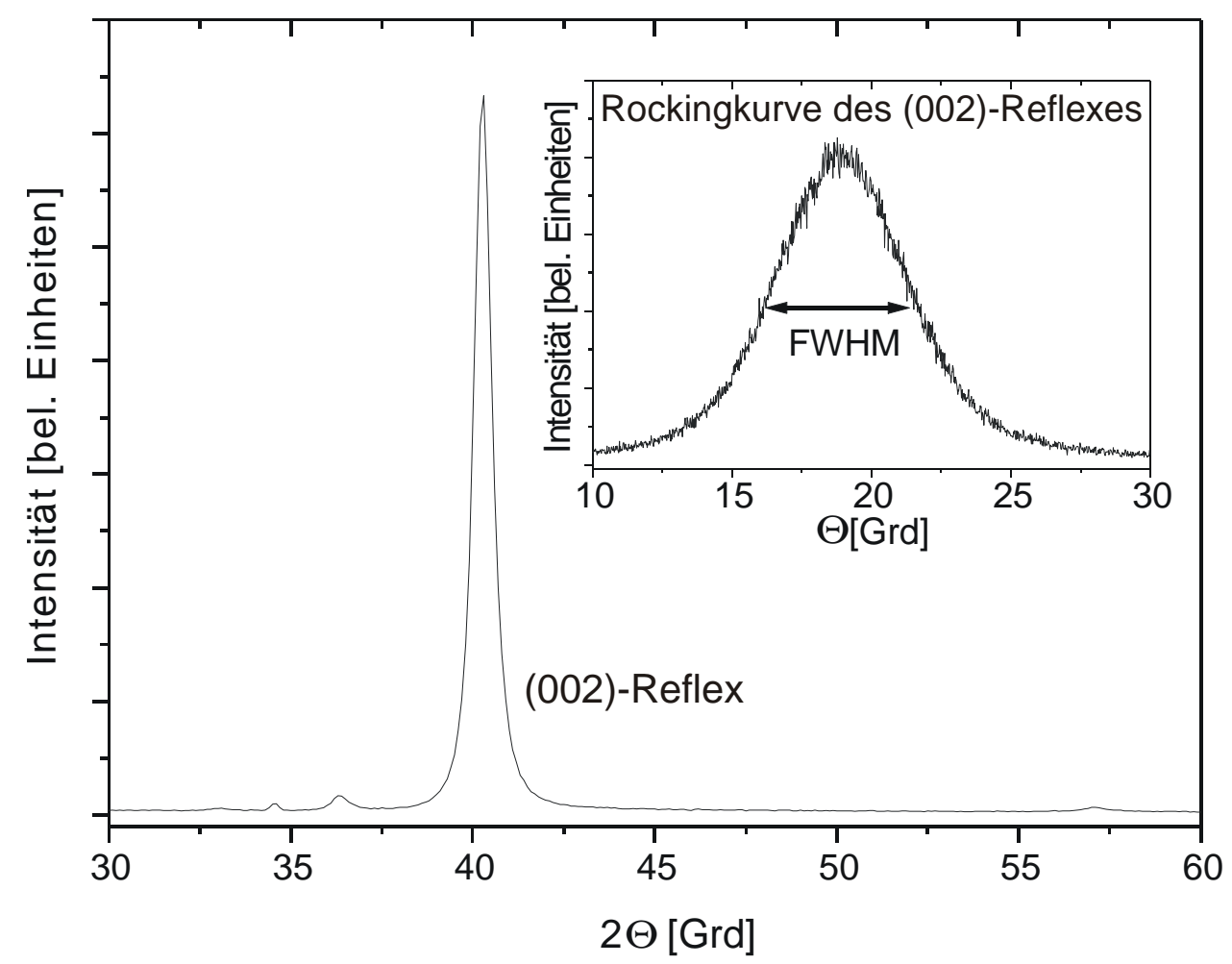

Abbildung 4.1: Röntgendiffraktogramm eines IBAD-YSZ-Films mit ausgeprägtem (001)Wachstum und Rockingkurve des (002)-Reflexes

Misst man die Intensität des (002)-Reflexes als Funktion des Winkels zwischen Probe und einfallenden Röntgenstrahl, so erhält man die sogenannte Rockingkurve. Die mittlere Halb-

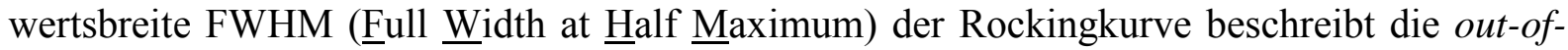
plane-Textur (vgl. Abbildung 4.1) und ist ein Maß für die Breite der Orientierungsverteilung der (001)-orientierten Kristallbereiche.

\section{- Vierkreis-Diffraktometrie, in-plane-Textur}

Für die Texturmessungen wurde das Texturgoniometer D8 Advance (Fa. Bruker AXS) des Zentrums für Funktionswerkstoffe gGmbH, Göttingen verwendet, das ebenfalls mit einer monochromatischen $\mathrm{Co}-\mathrm{K}_{\alpha}$-Strahlung arbeitet. Mit dem Vierkreis-Diffraktometer können Proben so zum Röntgenstrahl positioniert werde, dass alle kristallographischen Richtungen der Probe ausgemessen werden können.

Bei der Bestimmung der in-plane-Textur wird eine bestimmte Reflexlage ( $h k l)$ fest eingestellt. Bei einem kubischen System, wie es beim YSZ der Fall ist, ist dies z.B. der (111)Reflex. Während die Position der Probe auf dem $\phi$-Kreis $\left(0^{\circ}-360^{\circ}\right)$ und dem $\chi$-Kreis $\left(0^{\circ}-\right.$ $90^{\circ}$ ) variiert wird, wird die Röntgenintensität gemessen. Die gemessene Intensität aller Probenorientierungen wird dabei in einer Polfigur (WULFF'sche Projektion) eingetragen, so dass 
der Radius den Kippwinkel $\chi$ und der Drehwinkel $\phi$ die Probenrotation beschreibt. Bei einem kubischen, (001)-orientierten System treten die vier äquivalenten $\{111\}$-Ebenen in der entstandenen Polfigur im Abstand von $\Delta \phi=90^{\circ}$ auf (vgl. Abbildung 4.2 a).

Der oben beschriebene Messaufwand kann erheblich reduziert werden, wenn die out-ofplane-Textur bekannt ist. Falls bei kubischen Materialien die Probe im wesentlichen (001)texturiert ist (vgl. Abbildung 4.1), kann die in-plane-Textur aus der Halbwertsbreite der $\{111\}$-Pole entlang des $\chi=54,7^{\circ}$-Kreises bestimmt werden. Es reicht dann, einen sogenannten $\phi$-Scan zu messen (vgl. Abbildung 4.2 b).
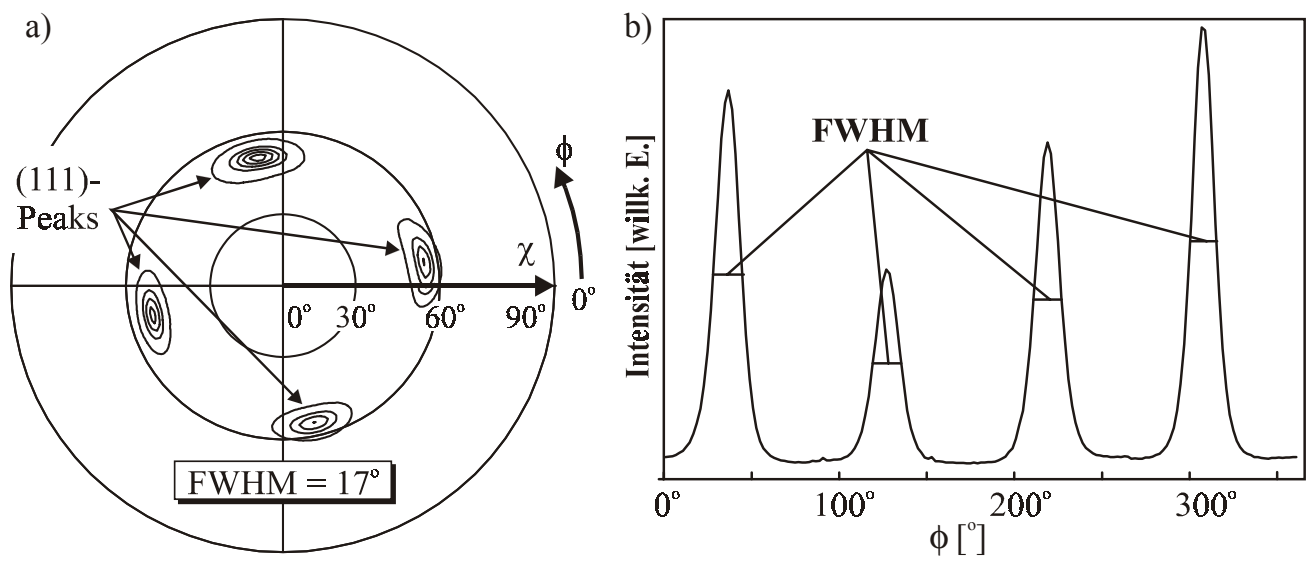

Abbildung 4.2: a) (111)-Polfigur mit den vier <111>-Polen eines in-plane texturierten IBADYSZ-Films, b) $\phi$-Scan durch die vier $\{111\}$-Pole bei $\chi=54,7^{\circ}$, FWHM: Full Width at Half Maximum, Halbwertsbreite (aus: [Dzick, 2000])

\subsection{Netzebenenabbildung und Fast Fourier-Transformation}

Die Mikrostruktur der YSZ-Filme wurde zum einen mittels konventioneller Transmissionselektronenmikroskopie (TEM) an einem Philips 420ST EM (Beschleunigungsspannung: $120 \mathrm{eV}$ ) und zum anderen mittels hochauflösender Transmissionselektronenmikroskopie (HREM) an einem Philips CM200 FEG ${ }^{1}$ (Beschleunigungsspannung: $200 \mathrm{eV}$ ) untersucht.

Hochaufgelöste Elektronenmikroskopiebilder sind Phasenkontrastabbildungen. Die Elektronen werden im Objekt gebeugt und führen zu einem fouriertransformierten Bild in der hinteren Brennebene der Objektivlinse (Beugungsbild). Die Beugungspunkte in der hinteren Brennebene der Objektivblende sind gemäß dem HuYGENS-FrESNELschen Prinzip Ausgangspunkte für Kugelwellen, die als vergrößerte Objektabbildung in der Bildebene interferieren.

\footnotetext{
${ }^{1}$ IV. Physikalische Institut der Universität Göttingen
} 
Dieser Vorgang entspricht mathematisch einer zweiten FOURIER-Transformation. Diese ist jedoch unvollständig, da technisch bedingte Linsenfehler wie sphärische und chromatische Abberation und eine Defokussierung der Objektivlinse den abgebeugten Wellen einen zusätzlichen Phasenfaktor aufprägt [Spence, 1988 / Alexander, 1997].

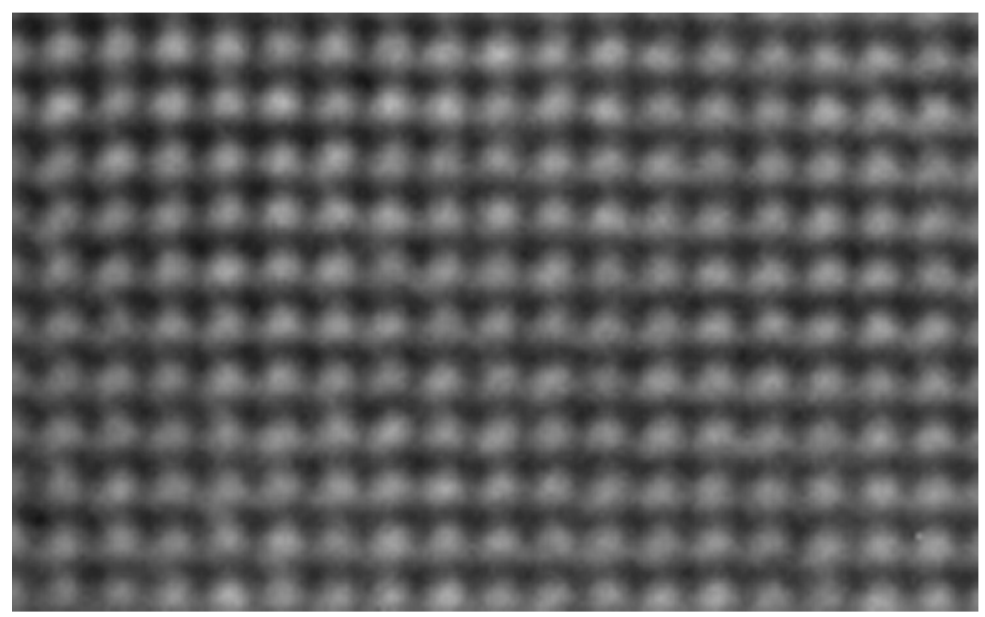

Abbildung 4.3: Hochaufgelöste Aufnahme eines Silizium-Einkristalls. Das Punktmuster gibt die Periodizität der Atomsäulen des Kristalls wieder. Ob helle Punkte dem tatsächlichen Ort der Atome entsprechen, lässt sich nur mit Hilfe von Simulationsrechnungen feststellen.

Im allgemeinen lässt sich der entstandene Bildkontrast (vgl. Abbildung 4.3) nicht intuitiv interpretieren. Mit Modellrechnungen bzw. computersimulierten Bildern kann man die HREM-Bilder vergleichen und anhand der Parameter der Modelle Aussagen über den Kontrast machen.

Mit Hilfe der zweidimensionalen Fourier-Transformation (FFT: Fast Fourier Transformation) lassen sich in Hochauflösungsaufnahmen, auf denen Netzebenen unterschiedlicher Periode und Orientierung zu sehen sind, die Orientierungen von kristallinen Bereichen nachträglich bestimmen. Im folgenden sollen zum einfachen Verständnis die einzelnen Schritte der Bildbearbeitung mittels der Fourier-Transformation schematisch dargestellt werden. Die Auswertung der digitalisierten Hochauflösungsaufnahmen erfolgt mit Standardprogrammen (u.a. Digital Micrograph, Firma Gatan).

Die FFT einer Netzebenenabbildung zeigt ein diskretes Punktmuster (vgl. Abbildung 4.4). Die einzelnen Punkte werden wie die eines Beugungsbildes indiziert. Dadurch ist es möglich, lokale Orientierungen zu bestimmen. 


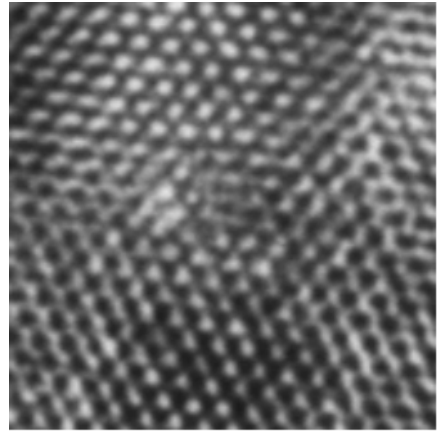

HREM-Bild

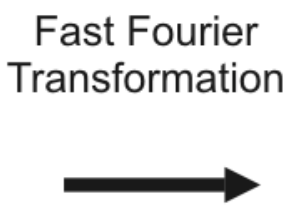

Abbildung 4.4: Netzebenenabbildung mit der dazugehörigen Fourier-Transformation. Die Reflexe werden wie bei einem Beugungsbild indiziert.

Neben der Orientierungsbestimmung lassen sich durch Auswahl bestimmter Reflexe in der FFT einzelne Ebenenscharen herausfiltern (Bragg-Filterung). Dabei werden ein oder mehrere Reflexpaare mittels einer Maske separiert und zurücktransformiert (inverse FFT, vgl. Abbildung 4.5). Auf diese Weise lassen sich z.B. mögliche Defekte besser darstellen.
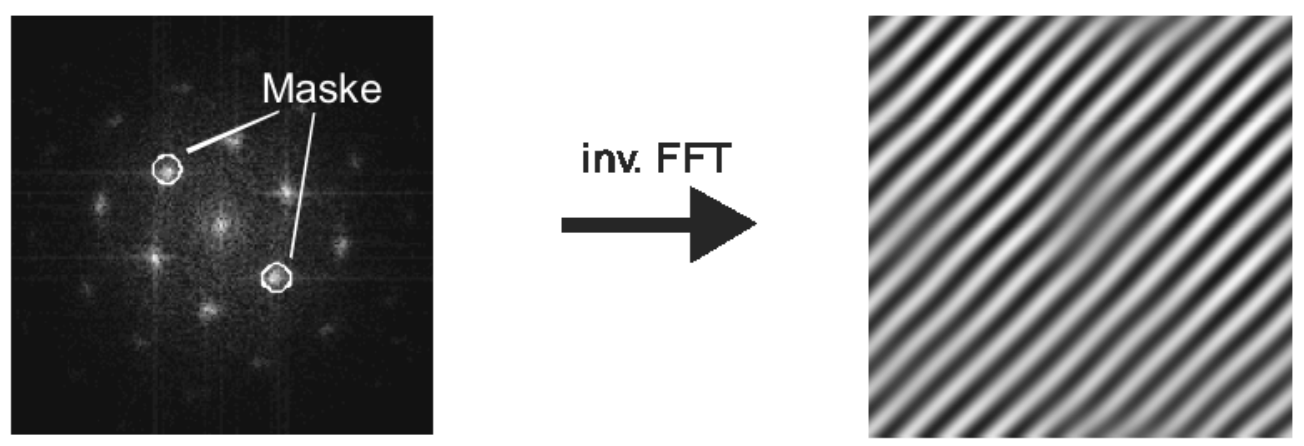

Abbildung 4.5: Mit Hilfe von Masken lassen sich einzelne Reflexe der FFT zurücktransformieren. Man erhält so die Netzebenen, die die Reflexe erzeugen.

\subsection{Moiré-Muster}

Ein in den hier untersuchten Proben häufig beobachteter Kontrast ist das MoIRÉ-Muster. Es entsteht, wenn z.B. zwei Kristallite mit unterschiedlichen Gitterparametern $d_{1}$ und $d_{2}$ übereinanderliegen oder Kristallite mit gleichem Gitterparameter um einen kleinen Winkel $\beta$ gegeneinander verdreht sind. Die Streifenmuster haben die Periodizitätslängen von [Edington, 1975 / Alexander, 1997]: 


$$
D=\frac{d_{1} d_{2}}{d_{1}-d_{2}} \text { bzw. } D=\frac{d}{2 \sin (\beta / 2)} \approx \frac{d}{\beta}
$$

Im allgemeinen überlagern sich Gitter unterschiedlicher Gitterkonstanten, die schwach gegeneinander verdreht sind. Hier gilt [Hirsch, 1977 / Edington, 1975]:

$$
D \approx \frac{d_{1} d_{2}}{\left[\left(d_{1}-d_{2}\right)^{2}+d_{1} d_{2} \beta^{2}\right]^{1 / 2}}
$$

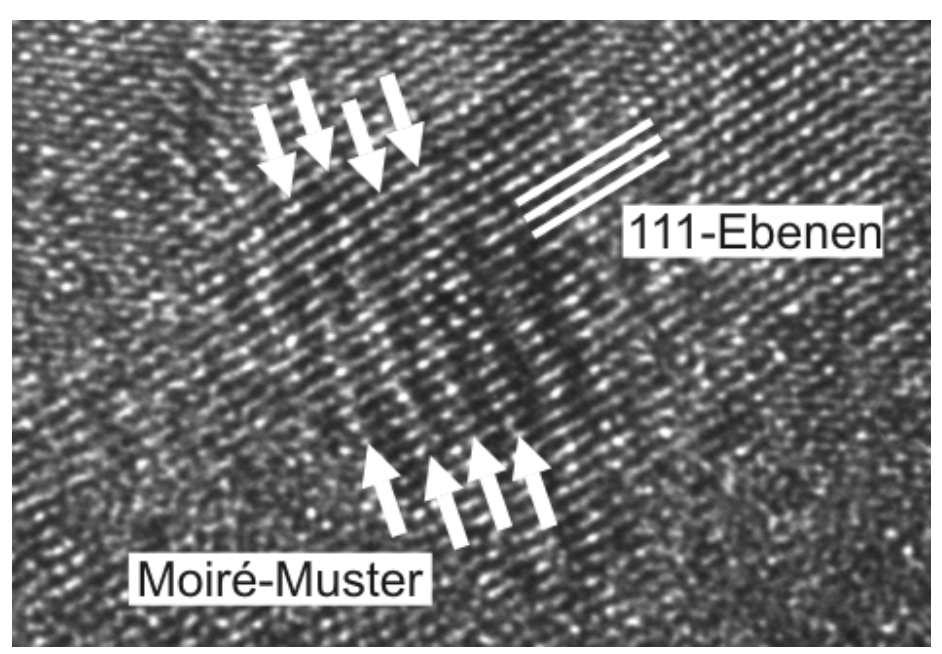

Abbildung 4.6: Netzebenenabbildung: Durch übereinanderliegende Körner unterschiedlicher Orientierung entsteht ein Moiré-Muster.

\subsection{TEM-Probenpräparation}

Um auf Substraten abgeschiedene Filme im TEM zu untersuchen, müssen die Proben bis auf eine Dicke von $<60 \mathrm{~nm}$ gedünnt werden, dass sie für die Elektronen durchstrahlbar sind. Je nach Ansicht (Querschnitt oder Aufsicht) ist eine bestimmte Probenpräparation erforderlich. Im folgenden werden die beiden typischen Präparationen für die transmissionselektronenmikroskopischen Untersuchungsmethoden beschrieben. Dies sind zum einen die Querschnittspräparation, bei der der Film im TEM von der Seite betrachtet wird (cross section), und zum anderen die Aufsichtspräparation (planar-oder plane view).

\section{- Aufsicht}

Für die Aufsichtsuntersuchungen wurde das Schichtsystem (Substrat-Film) einseitig von der Substratseite auf eine Dicke von ca. $80 \mu \mathrm{m}$ mechanisch (Schleifpapier, 800 - 4000er Kör- 
nung) gedünnt. Anschließend wurde mit einem Muldenschleifgerät (Dimple Grinder, Fa. Ga$\tan$ ) eine Mulde in die Probe gearbeitet, so dass die Probe an der dünnsten Stelle ca. 10 $20 \mu \mathrm{m}$ dick ist. Das Aufkleben eines Tantalringes dient zur mechanischen Stabilisation der Probe. Durch einseitigen Argon-Ionenbeschuss mit $5 \mathrm{kV}$ wird die Probe bis zur Lochentstehung vom Substrat her gedünnt. Zum Schluss wird die Probe mit Argon-Ionen beidseitig poliert (Ionenätze, Typ Duo Mill, Fa. Gatan).

Für die Untersuchung des Wachstums der ersten Nanometer Filmdicke, wurden mit Kohlenstoff beschichtete Kupfernetzchen (200 $\mu$ m Maschenweite, Fa. Plano) mit 30 - 60 nm YSZ beschichtet. Diese Proben sind ohne Präparation direkt im Elektronenmikroskop in Aufsicht durchstrahlbar.

\section{- Querschnitt}

Die Querschnittspräparation ist eine deutlich aufwändigere Präparation als die Aufsichtspräparation. Sie lässt sich in mehrere Schritte unterteilen. Der erste Schritt ist das Einbetten der Proben in einen Edelstahlzylinder (Abbildung 4.7). Die Probe wird zunächst in Streifen von 1,75 mm Breite gesägt. Diese werden anschließend mit Epoxy-Kleber (G2, Fa. Gatan) zusammengeklebt ("Film-auf-Film”). Dieses „Sandwich“ wird nun mechanisch mit Schleifpapier (800 - 2400er Körnung) beidseitig auf eine Dicke von ca. $300 \mu \mathrm{m}$ geschliffen. Der Streifen werden in den Schlitz eines Edelstahlvollzylinders $(\varnothing 3 \mathrm{~mm})$ eingeklebt, der wiederum in einen Edelstahlhohlzylinder geklebt wird. Die so eingebettete Probe wird mittels einer Innenlochsäge in Scheiben von ca. $1 \mathrm{~mm}$ Dicke gesägt.

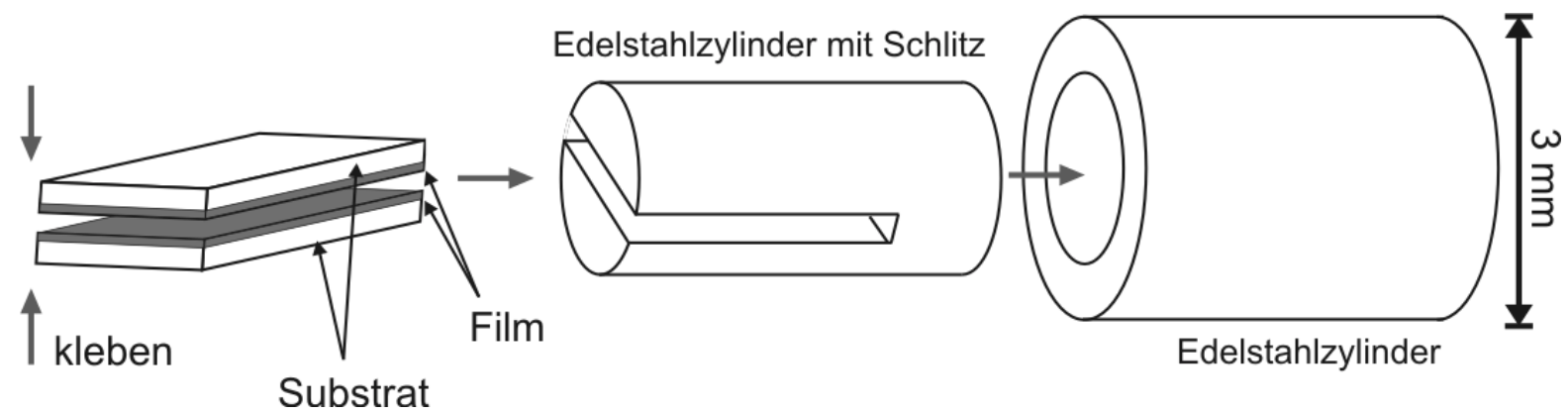

Abbildung 4.7: Querschnitts-Präparpation, 1.Teil: Das Einbetten des Schichtpakets in einen Edelstahlzylinder.

Im zweiten Schritt der Präparation wird eine Scheibe mit Schleifpapier (800 - 2400er Körnung) mechanisch von beiden Seiten mit Hilfe eines Handschleifgeräts (Disk Grinder, Fa. Gatan) auf eine Dicke von ca. $50 \mu \mathrm{m}$ gedünnt. Anschließend schleift man mit einem Muldenschleifgerät (Dimple Grinder, Fa. Gatan) eine Mulde in die Probe, die so tief ist, dass die 
dünnste Probenstelle $\leq 20 \mu \mathrm{m}$ dick ist. Das Gerät verfügt über ein Schichtdickenmessgerät, mit dem man die Dicke der Probe während des Schleifprozesses bestimmen kann (vgl. Abbildung 4.8).
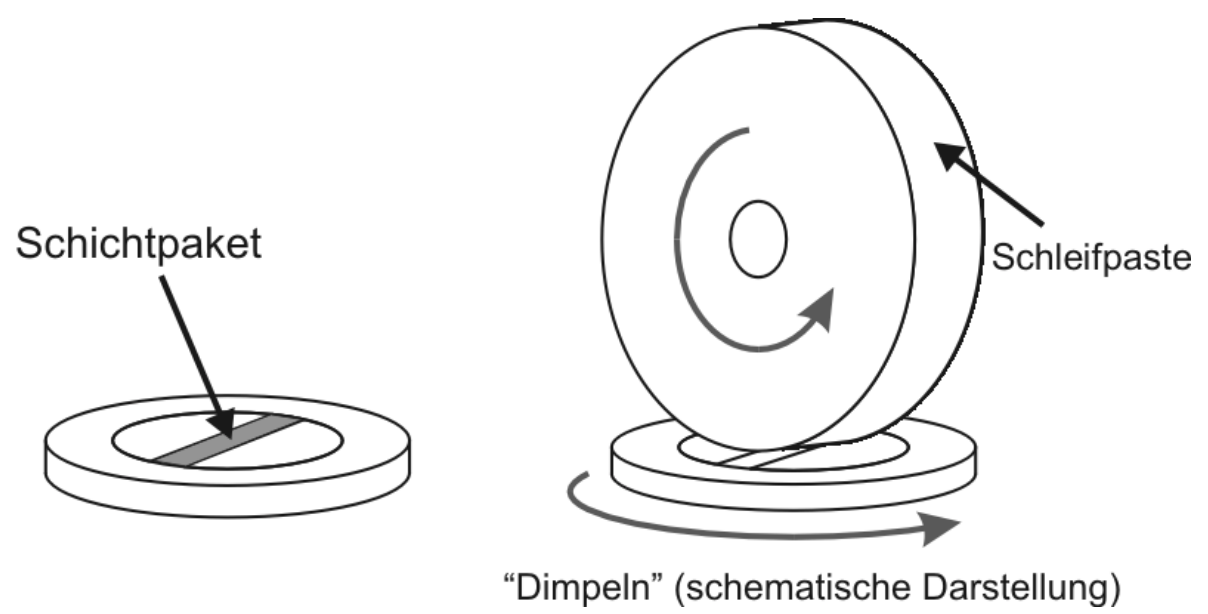

Abbildung 4.8: Querschnitts-Präparpation, 2. Teil: mechanisches Dünnen der Probenscheiben mittels Schleifpapier und Muldenschleifgerät (Dimpler)

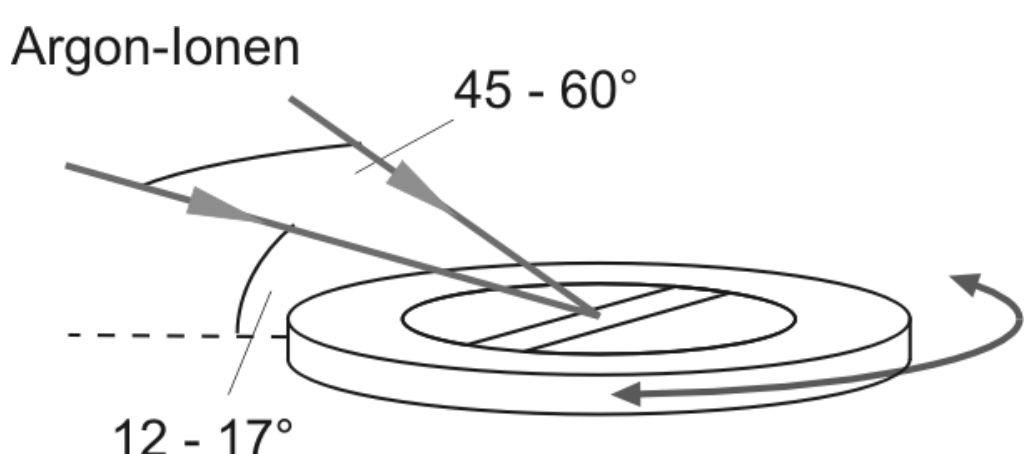

Abbildung 4.9: Querschnitts-Präparpation, 3. Teil: Die Probe wird durch Argon-Beschuss bis zur Lochentstehung gedünnt. Der Rand des Loches ist Elektronen-durchstrahlbar.

Im dritten und letzten Schritt wird die Probe mittels Argon-Ionen ( $5 \mathrm{kV}, 0,5 \mathrm{~mA})$ unter einem Winkel von $12-17^{\circ}$ bis zur Perforation gedünnt (Ionenätze, Typ Duo Mill, Fa. Gatan). Dabei ist es wichtig, dass der Ionenstrahl senkrecht zum Schichtpaket einfällt (vgl. Abbildung 4.9). Ein abschließendes Polieren mit Argon-Ionen ( $2 \mathrm{kV}, 0,5 \mathrm{~mA})$ beendet die Probenpräparation. Die Probe sollte nun am Lochrand eine Dicke von weniger als $60 \mathrm{~nm}$ haben, damit sie für die Hochauflösung mit Elektronen durchstrahlbar ist. 


\section{- Zur Definition der Blickrichtung bei Querschnittsaufnahmen}

Da durch den einfallenden Ionenstrahl eine Raumrichtung ausgezeichnet ist, sind anisotrope Strukturen denkbar. So wurden die Querschnittsproben sowohl senkrecht als auch parallel zur Projektion des unterstützenden Ionenstrahls auf die Substratoberfläche geschnitten. Es wird im folgenden zwischen der Blickrichtung ,parallel-“ und ,senkrecht zur Projektion des unterstützenden Ionenstrahls“ unterschieden. Abbildung 4.10 zeigt eine schematische Darstellung zur Definition dieser Blickrichtungen.

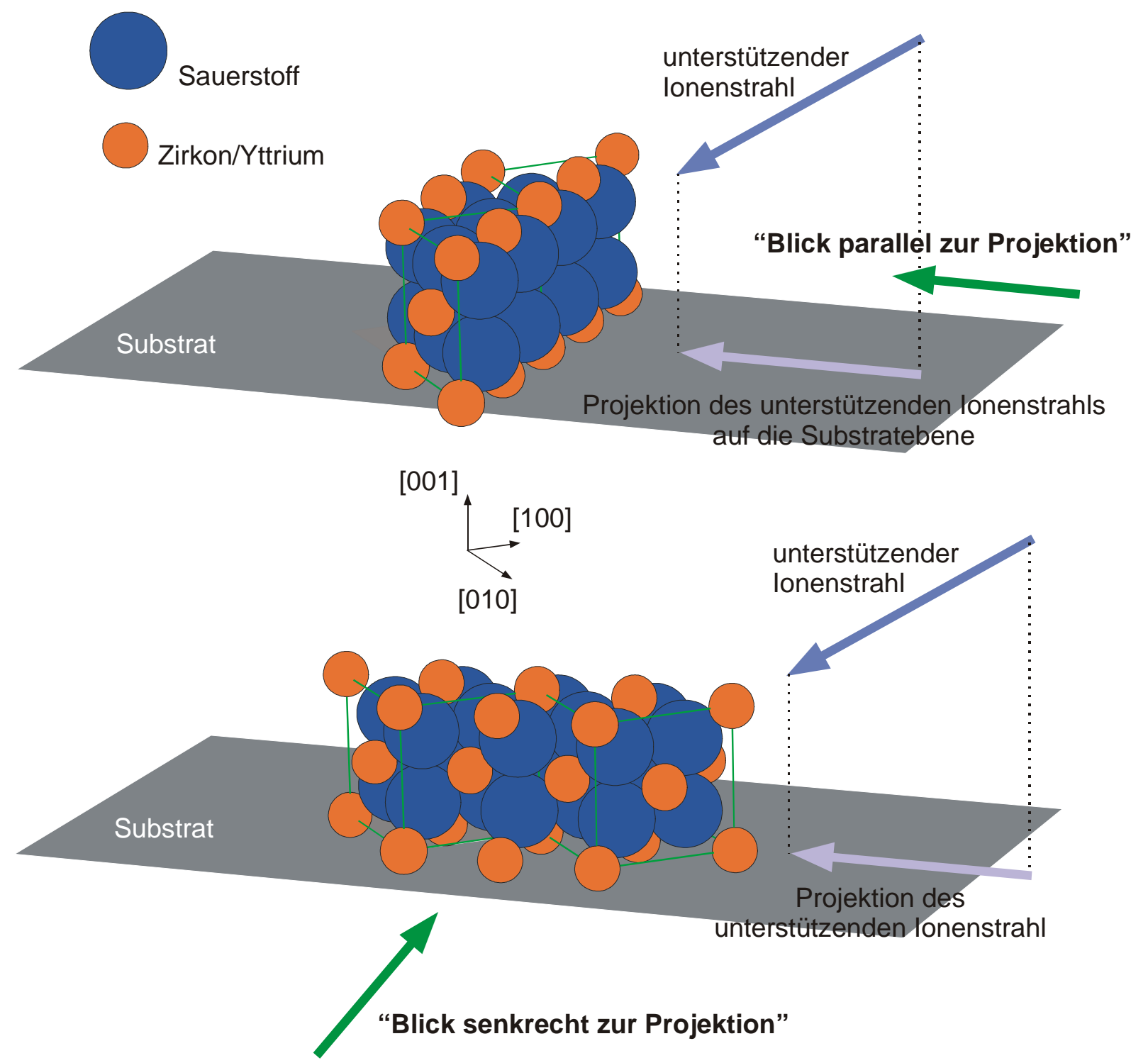

Abbildung 4.10: Schematische Darstellung zur Definition der Blickrichtungen bei Querschnittsaufnahmen. 


\section{Wachstum und Mikrostruktur von IBAD-YSZ- Schichten}

\subsection{Die Texturierung mittels IBAD}

Yu et al. zeigten erstmals, dass sich während der Deposition von Nb-Schichten mit einem zusätzlichen Ionenstrahl, der unter einem bestimmten Winkel auf das Substrat gerichtet ist, eine biaxiale Textur ausbildet [Yu et al., 1985]. Iijima und Mitarbeiter haben dieses Verfahren der ionenstrahlunterstützten Deposition (kurz: IBAD) erstmals angewendet um YSZ-Filme biaxial zu texturieren [Iijima et al., 1991]. Die Mechanismen, die zur Ausbildung einer Textur unter Ionenbeschuss führen, sind vom großen Interesse, aber nur in Teilaspekten verstanden.

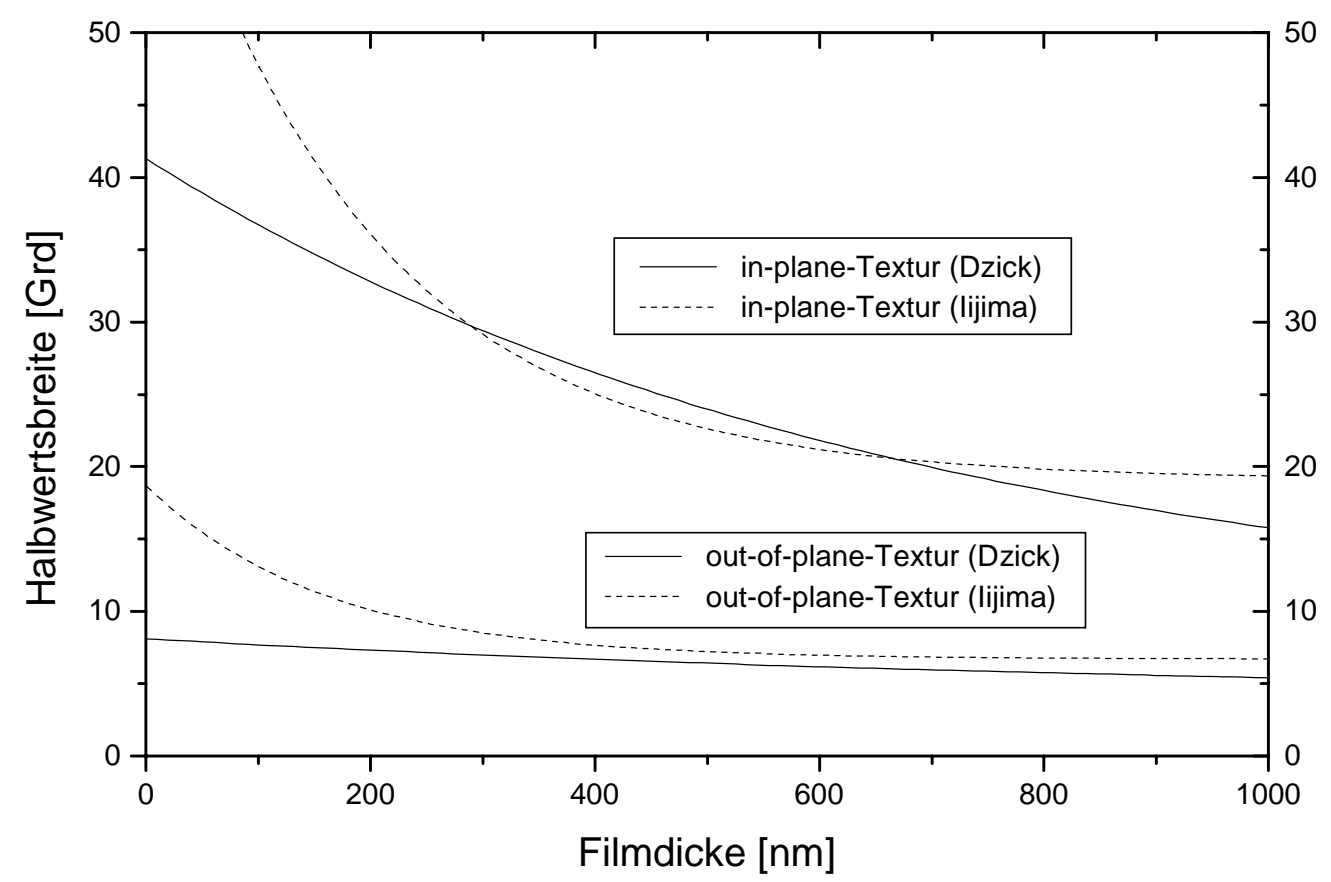

Abbildung 5.1: Entwicklung der Halbwertsbreite der in- und out-of-plane-Textur von IBADYSZ-Filmen gegen die Schichtdicke, nach [Iijima et al., 1998 / Dzick, 2000]. 
Die besten Texturen bei YSZ-Filmen werden bei Einsatz eines Argon-Ionenstrahls mit einer Ionenenergie von $250-350 \mathrm{eV}$ erreicht. Für eine ausgeprägte biaxiale Textur des YSZ muss der Einfallswinkel des Ionenstrahls $55^{\circ}$ zur Substratnormalen betragen (Winkel zwischen $<100>$ und $<111>$ ). Die verwendeten Kaufman-Ionenquellen generieren einen Ionenstrahl, dessen Partikel weitgehend parallele Trajektorie aufweisen, die Winkeldivergenz ist kleiner $7^{\circ}$ [Wiesmann, 1998].

Typische Röntgenmessung bei optimierten Bedingungen zeigen sowohl eine Verbesserung der in- als auch out-of-plane-Textur mit zunehmender Schichtdicke (vgl. Abbildung 5.1). Die Entwicklung der Halbwertsbreite lässt sich durch einen exponentiellen Abfall beschreiben, der sich asymptotisch einem Grenzwert nähert. In der Literatur wird dieser Verlauf durch ein Überwachsen fehlorientierter Körner beschrieben [Ying et al., 1996].

Neben der Texturverbesserung in IBAD-YSZ-Filmen mit zunehmender Schichtdicke zeigten weiterführende röntgenographische Untersuchungen ein Vorhandensein von (110)orientierten Körnern in den ersten Nanometern Filmdicke [Dzick, 2000]. Der Anteil dieser Körner nimmt mit zunehmender Schichtdicke ab, während sich die biaxiale Textur der (001)Körnern ausgeprägt. Dzick interpretiert das Vorhandensein der (110)-Drahttextur als spannungsindiziert, da durch diese die Spannungen in dem Film minimiert werden [Dzick, 2000].

Untersuchungen zum Einfluss des Substrates auf die Texturausbildung haben gezeigt, dass auf einkristallinen und texturierten Substraten epitaktische Effekte auftreten können. Der unterstützende Ionenstrahl kann offenbar die Epitaxietemperatur absenken. Auf YSZEinkristallen wächst die Anfangsschicht epitaktisch kristallin auf, ebenso auf texturierten YSZ- und $\mathrm{CeO}_{2}$-Filmen [Wiesmann, 1994 / Wiesmann, 1998]. Auf einer einmal gebildeten Initialschicht wächst der Film somit zunächst unter Beibehaltung der Initialtextur weiter. Dies verhindert z.B., dass auf polykristallinem YSZ hochtexturierte YSZ-Filme mittels IBAD abgeschieden werden können. Es ist daher wichtig, das Anfangsstadium des Filmwachstums genauer zu untersuchen.

Der Einfluss der Stromdichte des unterstützenden Ionenstrahls auf das Filmwachstum ist ebenfalls von besonderem Interesse. Neben den bereits angesprochenen Parametern, wie Ionenart und Energie der einfallenden Ionen, ist als weiterer Parameter das Verhältnis der Stromdichte von unterstützenden und deponierten Atomen, das sogenannte $\eta$-Verhältnis, wichtig. Wie schon in Abschnitt 3.1 erwähnt, ist $\eta$ definiert als:

$$
\eta=\frac{\text { Ionenauftreffrate }}{\text { Teilchendepositionsrate }}
$$


Typische Werte für die dimensionslose Größe $\eta$ bei der Sputterdeposition betragen $1-3,7$ [Wiesmann, 1998]. Die besten Texturierungsgrade werden bei bestimmten $\eta$-Werten erreicht, die von Material und der verwendeten Abscheidemethode abhängen. Daher wurden YSZFilme durch Variation der Stromdichte des unterstützenden Ionenstrahls mit unterschiedlichen $\eta$-Verhältnissen hergestellt und verglichen. Dabei lag das Hauptaugenmerk der Untersuchungen auf der Charakterisierung der nach Maßgabe der schnellstmöglichen Texturverbesserung optimierter hergestellter IBAD-YSZ-Filme. In Tabelle 3.1, Seite 9 sind die genauen Depositionsparameter angegeben.

Röntgenographische Untersuchungen, wie sie hauptsächlich zur Charakterisierung dünner Filme durchgeführt werden, beschreiben die Texturausbildung beim Wachstum ionenstrahlunterstützter YSZ-Schichten über die Veränderung von Orientierungsverteilungen. Die Messungen ergeben ein Mittel über große Filmbereiche sowohl lateral als auch in Wachstumsrichtung der Schicht. Bei der Fläche eines Röntgenstrahlfokusses von ca. $10^{6} \mu \mathrm{m}^{2}$ mittelt man so bei typischen Kornquerschnittsflächen der YSZ-Körner von $10^{-6}-10^{-4} \mu \mathrm{m}^{2}$ über $10^{10}-10^{12}$ Körnern. Über die Entwicklung der kristallographischen Ausrichtung und Mikrostruktur einzelner Körner sind damit keine Aussagen möglich. Da der Röntgenstrahl den gesamten Film durchdringt, können auch keine Aussagen gemacht werden, ob lokale Prozesse z.B. stetig (Überwachsen von Körnern) oder diskontinuierlich (z.B. Keimbildung anders orientierter Körner) ablaufen.

Um Aussagen über Orientierungen einzelner Körner und somit über die lokale Texturentwicklung machen zu können, ist die Transmissionselektronenmikroskopie (TEM) ein wichtiges Hilfsmittel. Durch die hohe Auflösung können detaillierte Analysen der lokalen Entwicklung durchgeführt werden, die eine Entscheidung über die Richtigkeit von auf makroskopischen Modellen beruhenden Theorien zulässt. So wurden IBAD-YSZ-Filme sowohl im Querschnitt als auch in Aufsicht untersucht. Mittels Beugungs- und Verkippungsexperimente lassen sich Aussagen über die Orientierungen einzelner Regionen treffen. Mit Hilfe der Hochauflösenden Transmissionselektronenmikroskopie (HREM) und der schnellen FourierTransformation (FFT) ist es zusätzlich möglich Orientierungen kristalliner Bereiche mit Durchmesser $\geq 5 \mathrm{~nm}$ zu bestimmen und so detaillierte Aussagen über die Texturentstehung und -entwicklung zu treffen. 


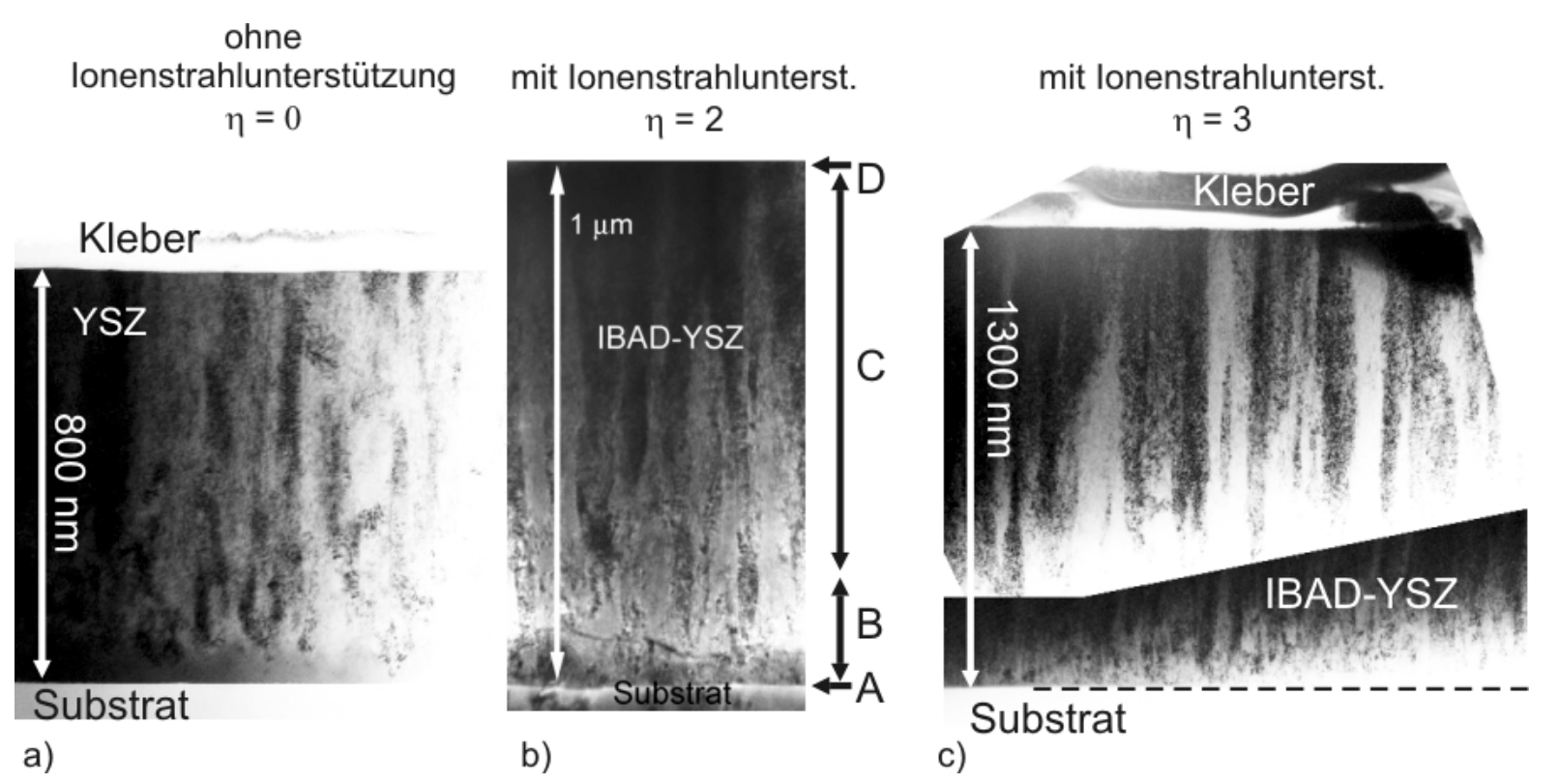

Abbildung 5.2: TEM-Querschnitts-Übersichtsaufnahmen von YSZ-Filmen, die ohne (a) und mit Ionenstrahlunterstützung $(b-c)$ hergestellt wurden.

Typische TEM-Übersichtsaufnahmen von YSZ-Filmen im Querschnitt, die bei verschiedenen $\eta$-Verhältnissen hergestellt wurden, sind in Abbildung 5.2 dargestellt. Die unterschiedlichen Graustufen in den Bildern entstehen durch unterschiedliche Orientierungen kristalliner Bereiche zum Elektronenstrahl. In allen untersuchten Filmen weisen die Hell-DunkelKontraste (wie in Bereich C, Abbildung 5.2 b) auf ein ausgeprägtes kolumnares Wachstum hin. Wie in Abbildung $5.2 \mathrm{zu}$ erkennen, haben die einzelnen Säulen ihren Ursprung offenbar nicht direkt am Substrat. Dieser Bereich zwischen Säulenursprung und Substrat ist für die Entstehung der gewünschten $(001)<100>$-Textur von großer Bedeutung (Bereich B in Abbildung 5.2 b). Er lässt sich in ein Frühstadium und Entstehung der (001)-Textur unterteilen. So sollen HREM- und FFT-Untersuchungen Aufschluss über die Eigenschaften des Filmwachstums in den ersten Nanometern Schichtdicke geben. Substratbeschaffenheit, Epitaxie und Eigenschaften der Keimschicht sind dabei von besonderem Interesse (Bereich A in Abbildung $5.2 \mathrm{~b}$ ).

Im folgenden werden zunächst die röntgenographischen Untersuchungen zu den betrachteten YSZ-Filmen vorgestellt. Es folgen dann TEM-Untersuchungen zu den Frühstadien des Wachstums bzw. zur Ausbildung der (001)-Textur. 


\subsection{Röntgenographische Untersuchungen}

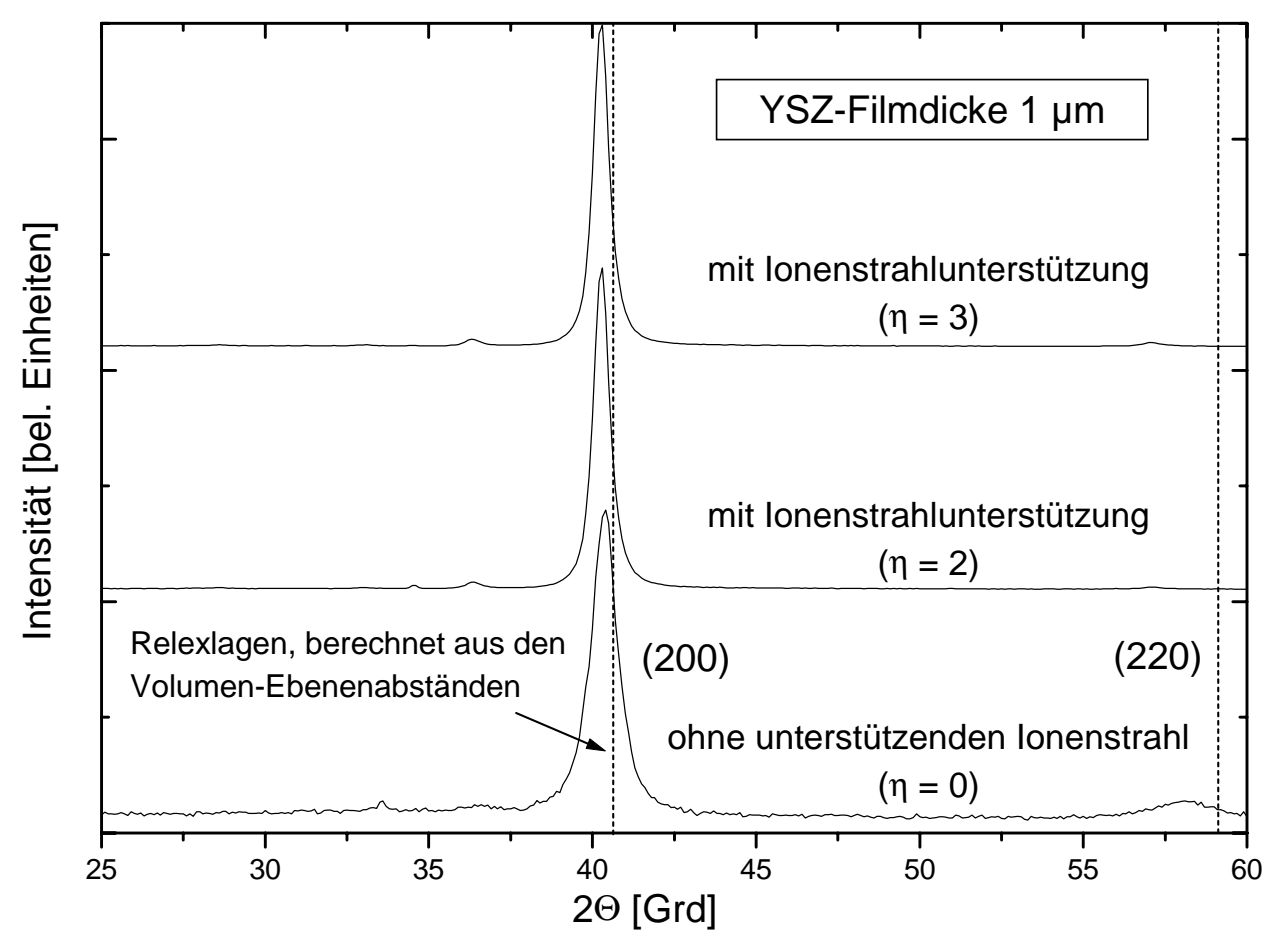

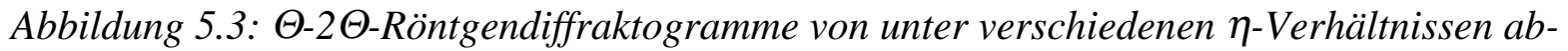
geschiedenen YSZ-Filmen. Die senkrecht gestrichelten Linien geben die berechneten Reflexlagen vom YSZ-Einkristall an. Die Diffraktogramme sind auf die maximale Intensität normiert.

In Abbildung 5.3 sind $\Theta-2 \Theta$-Röntgendiffraktogramme von bei verschiedenen $\eta$-Verhältnissen abgeschiedenen YSZ-Filme zu sehen. Die Dicke der Filme beträgt ca. $1 \mu \mathrm{m}$. Die Filme weisen unabhängig vom $\eta$-Verhältnis praktisch eine reine (001)-out-of-plane-Textur auf. Nur beim ohne Ionenstrahlunterstützung $(\eta=0)$ abgeschiedenen YSZ-Film ist noch ein (110)-Anteil erkennbar. Vergleicht man diese Messungen mit Röntgenographischen Messungen an ca. $300 \mathrm{~nm}$ dicken YSZ-Filmen (nicht gezeigt), so zeigen diese einen höheren Anteil an (110)-orientierten Bereichen. Der relative (110)-Anteil nimmt also für alle drei $\eta$-Werte mit zunehmender Schichtdicke ab. 


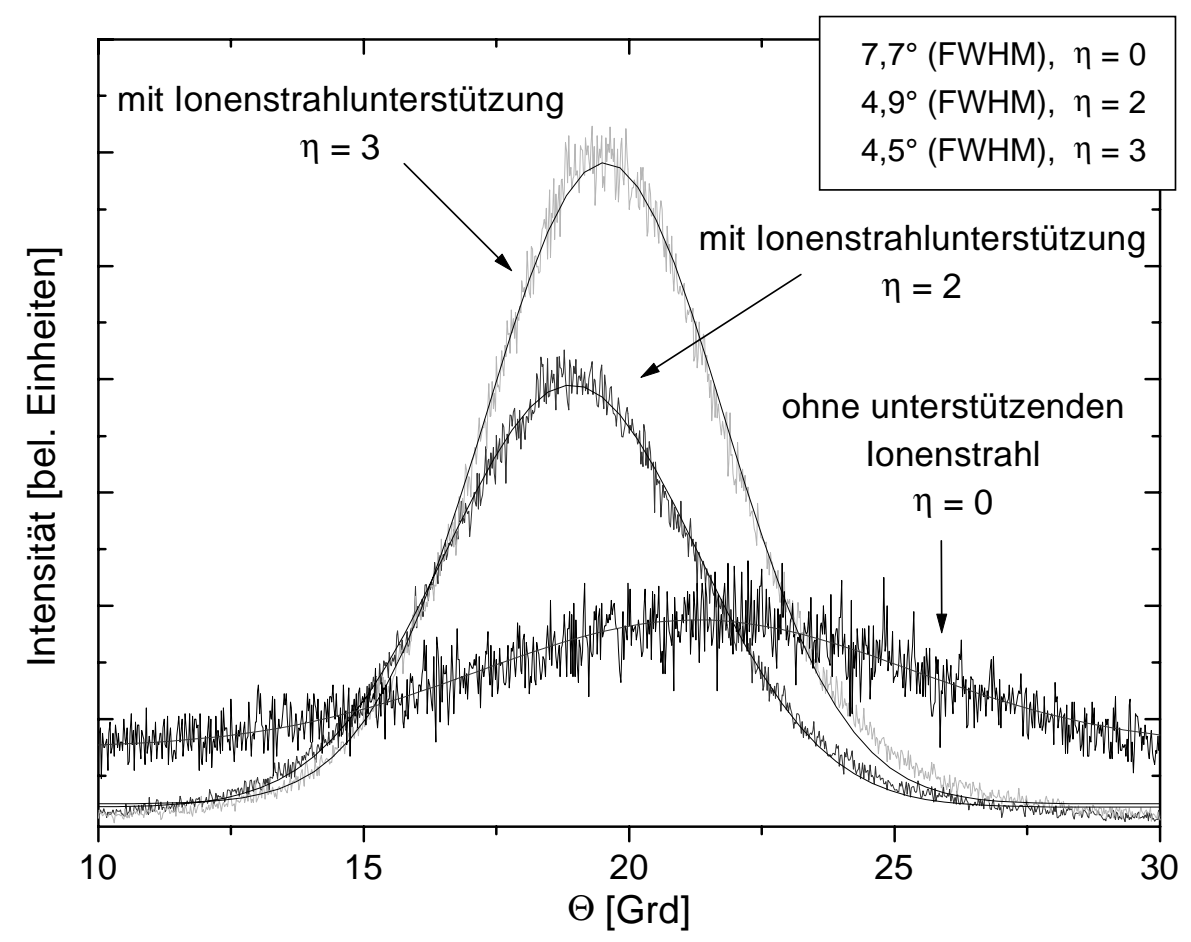

Abbildung 5.4: Rockingkurven der (002)-Reflexe von bei verschiedenen $\eta$-Verhältnissen abgeschiedenen YSZ-Filmen, Dicke der Filme ca. $300 \mathrm{~nm}$. Die Messungen erfolgten parallel zum unterstützenden Ionenstrahl. Die Halbwertsbreiten (FWHM) der jeweiligen Kurve sind oben angegeben. Die Intensität der Rockingkurve des mit $\eta=0$ abgeschiedenen Films wurde zur besseren Darstellung verfüffacht. Die Verschiebung der Maxima beruht auf einer leichten Verkippung der Probe bei der Messung.

Die out-of-plane-Textur lässt sich anhand der Halbwertsbreite (FWHM) der Rockingkurve angeben. Abbildung 5.4 zeigt die Rockingkurven von ca. $300 \mathrm{~nm}$ dicken YSZ-Filmen, die bei verschiedenen $\eta$-Verhältnissen hergestellt wurden. Die berechneten Halbwertsbreiten (FWHM) sind in Tabelle 5.1 aufgeführt. Die Rockingkurven wurden jeweils parallel- und senkrecht zum einfallenden unterstützenden Ionenstrahl gemessen. Alle durchgeführte Röntgenmessungen deuten darauf hin, dass die out-of-plane-Textur der IBAD-YSZ-Filme in Richtung des unterstützenden Ionenstrahls geringfügig besser ist. Die out-of-plane-Textur des YSZ-Films, der mit $\eta=0$ abgeschieden wurde, verbessert sich mit zunehmender Filmdicke nicht. Dagegen zeigen die YSZ-Filme, die mit Ionenstrahlunterstützung hergestellt wurden, eine deutliche Verbesserung. 


\begin{tabular}{|c|c|c|c|}
\hline & & \multicolumn{2}{|c|}{ Messrichtung } \\
\hline & & parallel & senkrecht \\
\hline & $\eta$-Verhältnis & FWHW [Grd] & FWHW [Grd] \\
\hline \multirow[t]{3}{*}{ Filmdicke: $300 \mathrm{~nm}$} & 0 & 7,7 & 8,4 \\
\hline & 2 & 4,9 & 5,5 \\
\hline & 3 & 4,5 & 5,25 \\
\hline \multirow[t]{3}{*}{ Filmdicke: $1000 \mathrm{~nm}$} & 0 & 7,9 & 10,9 \\
\hline & 2 & 3,8 & 4,1 \\
\hline & 3 & 3,8 & 4,3 \\
\hline
\end{tabular}

Tabelle 5.1: Halbwertsbreiten (FWHM) der gemessenen Rockingkurven von unter verschiedenen $\eta$-Verhältnissen abgeschiedenen YSZ-Filmen unterschiedlicher Dicke. Die Messungen erfolgten parallel- und senkrecht zum unterstützenden Ionenstrahl.

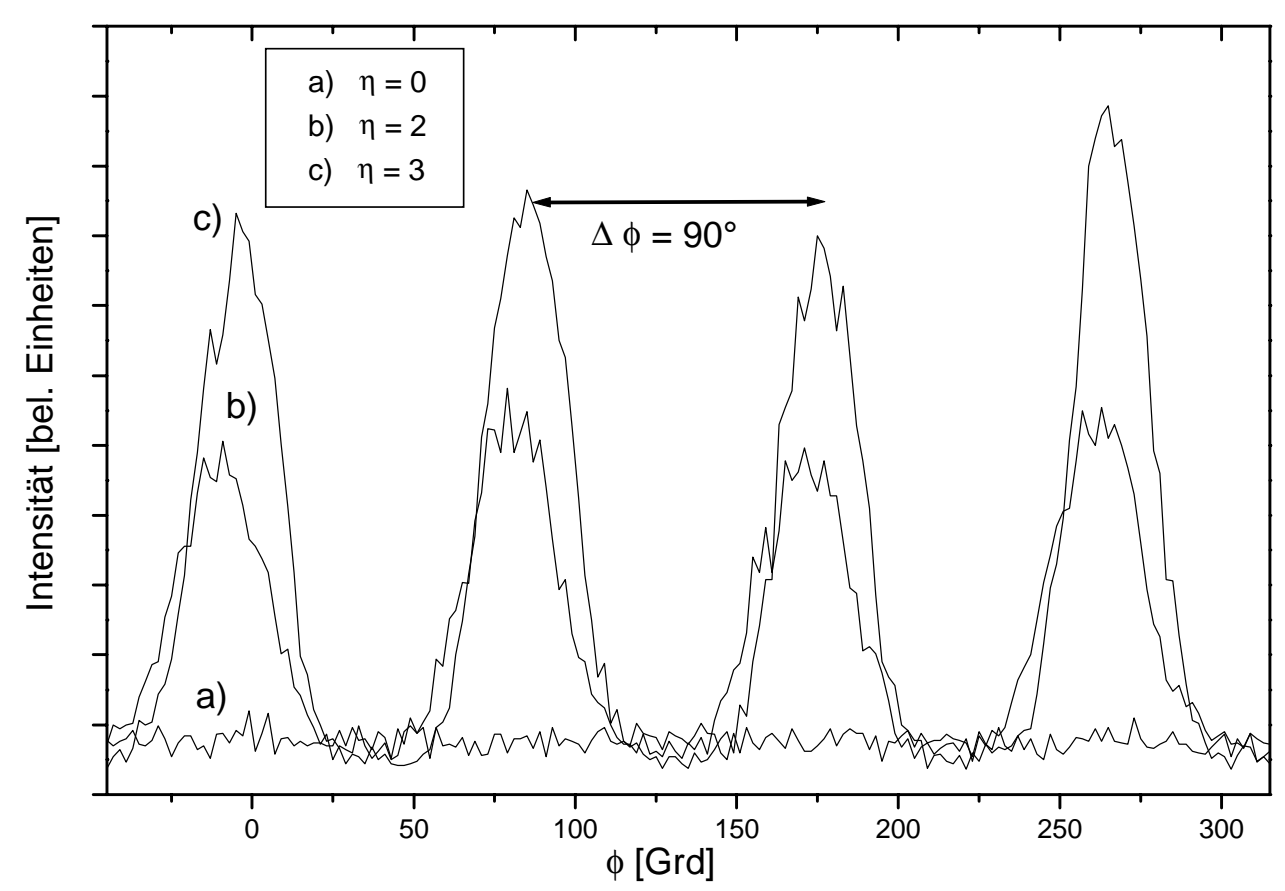

Abbildung 5.5: $\phi$-Scans der \{111\}-Reflexe von ca. $300 \mathrm{~nm}$ dicken YSZ-Filmen, die bei unterschiedlichen $\eta$-Verhältnissen abgeschieden wurden. 
Während die Halbwertsbreite der Rockingkurve Aufschluss über die out-of-plane-Textur gibt, gibt die Halbwertsbreite des $\phi$-Scans die in-plane-Textur an. Abbildung 5.5 zeigt $\phi$-Scans der $\{111\}$-Reflexe von ca. $300 \mathrm{~nm}$ dicken YSZ-Filmen, die bei unterschiedlichen $\eta$-Verhältnissen abgeschieden wurden. Das Vorhandensein der vier Reflexe im Abstand von $90^{\circ}$ zeigt auf eine in-plane-Textur hin. In Tabelle 5.2 sind die aus den in den $\phi$-Scans auftretenden vier Reflexen gemittelten Halbwertsbreiten angeben. Während die YSZ-Filme, die ohne unterstützenden Ionenstrahl abgeschieden wurden, keine in-plane-Textur aufweisen, verbessert sich die in-plane-Textur der IBAD-YSZ-Filme mit zunehmender Filmdicke deutlich.

\begin{tabular}{|c|c|c|}
\hline YSZ-Filmdicke & $300 \mathrm{~nm}$ & $1000 \mathrm{~nm}$ \\
\hline ohne Ionenstrahlunterst. $(\eta=0)$ & Halbwertsbreiten aus $\phi$-Scan [Grd] \\
\hline mit Ionenstrahlunterst. $(\eta=2)$ & - & - \\
\hline mit Ionenstrahlunterst. $(\eta=3)$ & 23,9 & 16,0 \\
\hline
\end{tabular}

Tabelle 5.2: Gemittelte Halbwertsbreiten (FWHM) der vier $\{111\}$-Reflexe aus den gemessenen $\phi$-Scans, der unter verschiedenen Bedingungen abgeschiedenen YSZ-Filmen unterschiedlicher Dicke.

Zusammengefasst zeigen alle untersuchten YSZ-Filme eine ausgeprägte (001)-Textur, die mit einer Verringerung des (110)-Anteils mit zunehmender Filmdicke einhergeht. Diese Reduktion wird auch in YSZ-Filmen, die ohne Ionenstrahlunterstützung abgeschieden wurden, beobachtet. Der deutliche Unterschied der (110)-Anteile zwischen $\eta=0$ und $\eta \geq 2$, zeigt, dass der Ionenstrahl das Wachstum der (110)-orientierten Bereiche unterdrückt. Die primäre Wirkung des Ionenstrahls liegt aber in der systematischen Verbesserung der in- und der out-ofplane-Textur mit zunehmender Filmdicke.

\subsection{Frühstadien des Wachstums}

\subsubsection{Die Substratwelligkeit}

Vor jeder Deposition werden alle Substrate mittels des unterstützenden Ionenstrahls $\left(400 \mathrm{~V}, J \geq 300 \mu \mathrm{A} / \mathrm{cm}^{2}, 55^{\circ}\right) 2-3$ Minuten geätzt, um Lösungsmittelrückstände und Absorbate an der Oberfläche zu beseitigen. Danach beginnt sogleich die Deposition. Die Haftung der Filme auf dem gereinigten Substrat wird so deutlich verbessert [Dzick, 2000]. Durch das 
Ätzen wird die Substratoberfläche verändert. Das Abtragen und Verlagern von Atomen durch den Ionenbeschuss bewirkt eine Neustrukturierung der Oberfläche. Es bildet sich eine periodische wellenförmige Struktur aus, die in der Literatur als „Ripples“ bezeichnet wird [Navez et al., 1962]. Diese Struktur wurde auch von Wiesmann an IBAD-YSZ-Filmen beobachtet [Wiesmann, 1998].

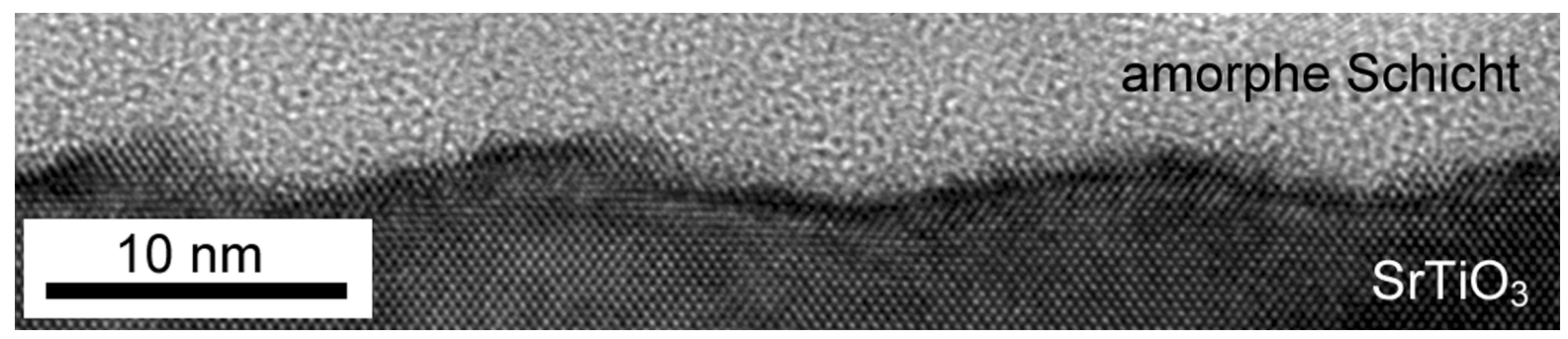

a) $\mathrm{SrTiO}_{3}$-Oberfläche im Querschnitt,

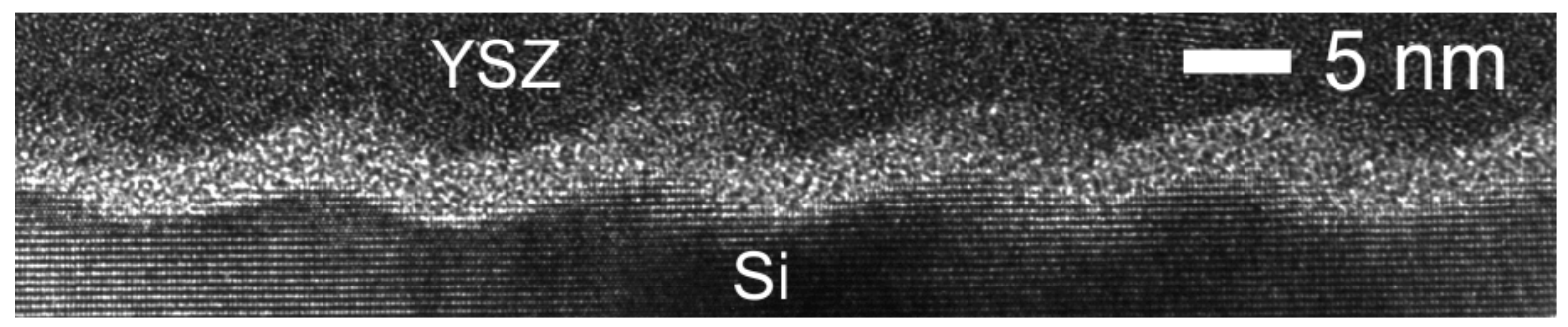

b) Wellenstruktur der Silizium-Oberfläche

Abbildung 5.6: Wellenstruktur auf verschiedenen Substratoberflächen, HREMQuerschnittsaufnahmen; Man blickt senkrecht zur Projektion des unterstützenden Ionenstrahls. Die Substrate wurden vor der Deposition mittels des unterstützenden Ionenstrahls einige Minuten gereinigt.

In Abbildung 5.6 sind hochaufgelöste TEM-Bilder zu sehen, die verschiedene Substrate im Querschnitt zeigen. Man blickt in allen Aufnahmen senkrecht zur Projektion des unterstützenden Ionenstrahls. Die Substrate waren vor dem Ätzen glatt. Deutlich ist eine Wellenstruktur an der Substratoberfläche zu erkennen. Die Wellenlänge der Ripples liegt zwischen 8 und $14 \mathrm{~nm}$. Die Amplitude beträgt ca. 2,5 nm. Es wurden keine deutlichen Unterschiede bei verschiedenen Substraten festgestellt.

Alle Silizium-Substrate zeigen zusätzlich eine $3-5 \mathrm{~nm}$ dicke homogene Siliziumoxidschicht. Da während des Ionenätzens der Depositionsvorgang gestartet wird, ist es möglich, dass diese $\mathrm{SiO}_{2}$-Schicht direkt durch Oxidation der gereinigten Siliziumoberfläche entsteht. Ebenfalls scheint es möglich zu sein, dass durch eine Diffusion des Sauerstoffs durch das aufwachsende YSZ eine nachträgliche Oxidation der Siliziumoberfläche stattfindet. Zum Vergleich haben Silizium-(001)-Flächen bei Raumtemperatur natürliche Oxidschichten von 
1,7 nm Dicke [Behner et al., 1992]. Möglicherweise führt der Ionenbeschuss zu einer gestörten Oberflächenstruktur, die dazu führt, dass der Sauerstoff tiefer als bei idealen Oberflächen ins Volumen eindringen kann, bevor sich eine passivierte Oxidschicht ausbildet, bzw. die Oxidationskinetik durch das aufwachsende YSZ eingeschränkt wird.

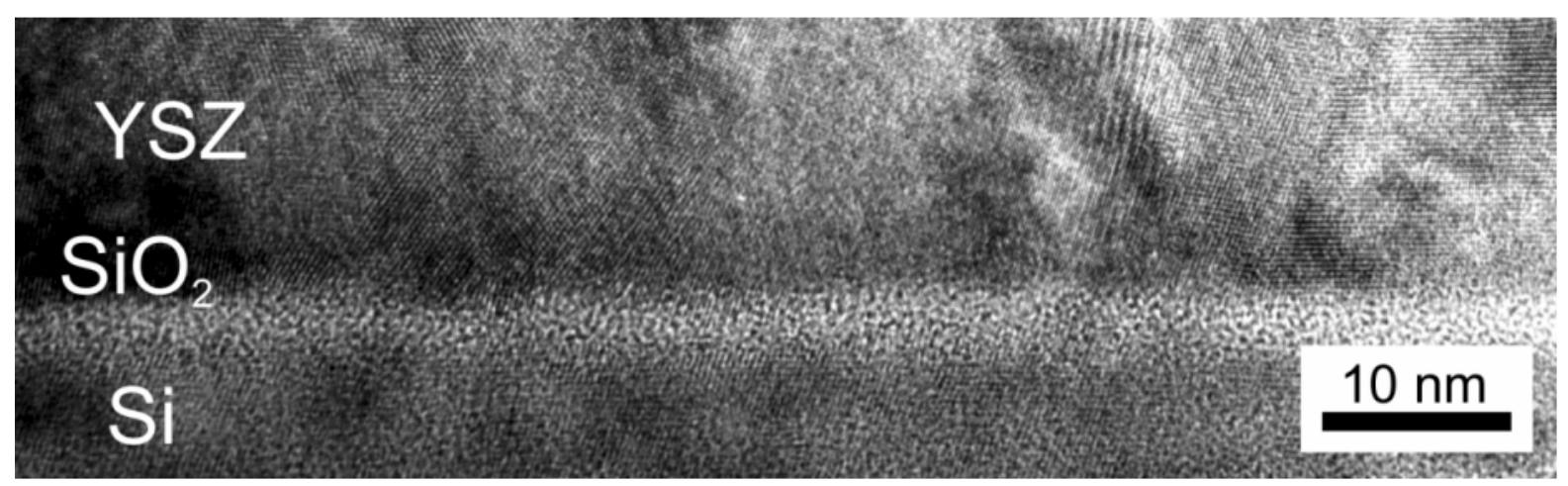

Abbildung 5.7: HREM-Querschnittsaufnahme eines Siliziumsubstrates, man blick parallel zur Wellenfront bzw. Projektion des unterstützenden Ionenstrahls (vgl. Abbildung 5.6).

Die Wellenfronten verlaufen senkrecht zum unterstützenden Ionenstrahl, das zeigen TEMUntersuchungen an Proben, in denen man parallel zur Projektion des unterstützenden Ionenstrahls blickt, wie z.B. in Abbildung $5.7 \mathrm{zu}$ sehen ist. In dieser Blickrichtung ist eine nicht gewellte Substratoberfläche zu erkennen.

Die Wellenstruktur zeigt einen überwiegend asymmetrischen Charakter. Selten sind symmetrischen Wellen zu beobachten. Abbildung 5.9 zeigt als Beispiel einer asymmetrischen Welle einen Bereich der (111)-Silizium-Oberfläche. Für diese Aufnahme wurde das SiliziumSubstrat mit der <211>-Richung parallel zum Elektronenstrahl orientiert, dies entspricht einem Blick senkrecht zur Projektion des Unterstützenden Ionenstrahls. An der steilen, zum unterstützenden Ionenstrahl gerichteten Flanke wird ein Winkelbereich von $30^{\circ}$ bis $35^{\circ}$ zur Substratnormalen beobachtet. Für (111)-orientiertes Silizium, dessen $<211>$-Richtung parallel zur Wellenfront verläuft bildet die entstehende Oberfläche somit keine niedrig indizierte Ebene, insbesondere liegt somit auch keine niedrig indizierte Ebene zum unterstützenden Ionenstrahl (Einfallswinkel: $55^{\circ}$ ) gerichtet. Somit bleibt unklar, ob die asymmetrische Struktur aufgrund von orientierungsabhängigen Ätzraten entsteht.

\subsubsection{Der Einfluss der Substratwelligkeit auf das Wachstum}

Die oben beschriebenen Substratoberfläche stellt die effektive Oberfläche für die YSZBeschichtung dar. Auf allen nicht-epitaxiefähigen Substraten zeigt sich in den weiteren Wachstumsstadien nach Maßgabe der röntgenographischen Untersuchungen die gleiche Tex- 
turentwicklung. Exemplarisch für nicht-epitaxiefähige Unterlagen wurden $\mathrm{Si}$ bzw. $\mathrm{SiO}_{2}$, amorphes $\mathrm{Al}_{2} \mathrm{O}_{3}$ und mit Kohlenstoff bedampfte Kupfernetzchen als Substrat untersucht. Die Stromdichte des unterstützenden Ionenstrahls wurde so gewählt, das $\eta=2$ ist (vgl. Tabelle 3.1, Seite 9).

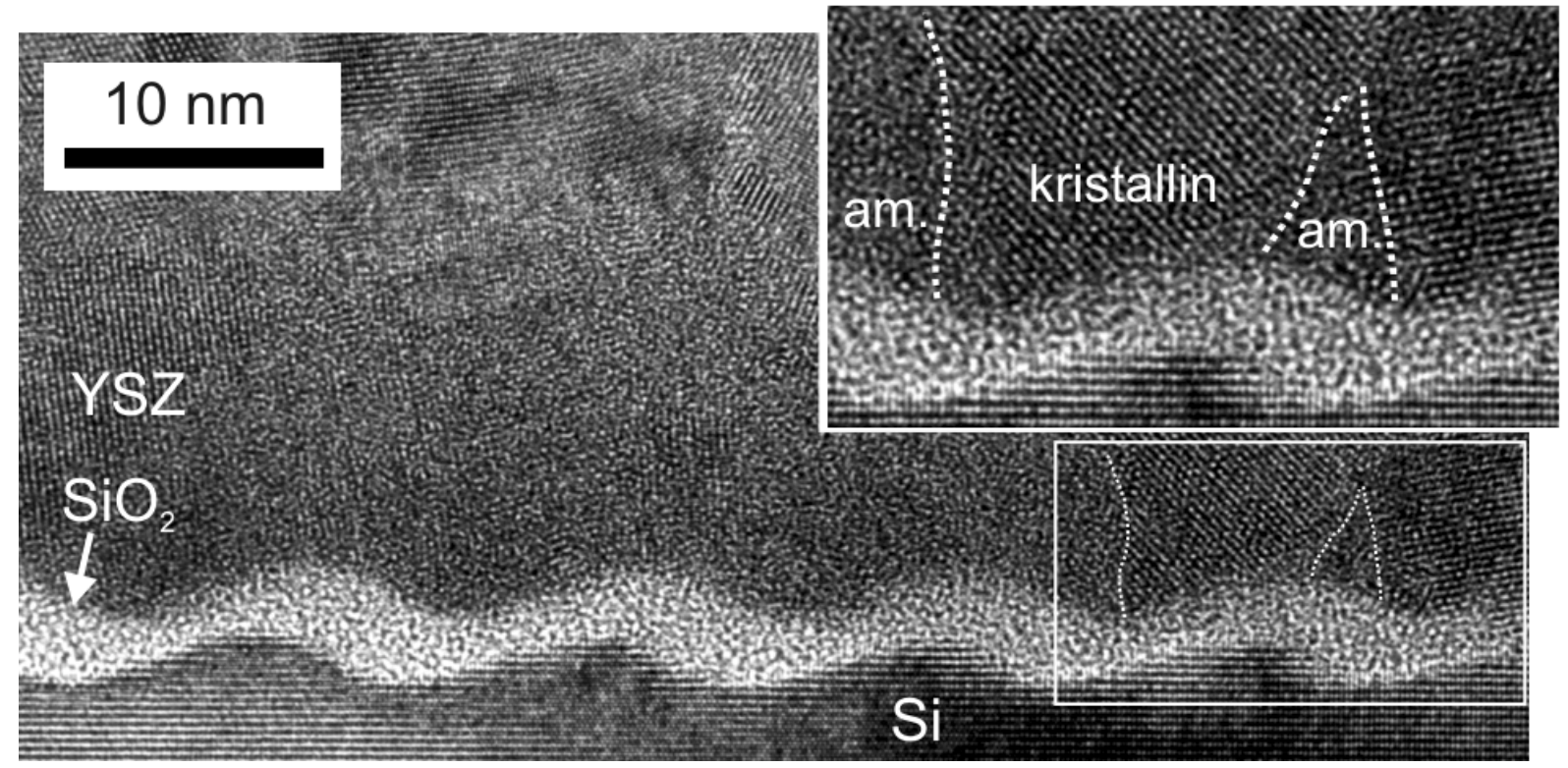

Abbildung 5.8: HREM-Querschnittsaufnahme von IBAD-YSZ, das bei $\eta=2$ abgeschieden wurde: Die ersten ca. $10 \mathrm{~nm}$ IBAD-YSZ-Filmdicke zeigen überwiegend amorphen Charakter. Keime an der Substratoberfläche bilden sich bevorzugt an den flachen Flanken der Ripples (s. Vergrößerung).

Abbildung 5.8 zeigt eine hochaufgelöste TEM-Querschnittsaufnahme der ersten Nanometer des YSZ-Films. Man blickt entlang der Wellenfront des Substrats, dies entspricht einer Sicht senkrecht zur Projektion des unterstützenden Ionenstrahls. In einer bis zu $10 \mathrm{~nm}$ dicken Filmschicht existieren abwechselnd Bereiche, die amorphen oder kristallinen Charakter zeigen (vgl. auch Abbildung 5.6). Der Anteil der amorphen Schichtbereiche beträgt ca. 80\%. Die laterale Größe der Körner direkt an der Substratoberfläche variiert stark und kann bis zu 10 nm betragen.

Um das Auftreten einer Anfangstextur (Textur-Offset) zu untersuchen wurden Beugungsexperimente in Aufsicht an mit YSZ beschichteten (Dicke ca. $30 \mathrm{~nm}$ ) Kohlenstofffilmen durchgeführt. Aus der Analyse ergibt sich, dass die Körner, die an der Substratoberfläche ankeimen keine Vorzugsorientierungen aufweisen (s. auch Abbildung 5.12 b, Seite 32). So besteht auch keine Epitaxierelation zwischen Substrat und YSZ-Film. Kristallite, die direkt an der $\mathrm{SiO}_{2}$-Substratoberfläche wachsen, haben ihren Ursprung oft an der flachen Flanke der 
asymmetrischen Welle. Abbildung 5.9 zeigt dieses Verhalten in einer hochaufgelösten TEMAufnahme. Um die Asymmetrie der Wellen zu zeigen, wurde in dem Bild die Substratoberseite hervorgehoben. An der flachen Flanke der Welle kristallisiert ein Korn, wogegen über der steilen Flanke ein amorpher Bereich zu erkennen ist. Die dazugehörende Fast-FourierTransformation (FFT) zeigt das typische Muster von Bereichen, die im Realbild keine kristalline, d.h. periodische Struktur zeigen.

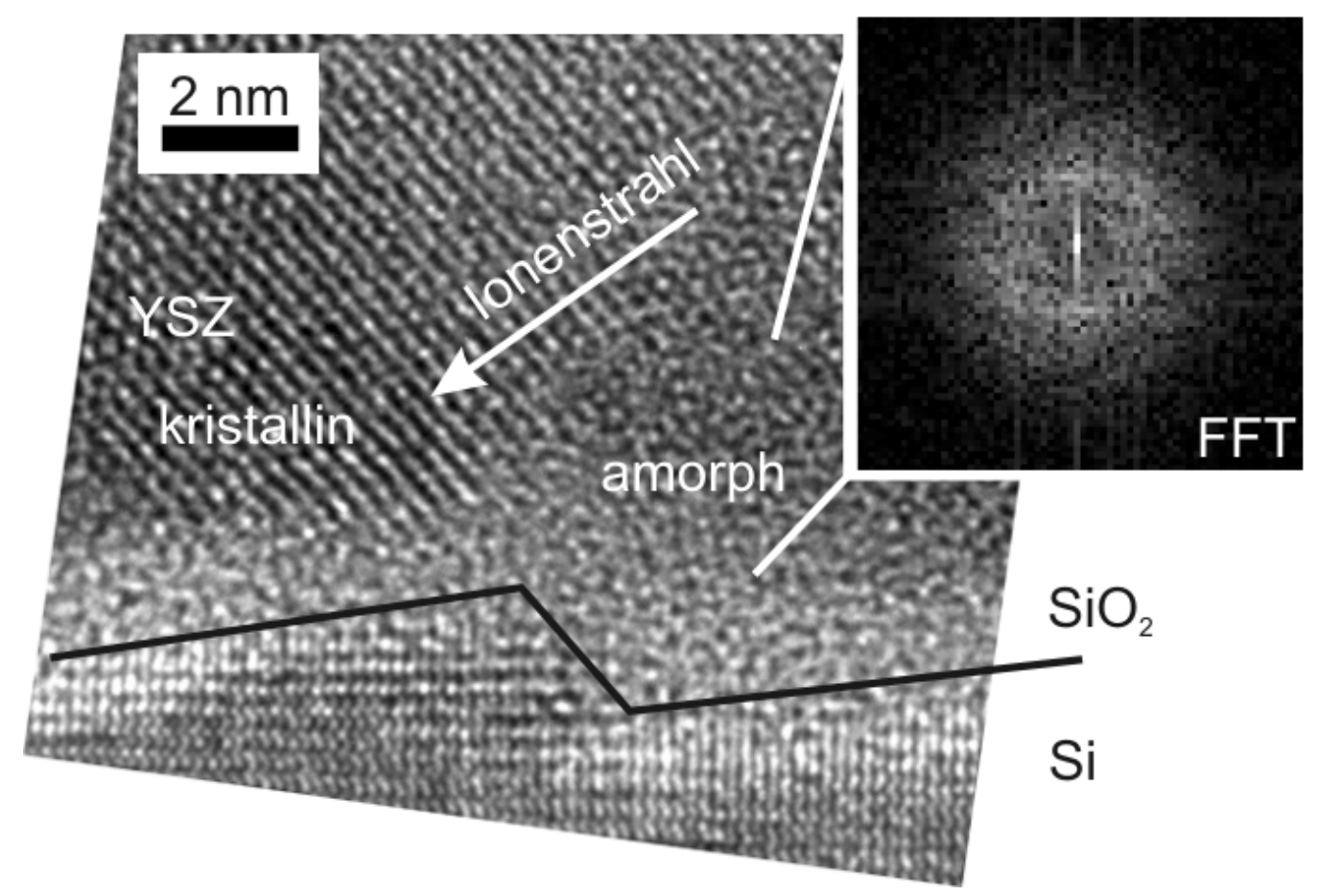

Abbildung 5.9: HREM-Querschnittsaufnahme, Blick parallel zur Wellenfront. Die FastFourier-Transformation (FFT) zeigt das typische Muster eines nichtkristallinen Bereiches, wie sie in den ersten $10 \mathrm{~nm}$ Schichtdicke häufig vorkommen. Körner in diesem Bereich keimen bevorzugt an den flachen Wellenflanken an.

Demgegenüber variiert die Ausdehnung der Kristallite entlang der Wellenfront stark und kann bis zu $20 \mathrm{~nm}$ betragen. Abbildung 5.10 zeigt eine Querschnittsaufnahme, bei der die Probe senkrecht zur Wellenfront gedünnt wurde. Man blickt parallel zur Projektion des unterstützenden Ionenstrahls. In der Mitte der Aufnahme ist ein kristalliner Bereich zu sehen, der direkt an der Siliziumoxidschicht entstanden ist. Der als „a“ gekennzeichnete Bereich zeigt ein Moiré-Muster. Diese Muster sind typischerweise in den ersten $200 \mathrm{~nm}$ Schichtdicke zu beobachten. Die abgeschätzten Verdrehungswinkel betragen wenige Grad. Bei Annahme der Probendicke von $30-60 \mathrm{~nm}$ zeigt sich, dass viele leicht gegeneinander verdrehte Körner in diesem Bereich übereinanderliegen. Die laterale Ausdehnung der Körner liegt in diesem Bereich in der Größenordnung der Probendicke. 


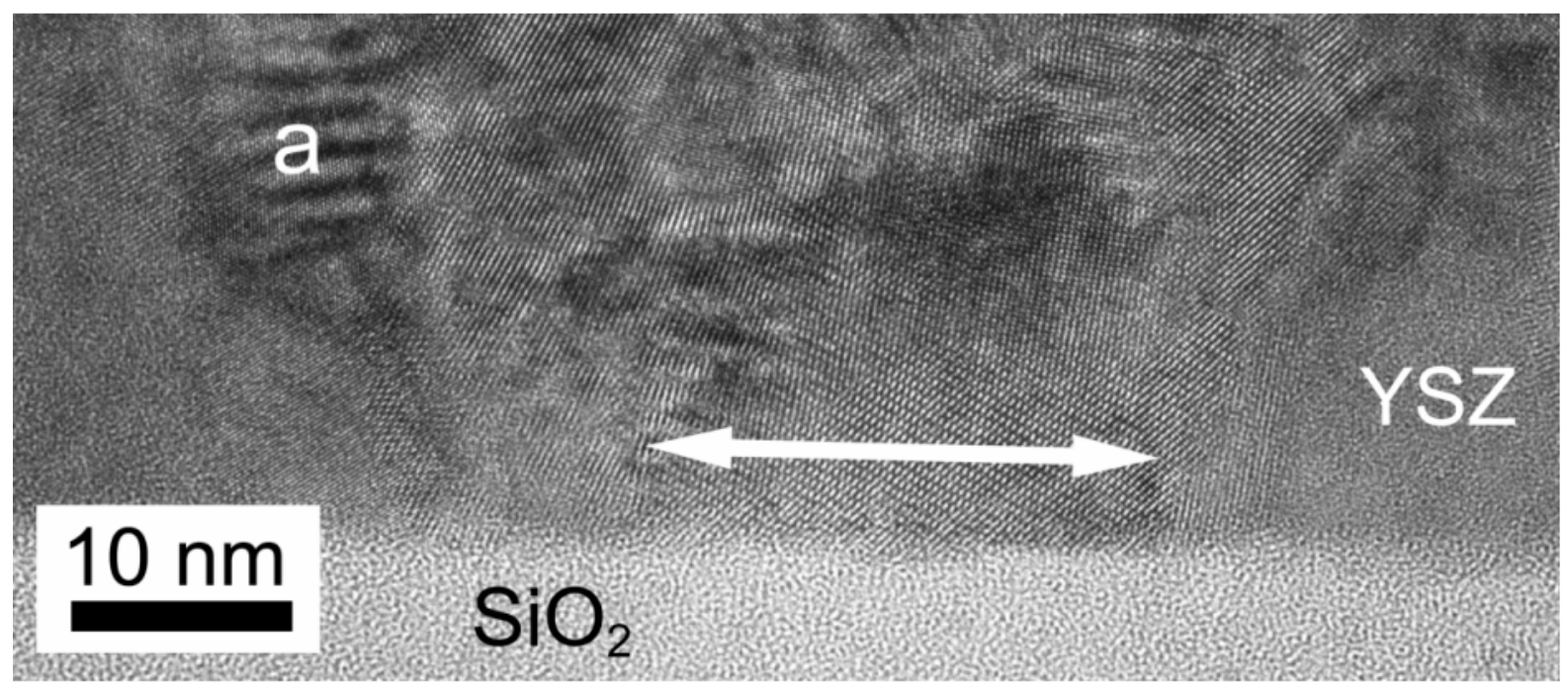

Abbildung 5.10: HREM-Querschnittsaufnahme von IBAD-YSZ auf Si, Blick senkrecht zur Wellenfront; Das Bild zeigt einen kristallinen Bereich, der direkt an der $\mathrm{SiO}_{2}$-Schicht anwächst. Das Moiré-Muster bei „a“ deutet auf übereinanderliegende kristalline Bereiche mit leichter Verdrehung hin.

\subsubsection{Einfluss der Stromdichte des unterstützenden Ionenstrahls auf die Anfangs- schicht}
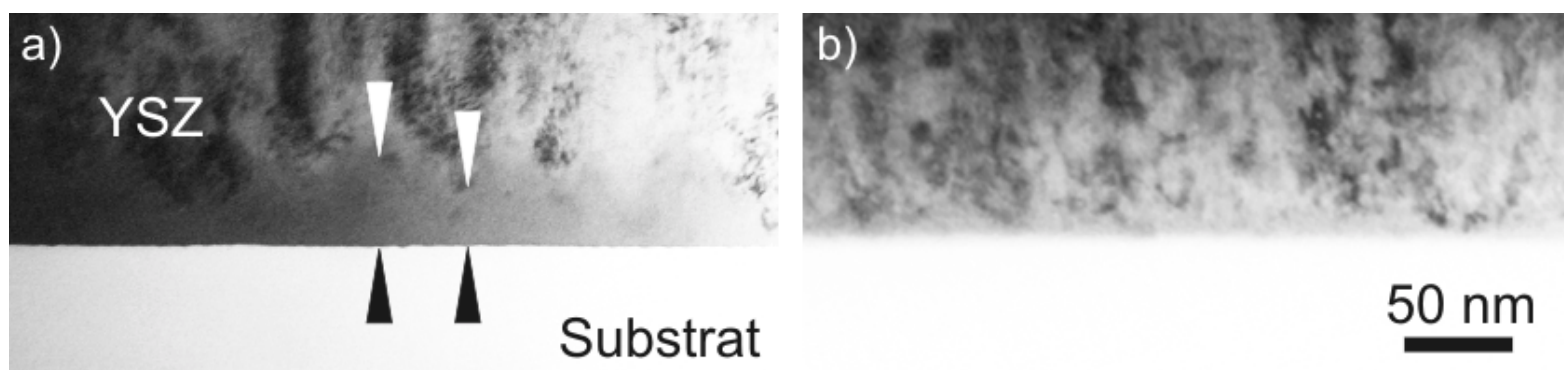

Abbildung 5.11: TEM-Querschnittsaufnahmen von YSZ-Filmen: a) YSZ-Film, abgeschieden ohne unterstützenden Ionenstrahl $(\eta=0)$, die Pfeile markieren den amorphen Bereich.

b) YSZ-Film, hergestellt mit unterstützendem Ionenstrahl $(\eta=3)$, es ist keine amorphe Anfangsschicht an der Substratoberfläche zu erkennen.

Die Dicke der amorphen Anfangsschicht hängt stark von der Stromdichte des unterstützenden Ionenstrahls ab. Dieses zeigt Abbildung 5.11 für ohne- $(\eta=0)$ bzw. mit Ionenstrahlunterstützung $(\eta=3)$ hergestellter YSZ-Filme. Ohne Ionenstrahlunterstützung variiert die Dicke der amorphen Schicht stark über den Beschichtungsbereich und kann bis zu $50 \mathrm{~nm}$ betragen 
(vgl. Abbildung 5.11 a). Selten existieren in diesen Filmen kristalline Bereiche direkt an der Substratoberfläche. Dagegen ist bei YSZ-Filmen, die mit unterstützenden Ionenstrahl $(\eta=3)$ abgeschieden wurden, keine amorphe Anfangsschicht zu erkennen (vgl. Abbildung $5.11 \mathrm{~b}$ ). Wie bereits in den vorherigen Abschnitten dargestellt ist der amorphe Bereich für $\eta=2$ ca. $10 \mathrm{~nm}$ dick.

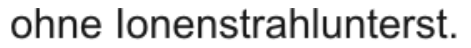

$\eta=0$
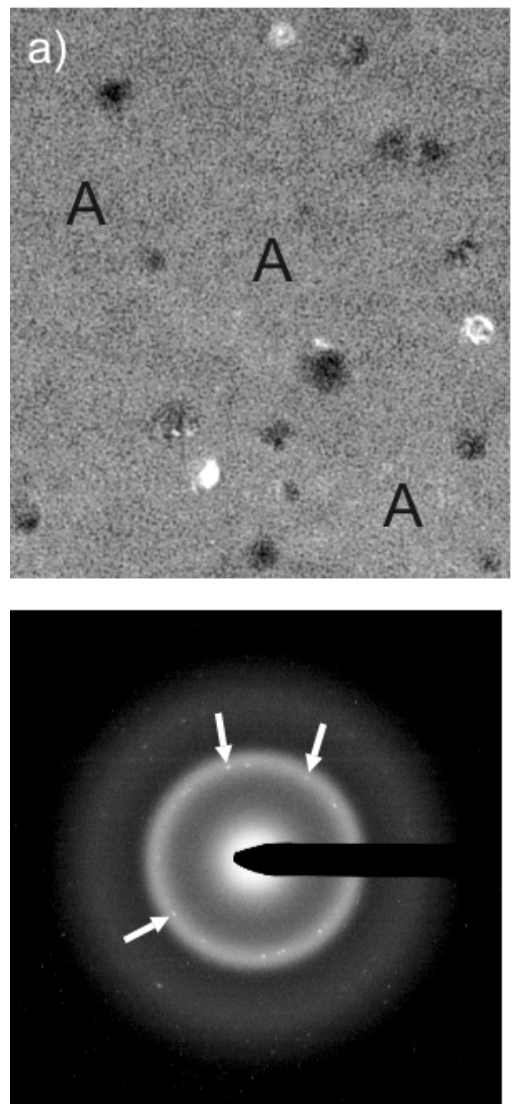

mit lonenstrahlunterst.

$$
\eta=2
$$
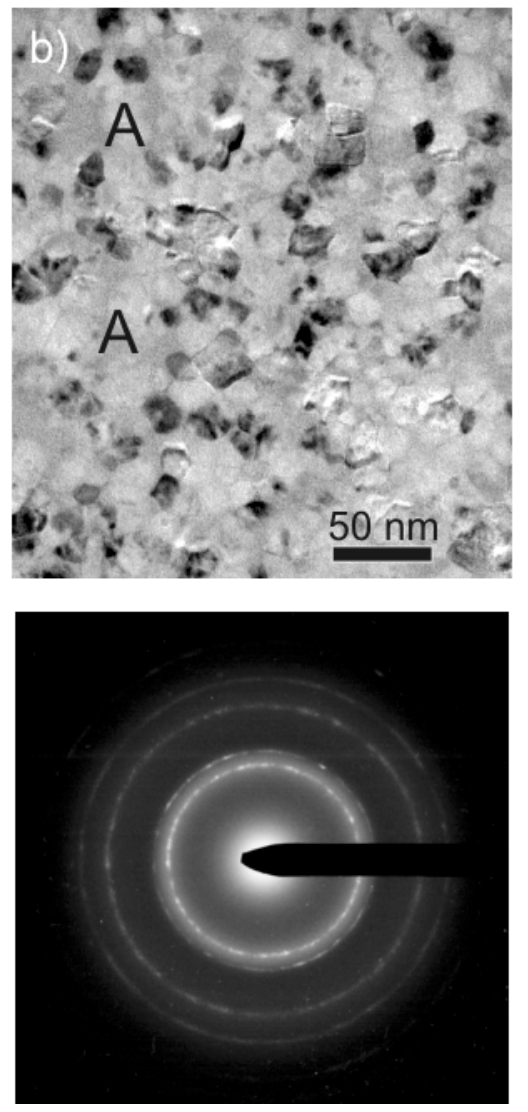

mit lonenstrahlunterst.

$$
\eta=4
$$
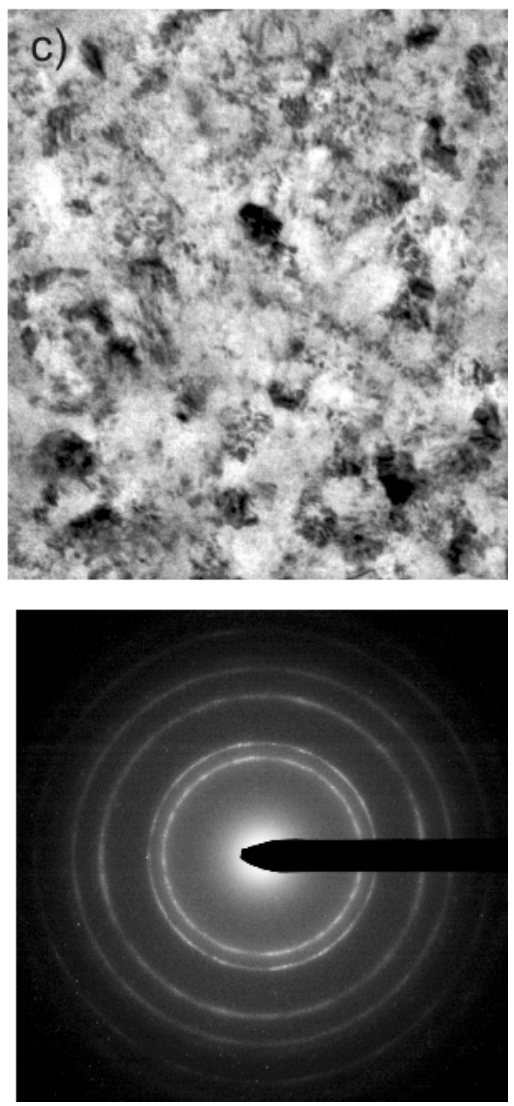

Abbildung 5.12: TEM-Übersichtsaufnahmen von ca. $30 \mathrm{~nm}$ dicken YSZ-Filmen auf mit Kohlenstoff bedampften Kupfernetzchen in Aufsicht, mit Feinbereichsbeugungsbildern des sichtbaren Bereiches. „A “ markiert im Realbild amorphe Bereiche. a) ohne unterstützenden Ionenstrahl abgeschieden; Aufgrund der geringen Anzahl der Kristallite zeigt das Feinbereichbeugungsbild neben den amorphen Ringen nur vereinzelt Reflexe, $b$ ) mit Ionenstrahlunterstützung $(\eta=2)$ abgeschieden, $c)$ mit Ionenstrahlunterstützung $(\eta=4)$ abgeschieden, es sind keine amorphen Bereiche im Realbild zu erkennen.

Die Zunahme des kristallinen Anteils mit zunehmendem $\eta$-Verhältnis zeigt Abbildung 5.12. Die mit „A“ markierten Bereiche zeigen amorphen Charakter in den TEM-Bildern. Die dazugehörenden Feinbereichsbeugungsbilder sind jeweils unter den Bildern zu sehen. Bei den YSZ-Filmen, hergestellt ohne unterstützenden Ionenstrahl, sind nur auf ca. 10\% der Fläche 
kristalline Bereiche zu erkennen. Dadurch erkennt man im Beugungsbild nur vereinzelte Reflexe neben den amorphen Ringen (Pfeile in Abbildung 5.12 a). Mit steigender Intensität des unterstützenden Ionenstrahls steigt die Anzahl der Beugungspunkte auf den Beugungsringen, also auch die Anzahl der Kristallite. Bei $\eta=2$ beträgt der amorphe Flächenanteil ca. 60 $70 \%$, bei $\eta=4$ verschwindet er. Die durchschnittlichen Korndurchmesser und Korndichte sind in Tabelle 5.3 eingetragen. Man erkennt, dass mit steigender Intensität des unterstützenden Ionenstrahls sowohl der durchschnittliche Korndurchmesser als auch die Korndichte ansteigt. In YSZ-Filmen, die bei $\eta=4$ abgeschieden wurden, ist die Bestimmung der Werte nicht möglich, da sich unter diesen Depositionsbedingungen in einem ca. $30 \mathrm{~nm}$ dicken YSZFilm eine vollständig kristalline Schicht ausbildet hat.

\begin{tabular}{|c|c|c|}
\cline { 2 - 3 } \multicolumn{1}{c|}{} & $\begin{array}{c}\text { durchschnittlicher } \\
\text { Korndurchmesser [nm] }\end{array}$ & $\begin{array}{c}\text { Durchschnittliche } \\
\text { Korndichte }\left[\mathrm{cm}^{-2}\right]\end{array}$ \\
\hline$\eta=0$ & 20 & $4 \times 10^{10}$ \\
\hline$\eta=2$ & 30 & $10 \times 10^{10}$ \\
\hline$\eta=4$ & $>30$ & - \\
\hline
\end{tabular}

Tabelle 5.3: Durchschnittliche Korndurchmesser, gemessen an YSZ-Filmen, die auf Kohlenstoff bedampften Kupfernetzchen bei verschiedenen $\eta$-Verhältnissen abgeschieden wurden.
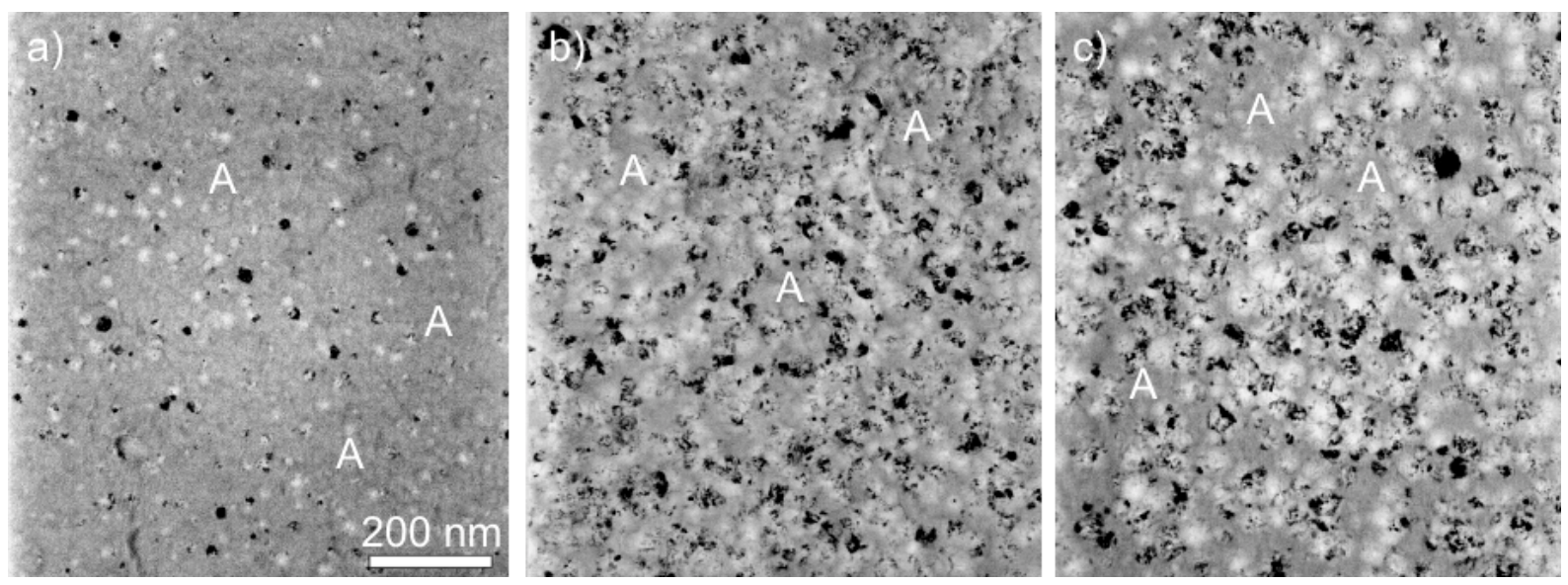

Abbildung 5.13: TEM-Übersichtsaufnahmen in Aufsicht an YSZ-Filme, ohne Ionenstrahlunterstützung abgeschieden auf mit Kohlenstoff beschichteten Kupfernetzchen, „A “ kennzeichnet amorphe Bereiche. a) ohne nachträglichen Ionenbeschuss, b) mit ca. 30 Sekunden -, c) mit ca. 60 Sekunden nachträglichen Ionenbeschuss mittels unterstützenden Ionenstrahl. 
Durch nachträglichen Ionenbeschuss kann die amorphe Anfangsschicht teilweise rekristallisiert werden (vgl. Abbildung 5.13). Teilbild Abbildung 5.13 a) zeigt den ursprünglichen YSZ-Film, b) einen YSZ-Film der nachträglich mit einer Ionendosis von ca. $5,6 \times 10^{16} \mathrm{~cm}^{-2}$ beschossen wurde. Der in Teilbild c) zu sehende Film wurde in doppelter Zeit (1 min) mit gleicher Strahlungsintensität beschossen, dies entspricht einer Ionendosis von $1,2 \times 10^{17} \mathrm{~cm}^{-2}$. Man erkennt, dass durch den nachträglichen Ionenbeschuss der Anteil der amorphen Bereiche (mit „A“ gekennzeichnet) reduziert wird. Die durchschnittlichen Korndurchmesser sind in Tabelle 5.4 aufgeführt.

Durch einen nachträglichen Ionenbeschuss wird also die Korngröße deutlich erhöht. Im Gegensatz zu einer ionenstrahlunterstützten Deposition werden aber keine neuen Kristallisationszentren erzeugt, da die mittlere Korndichte gleich bleibt.

\begin{tabular}{|c|c|c|c|}
\hline Dauer des nachträglichen Ionenbeschusses $t[\mathrm{~s}]$ & 0 & 30 & 60 \\
\hline$\Rightarrow$ Ionendosis $\left(n=\frac{J \cdot t}{e}\right)\left[\right.$ Ionen $\left./ \mathrm{cm}^{2}\right]$ & - & ca. $5,6 \times 10^{16}$ & ca. $1,2 \times 10^{17}$ \\
\hline \hline durchschnittlicher Korndurchmesser $[\mathrm{nm}]$ & 20 & 30 & 40 \\
\hline durchschnittlicher Korndichte $\left[\mathrm{cm}^{-2}\right]$ & \multicolumn{3}{|c|}{ ca. $4 \times 10^{10}$} \\
\hline
\end{tabular}

Tabelle 5.4: Durchschnittliche Korndurchmesser von ca. $30 \mathrm{~nm}$ dicken YSZ-Filmen, die nachträglich in verschiedenen Zeiten mittels des unterstützenden Ionenstrahls beschossen wurden.

\subsubsection{Kristallographische Orientierungen in der Anfangsschicht}

Um die Orientierungsverteilung der Körner in der Ebene in den Anfangsstadien des Filmwachstums zu charakterisieren, wurden an den Filmen, die auf mit Kohlenstoff beschichteten Kupfernetzchen abgeschieden wurden, in Aufsicht Feinbereichsbeugungsbilder aufgenommen. Ein typisches Feinbereichsbeugungsbild einer ca. $60 \mathrm{~nm}$ dicken IBAD-YSZ-Schicht zeigt Abbildung 5.14. Die feinkristalline Struktur ist an den diskreten Beugungspunkten auf den einzelnen Ringen zu erkennen. Für ein polykristallines Material ohne Vorzugsorientierung sollten alle Beugungsringe auftreten. Vorzugsorientierungen in der Ebene würden sich durch Häufungspunkte auf den einzelnen Beugungsringen bemerkbar machen. In Abbildung 5.15 sind die rechnerisch ermittelten Intensitäten der ersten vier Reflexe eines YSZPolykristalls aufgetragen [EMS Online]. In den Beugungsbildern ist aber zu beachten, dass die Intensitäten nicht allein durch die Strukturfaktoren wiedergespiegelt werden, so tragen z.B. zum (111)-Beugungsring die $\{111\}$-Ebenenscharen bei, die annähernd parallel zum einfallenden Elektronenstrahl orientiert sind. Dies ist z.B. bei (110)- und (112)-out-of-plane- 
Orientierungen der Fall. Sind Körner einer bestimmten out-of-plane-Orientierung häufig vertreten, macht sich dieses in einer höheren Intensität der angeregten Beugungsringe bemerkbar. Das in Abbildung 5.14 gezeigt Intensitätsprofil des Feinbereichsbeugungsbildes kann zwar nicht als repräsentativ angenommen werden, da die Intensität längs der ( $h k l)$-Ringe variiert und nicht unendlich viele Körner zum Beugungsbild beitragen, es wurde aber in allen aufgenommenen Beugungsbildern eine relativ hohe Intensität des (111)-Ringes beim gleichzeitigen Fehlen ausgeprägter Häufungspunkte beobachtet. Dieses kann als erster Hinweis auf eine erhöhte Anzahl von (110)-orientierten Kristalliten in der Anfangsschicht, die keine Vorzugsorientierung in der Ebene haben, gedeutet werden.
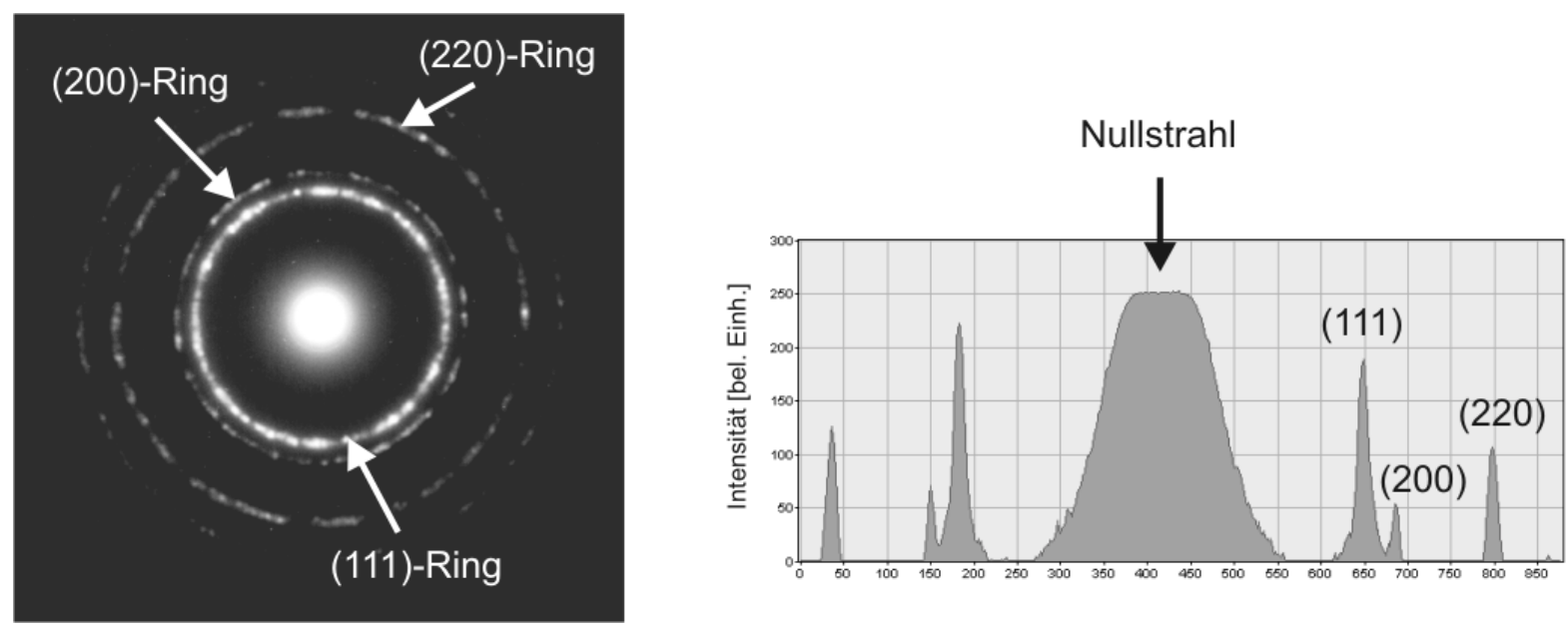

Abbildung 5.14: Feinbereichsbeugungsbild einer 60 nm dicken IBAD-YSZ-Schicht mit den gemessenen Intensitäten der Reflexringe. 


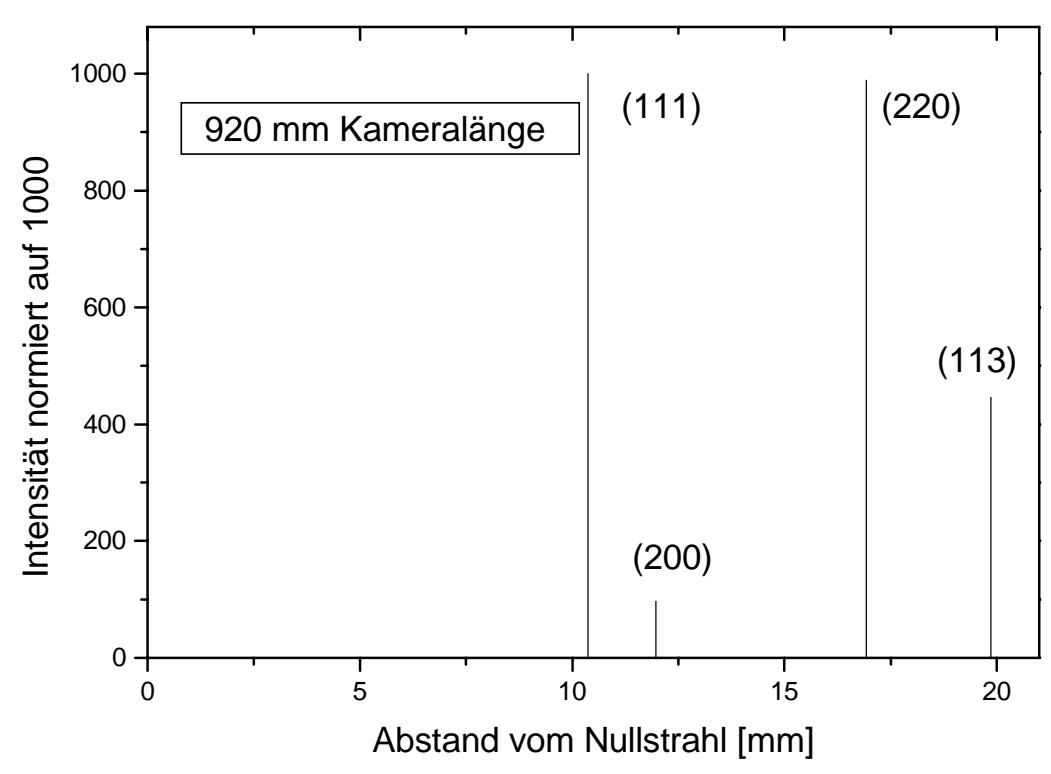

Abbildung 5.15: Berechnete Intensitäten der einzelnen TEM-Beugungsreflexe für eine YSZPulverprobe und deren Abstände zum Nullstrahl bei einer Kameralänge von $920 \mathrm{~mm}$ [EMS-Online].

Um dies weiter zu bestätigen, wurden zusätzlich zu den Beugungsexperimenten in den hochaufgelösten TEM-Aufsichtsbildern die out-of-plane-Orientierungen einzelner kristalliner Bereiche bestimmt. In Abbildung 5.16 ist eine HREM-Übersichtsaufnahme eines ca. $60 \mathrm{~nm}$ dicken YSZ-Films zu sehen. Da die einzelnen kristallinen Bereiche für eine genauere Orientierungsbestimmung mittels Feinstrahl-Beugung zu klein sind, wurde die Orientierung der einzelnen Kristallite nachträglich mittels der Fast-Fourier-Transformation (FFT) bestimmt. Es soll hier nochmals betont werden, dass man auf diese Weise nur Orientierungen von Bereichen identifizieren kann, in denen Netzebenen zu sehen sind. Zusätzlich muss das Beugungsmuster der FFT indiziert werden (vergleichbar mit dem eines Beugungsbildes). Bereiche ohne sichtbare Netzebenen können also sowohl amorph als auch kristallin sein. Im letzteren Fall reicht eine Verkippung des Kristallites um wenige Grad aus einer Zonenachse, um Netzebenen im HREM-Bild verschwinden zu lassen. 


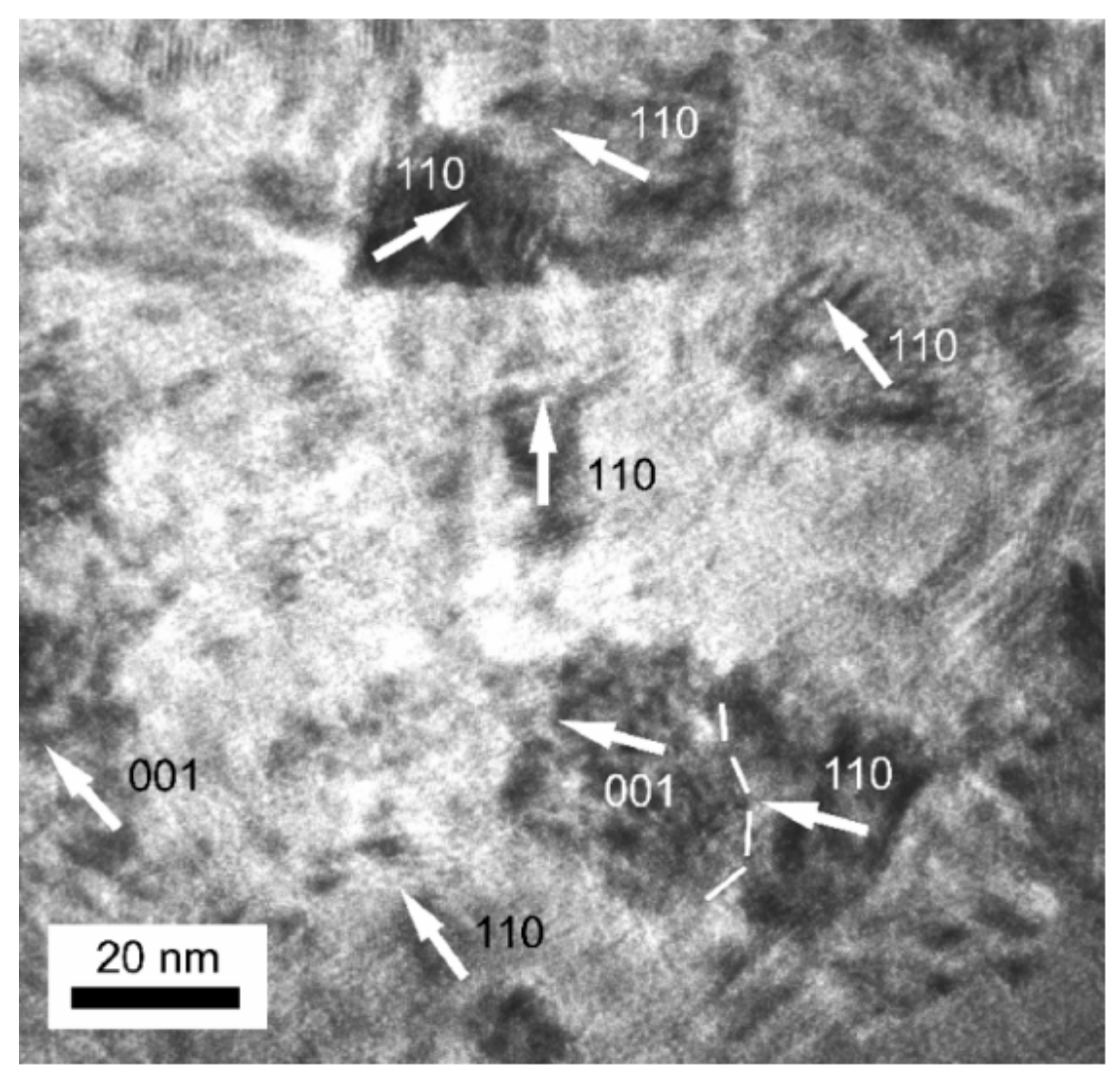

Abbildung 5.16: HREM-Aufsichtsaufnahme einer $60 \mathrm{~nm}$ dicken IBAD-YSZ-Schicht. Die outof-plane-Orientierungen der Kristallite sind angegeben, die Pfeile zeigen in <100>-Richtung und veranschaulichen die in-plane-Orientierung der jeweiligen Kristallite.

Um die Orientierung der Kristallite zu veranschaulichen, wurden in Abbildung 5.16 die indizierten Bereiche markiert. Die Indizes geben hierbei die out-of-plane-Orientierungen an, die Orientierungen in der Ebene sind durch Pfeile gekennzeichnet, die in die $<100>$-Richtung der einzelnen Körner zeigen. Auf dem Bild sind keine Anzeichen für eine Orientierungsbevorzugung in der Ebene zu erkennen. Mit einem Anteil von ca. 25 - 30\% sind (110)-orientierte Körner im Vergleich zu anders orientierten am häufigsten vertreten. Auch Körner mit einer (001)-Orientierung sind bei einer Schichtdicke von $60 \mathrm{~nm}$ vorhanden. Würde es sich hierbei um einen polykristallinen YSZ-Film handeln, sollten auch andere Orientierungen beobachtet werden. Neben den oben genannten Orientierungen wurden aber nur noch selten (111)- oder (113)-orientierte Körner gefunden. Andere Orientierungen traten nicht auf. Daher liegt die Vermutung nahe, dass es sich bei den Bereichen, deren Orientierungen aufgrund fehlender Netzebenen im hochaufgelösten TEM-Bild nicht bestimmbar sind, um (110)- bzw. (001)orientierte Körner handelt, die so verkippt sind, dass eine bzw. keine Netzebene parallel zum einfallenden Elektronenstrahl verläuft. Die Querschnittsuntersuchungen haben gezeigt, dass die bis zu $10 \mathrm{~nm}$ dicke amorphe Schicht an der Substratoberfläche in einer Filmdicke von 
$60 \mathrm{~nm}$ verschwunden sein muss, so dass es sich bei den Bereichen, die keine Netzebenen zeigen, nicht um amorphes Material handeln kann.

In YSZ-Filmen, die auf mit Kohlenstoff beschichteten Kupfernetzchen abgeschiedenen wurden, lassen sich anhand von Dunkelfeldaufnahmen die einzelnen Körner besonders gut unterscheiden und somit die Korngrößen bestimmen. Abbildung 5.17 zeigt die Verteilung der Korngröße für einen bei $\eta=2$ abgeschiedenen YSZ-Film. Die mittlere Größe der Körner in einer Filmdicke von 30 - $60 \mathrm{~nm}$ beträgt durchschnittlich ca. $30 \mathrm{~nm}$ (vgl. Abbildung 5.16).

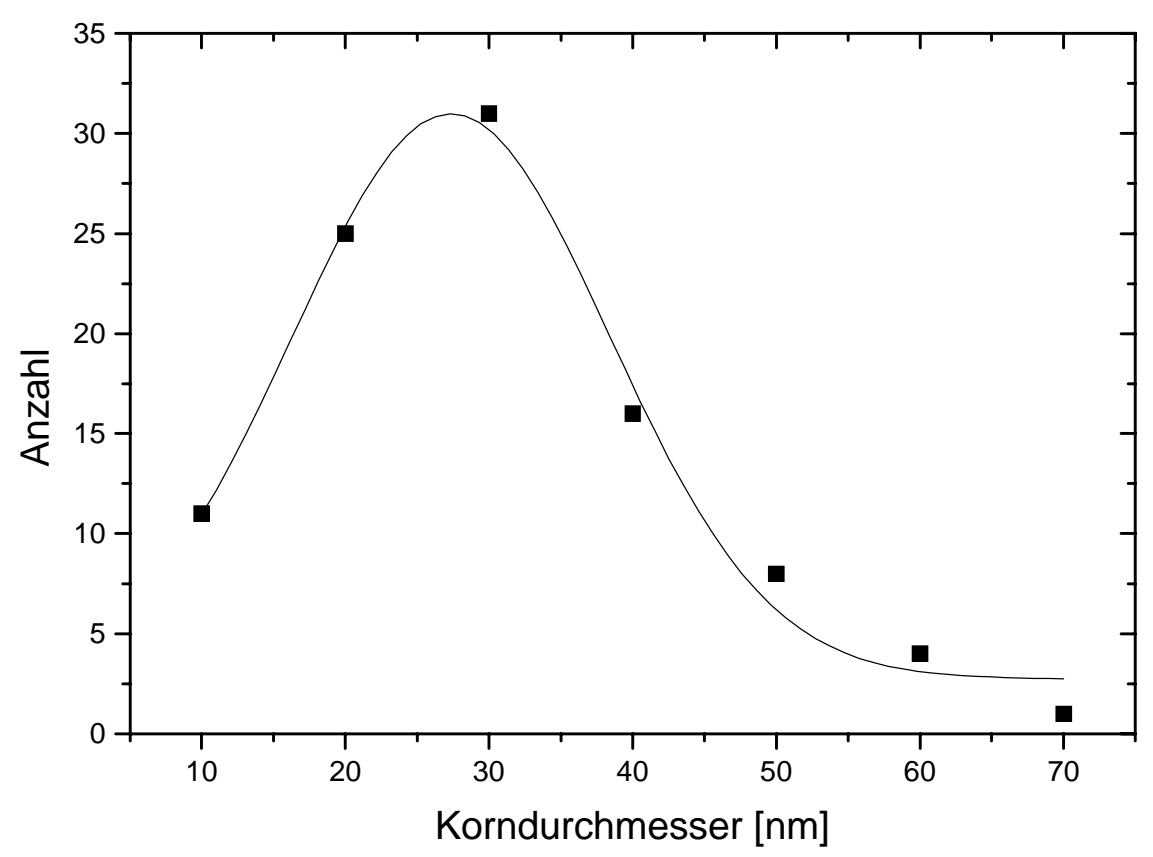

Abbildung 5.17: Verteilung der Korndurchmesser, gemessen in einem ca. 30 nm dicken YSZFilm. 

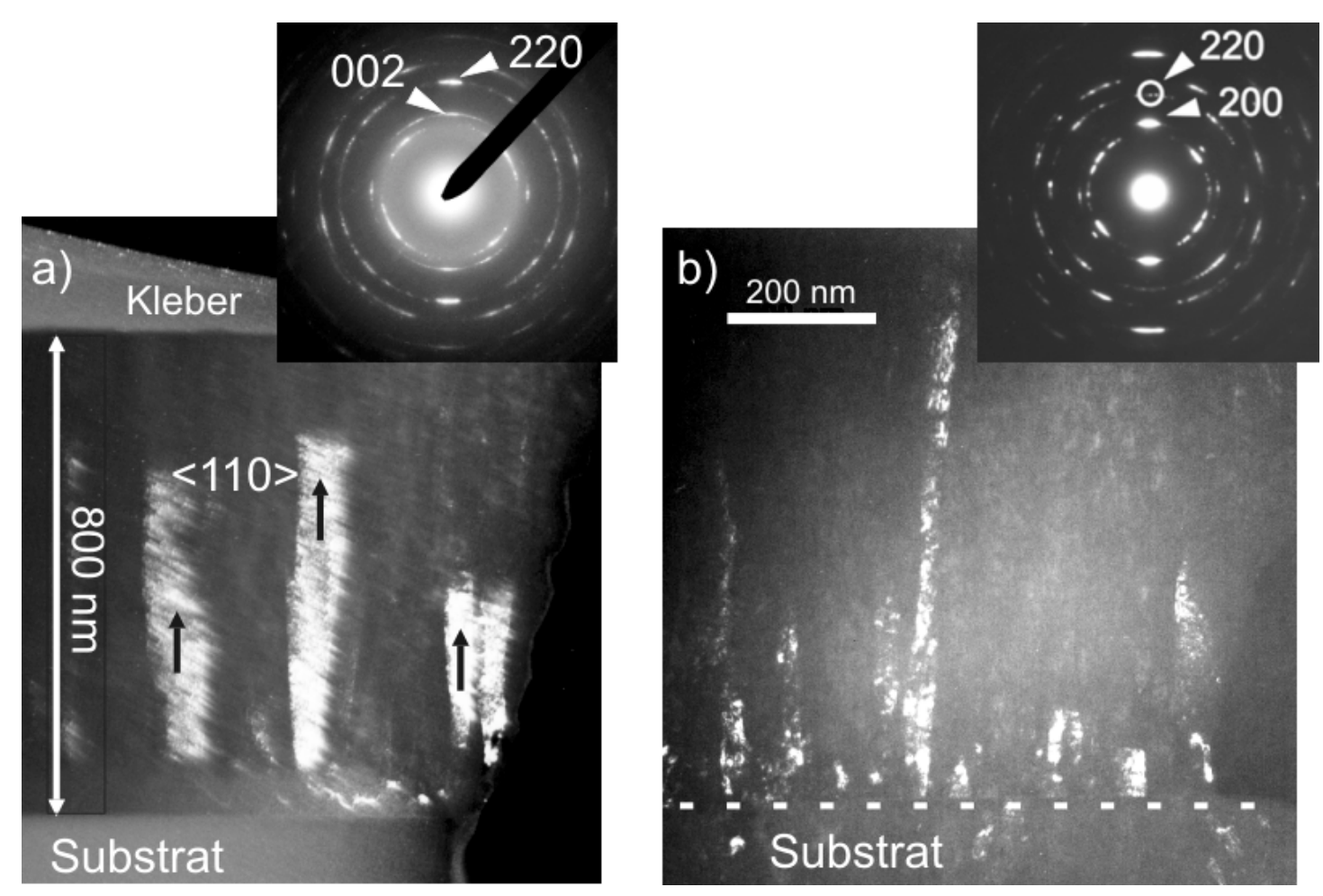

Abbildung 5.18: Dunkelfeldaufnahmen von YSZ-Filmen im Querschnitt, a) YSZ abgeschieden ohne unterstützenden Ionenstrahl, b) IBAD-YSZ, abgeschieden mit $\eta=2$, hell: (110)orientierte Körner. Beide Beugungsbilder zeigen Häufungspunkte auf den (220)-Ringen in Wachstumsrichtung. Mit steigender Intensität des unterstützenden Ionenstrahls nimmt der (110)-Anteil im IBAD-YSZ-Film ab.

Der hohe Anteil an (110)-orientierten Bereichen wird in Querschnittsaufnahmen bestätigt. Feinbereichsbeugungsbilder (Abbildung 5.18), die nahe des Substratrandes erstellt wurden, zeigen Häufungspunkte auf den (200)- und (220)-Ringen in Richtung der Substratnormalen für YSZ-Filme, hergestellt ohne- und mit Ionenstrahlunterstützung. Für die in Abbildung 5.18 dargestellten Dunkelfeldaufnahmen wurde die Position der Kontrastblende so gewählt, dass Bereiche, deren (110)-Ebenennormalen parallel zur Substratnormalen verlaufen, hell zu erkennen sind. Neben einer Vielzahl von kleinen (110)-Kristalliten oberhalb der Substratoberfläche bzw. amorphen Anfangsschicht, fallen in den Dunkelfeldabbildungen ausgeprägte (110)-Wachstumssäulen auf. Über die in-plane-Textur dieser Bereiche kann in beiden Bildern keine Aussage gemacht werden. Die an der Substratoberfläche, der bei $\eta=2$ abgeschiedenen YSZ-Filme, 10 - $30 \mathrm{~nm}$ breiten (110)-orientierten Körner wachsen säulenartig bis zu einer Schichtdicke von $50-150 \mathrm{~nm}$ (vgl. Abbildung 5.18). Vereinzelt weiterwachsende (110)orientierte Körner bilden eine Ausnahme. Der Anteil der (110)-orientierten Säulen ist bei YSZ-Filmen, die ohne unterstützenden Ionenstrahl hergestellt wurden ca. 40\% und somit höher als bei YSZ-Filmen, die unter Ionenstrahlunterstützung abgeschieden wurden. Die Breite der (110)-Säulen beträgt hier im unteren Bereich des Films ca. $50 \mathrm{~nm}$ und steigt auf ca. $90 \mathrm{~nm}$ 
an. Ab einer Filmdicke von $400-600 \mathrm{~nm}$ sind keine (110)-orientierten Bereiche mehr zu finden. Bei den mit hoher Intensität des unterstützenden Ionenstrahls hergestellten Filmen $(\eta=3)$ konnten keine (110)-orientierte Säulen sichtbar gemacht werden, da der Anteil zu gering ist.

Die in diesem Abschnitt durchgeführten Experimente zeigten, dass die YSZ-Filme durch eine amorphe Anfangsschicht charakterisiert sind, wobei die Dicke dieser Schicht mit zunehmendem $\eta$-Verhältnis geringer wird. Oberhalb der Anfangsschicht findet man im wesentlichen (110)- und (001)-orientierte Kristallite, die keine in-plane-Textur aufweisen. Die Größe und der Anteil der (110)-orientierten Säulen nimmt mit größer werdenden $\eta$-Verhältnissen ab, wodurch die (220)-Reflexe in den Röntgendiffraktogrammen im Vergleich zu den (200)Reflexen verschwindend gering werden.

\section{$5.4 \quad$ Ausrichtung der (001)-Textur in YSZ-Filmen}

Die Ergebnisse des letzten Abschnittes haben gezeigt, dass selten Körner in den ersten Nanometern in Bezug auf den unterstützenden Ionenstrahl und der Substratnormalen schon biaxial ausgerichtet sind. Im Verlauf des weiteren Filmwachstums muss, wie die röntgenografischen Ergebnisse zeigen, sich sowohl die in-plane-als auch die out-of-plane-Komponente der Textur durch den Ionenbeschuss oder letztere auch durch die Wachstumsrichtung des Filmes einstellen. Es ist weiterhin bekannt, dass sich die out-of-plane-Textur bereits bei geringen Filmdicken einstellt, während sich die in-plane-Textur erst langsam mit zunehmender Filmdicke verbessert. Wie es zunächst zu der gewünschten (001)-Ausrichtung kommen kann, soll im folgenden gezeigt werden.

\subsubsection{Entwicklung der Texturen in unter IBAD-Bedingungen abgeschiedenen YSZ- Filmen}

Um die Entwicklung der Textur, der bei $\eta=2$ abgeschiedenen YSZ-Filme zu untersuchen, wurden Beugungsexperimente an im Querschnitt präparierten Proben durchgeführt. Abbildung 5.19 zeigt ein Feinbereichsbeugungsbild der ersten ca. $300 \mathrm{~nm}$ des YSZ-Films. Es zeigt schon einen Filmanteil mit (001)-Textur, deutlich zu erkennen an den Häufungspunkten auf den (002)- bzw. (004)-Ringen in Wachstumsrichtung (Abbildung 5.19, links). Die Probe wurde für die Aufnahme so präpariert, dass man senkrecht zur Projektion des Ionenstrahls blickt und so bei der gewünschten (001)-Textur in $<110>$-Richtung des YSZ sieht. Für diese Blickrichtung ist zum Vergleich ein berechnetes Beugungsbild für eine einkristalline Probe gezeigt (Abbildung 5.19, rechts). Die Breite des (002)-Häufungspunktes entspricht einer Verkippung der (001)-orientierten Körner aus der Substratebene um den einfallenden Elektronenstrahl. Der Winkel dieser Verkippung beträgt etwa $\pm 10^{\circ}$. Die in-plane-Textur kann aus diesem Beugungsbild zwar nicht bestimmt werden, aber die hohe Intensität der (111)-Reflexe und 
insbesondere die geringe Intensität der (020)- bzw. (020) -Reflexe sind ein deutlicher Hinweis auf eine solche Textur.
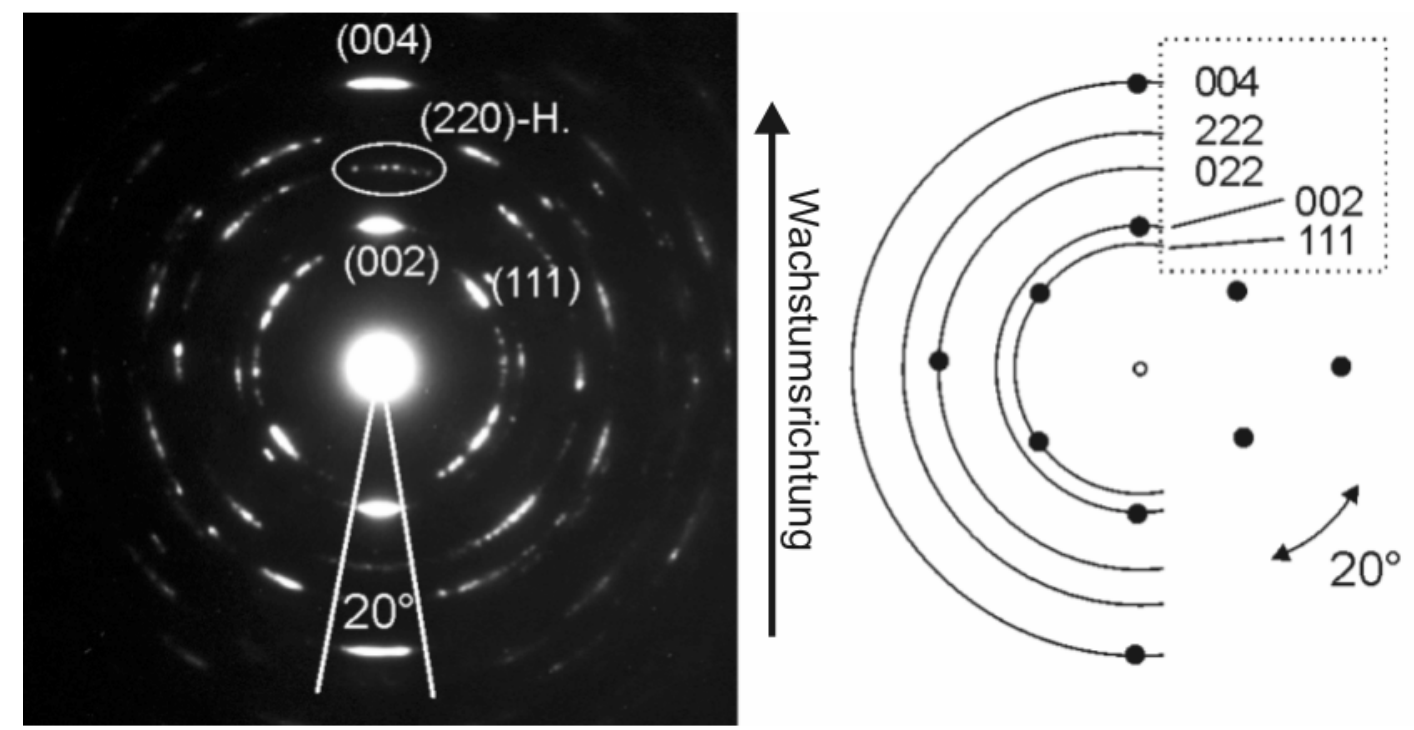

Abbildung 5.19: rechts: Feinbereichsbeugungsbild der ersten ca. $300 \mathrm{~nm}$ YSZ-Filmdicke im Querschnitt. Häufungspunkte auf den Beugungsringen in Wachstumsrichtung deuten auf eine Textur hin. links: Indizierung der einzelnen Ringe, mit Beugungspunkten des [110]-Pols. 

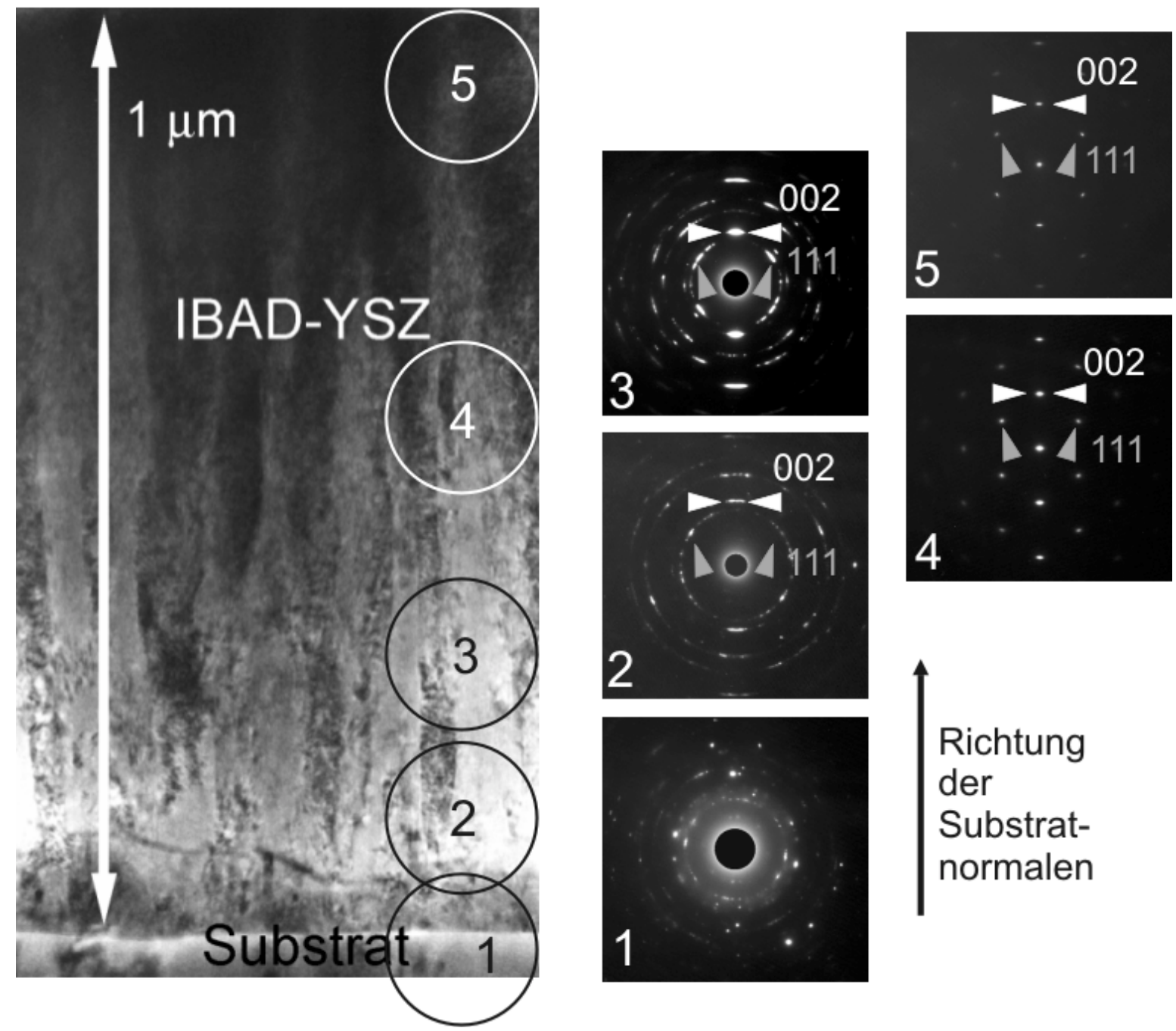

Abbildung 5.20: TEM-Querschnittsaufnahme eines IBAD-YSZ-Films mit einer Serie von Feinbereichsbeugungsbildern, die an den markierten Stellen aufgenommen wurden. Man blickt senkrecht zur Projektion des unterstützenden Ionenstrahls.

Abbildung 5.20 zeigt nun eine typische Folge von Feinbereichsbeugungsbildern, die in unterschiedlichen Abständen zum Substrat erstellt wurden. Der Durchmesser des analysierten Bereichs betrug dabei ca. $200 \mathrm{~nm}$, dem Durchmesser der SAD-Blende (ㅌelected Area Defraction). In den Beugungsbildern sind die relevanten Beugungspunkte bzw. -ringe mit Pfeilen markiert. Der Nullstrahl wurde teilweise ausgeschnitten, er spielt für die weitere Betrachtung keine Rolle. Beugungsbild 1 wurde direkt an der Substratoberfläche aufgenommen. Es zeigt sowohl Reflexe des polykristallinen Substrats als auch einzelne Beugungsringe des IBADYSZ. In diesem Beugungsbild sind keine Merkmale einer Textur zu erkennen. Dagegen erkennt man in Beugungsbild 2, aufgenommen in einer Schichtdicke von ca. $200 \mathrm{~nm}$, Häufungspunkte auf dem (002)-Ring in Richtung der Substratnormalen (weiße Pfeile), die auf eine (001)-Textur hinweisen. Diese Textur ist im Beugungsbild 3 schon deutlich ausgeprägter. 
Die Positionen und Intensitäten der Beugungspunkte auf den folgenden Beugungsbildern 4 und 5 entsprechen schon einem [011]-Beugungsbild (vgl. Abbildung 5.19, links). Bei einer Filmdicke von max. $500 \mathrm{~nm}$ ist bei den gewählten Abscheidebedingungen $(\eta=2)$ die Ausbildung der (001)-out-of-plane-Textur im wesentlichen abgeschlossen. Sie hat einen Wert von ca. $\pm 2-3^{\circ}$ erreicht.

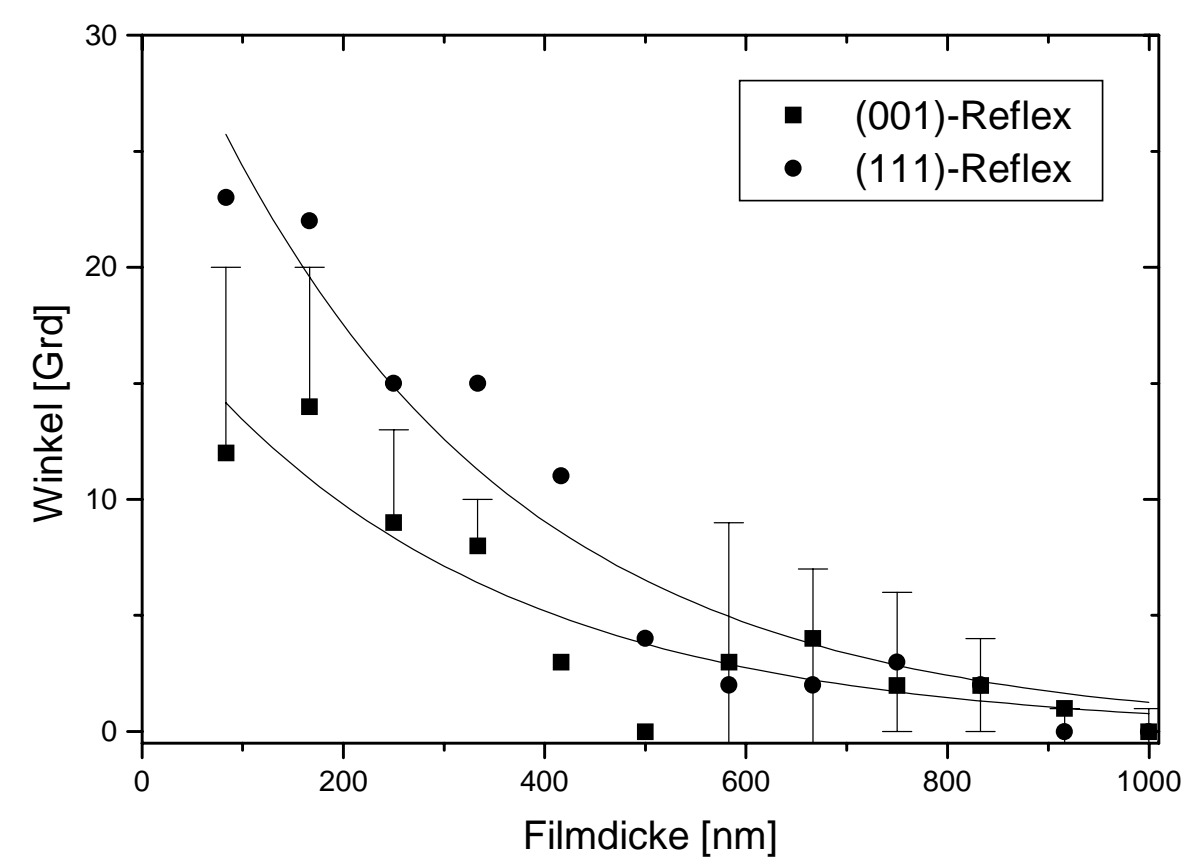

Abbildung 5.21: Änderung der (001)-Orientierung mit zunehmender Filmdicke. Die Fehlerbalken sind exemplarisch eingezeichnet, halbe Fehlerbalken deuten hier die obere Grenze der ermittelten Werte an.

In einem zu Abbildung 5.20 analogen Experiment wurde die Probe im Mikroskop so bewegt, dass der fokussierte Elektronenstrahl parallel zur Substratnormalen entlanglief. Die Änderung des Beugungsbildes wurde mit einer Digitalkamera aufgenommen. Anschließend wurden in den einzelnen Beugungsbilder der Winkel der (001)-Häufungspunkte zum Nullstrahl ausgemessen. Zum Vergleich wurde zusätzlich die Winkelbreite eines (111)-Häufungspunkt gemessen, da diese durch den Strukturfaktor heller und somit einfacher zu bestimmen waren (vgl. Abbildung 5.15, Seite 36). Die Auswertung der einzelnen Bilder zeigt Abbildung 5.21. Die Winkelverteilung der Körner an der Filmoberseite relativ zur Substratnormalen beträgt ca. $1^{\circ}$, wobei allerdings bei einem Strahldurchmesser von ca. $100 \mathrm{~nm}$ und einem durchschnittlichen Korndurchmesser von ca. $100 \mathrm{~nm}$ an der Filmoberseite in diesem Fall nur zwei Körner $\mathrm{zu}$ einer Verbreiterung des Beugungspunktes beitragen können. Dieses Ergebnis verdeutlicht 
nochmals, dass ab einer Filmdicke von ca. $500 \mathrm{~nm}$ keine große Verbesserung der out-ofplane-Textur mehr erfolgt.

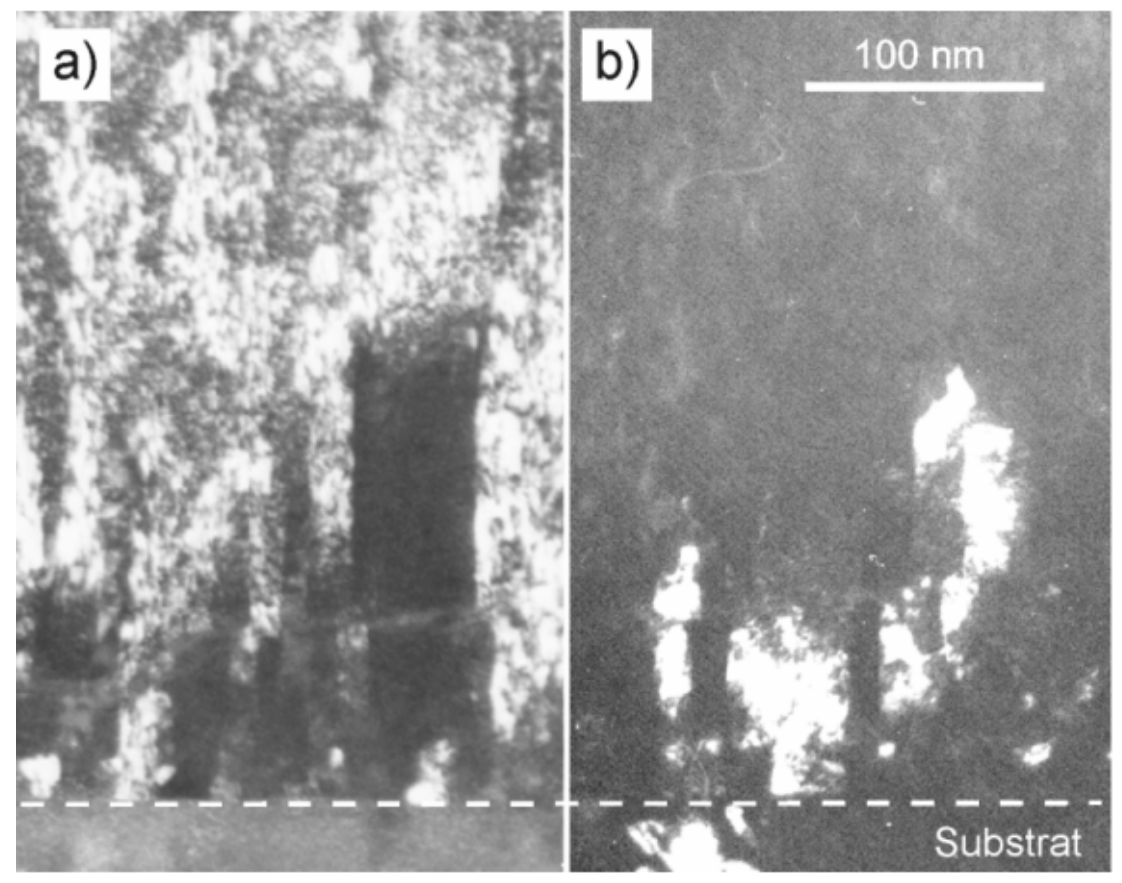

Abbildung 5.22: Querschnitts-Dunkelfeldaufnahmen von IBAD-YSZ, in beiden Aufnahmen ist die selbe Probenstelle zu sehen. Hell in a) (001)-texturierte Bereiche, in b) (110)-texturierte Bereiche

Dies zeigt sich auch, wenn man die Schichtdickenabhängigkeit des Anteils (110)orientierter Bereiche im Film untersucht. Abbildung 5.22 zeigt zwei Dunkelfeldaufnahmen eines IBAD-YSZ-Films im Querschnitt. Es ist jeweils dieselbe Probenstelle zu sehen. Für diese Aufnahmen wurde die Position der Kontrastblende so gewählt, dass in a) (001)orientierte- bzw. in b) (110)-orientierte Körner als helle Bereiche zu erkennen sind. Die Größe der Blende bestimmt hierbei den Bereich der Verkippung der jeweiligen Orientierungen aus der Substratebene innerhalb dessen ein Filmbereich noch als (001)- bzw. (110)-orientiert identifiziert wird. In diesem Fall beträgt sie ca. $\pm 1^{\circ}$. Die Zunahme des (001)-texturierten IBADYSZ-Anteils in den ersten $300 \mathrm{~nm}$ ist deutlich zu sehen. Oberhalb dieser Filmdicke ist die (001)-Textur sehr ausgeprägt, (110)-orientierte Bereiche treten nicht mehr auf.

Die Unterschiede in den Feinbereichsbeugungsbildern 2 und 3 in Abbildung 5.20 (Seite 42) zeigen, dass ab einer Filmdicke von ca. $300 \mathrm{~nm}$ die Ausbildung einer ausgeprägten in-planeTextur einsetzt. Nachdem sich also in diesem Wachstumsstadium eine (001)-out-of-planeTextur gebildet hat, folgt nun mit zunehmender Filmdicke eine Änderung der Orientierung in der Ebene. 


\subsubsection{Einfluss der Stromdichte des unterstützenden Ionenstrahls}

Analog zu dem Experiment in Abbildung 5.20 wurde die out-of-plane-Texturentwicklung in YSZ-Filmen untersucht, die mit $\eta=0$ bzw. $\eta=3$ abgeschieden wurden (vgl. Abbildung 5.23 und Abbildung 5.24). Die TEM-Ergebnisse sollen an dieser Stelle mit den röntgenographischen Ergebnissen verglichen werden.

YSZ-Film, abgeschieden ohne lonenstrahlunterstützung

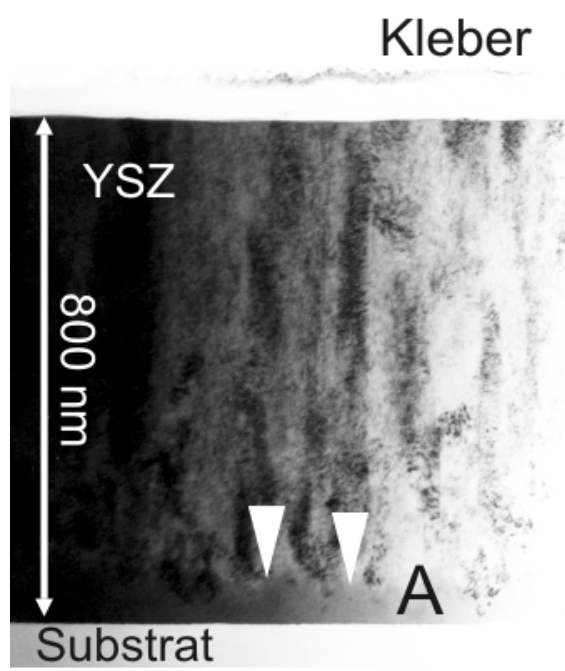

a)

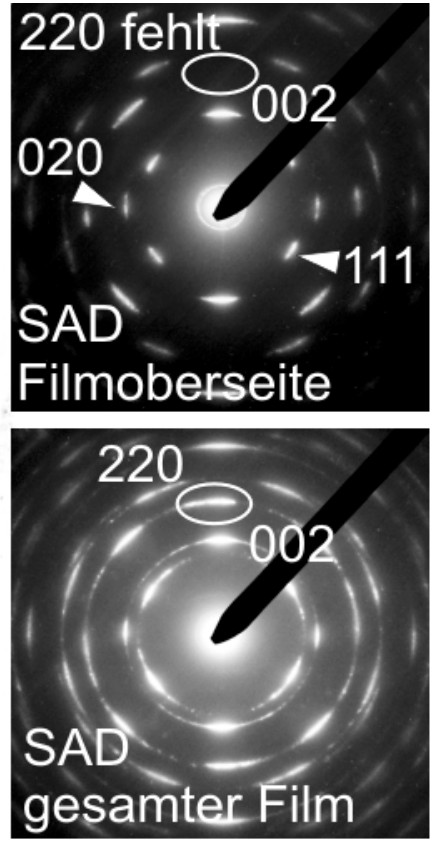

b)

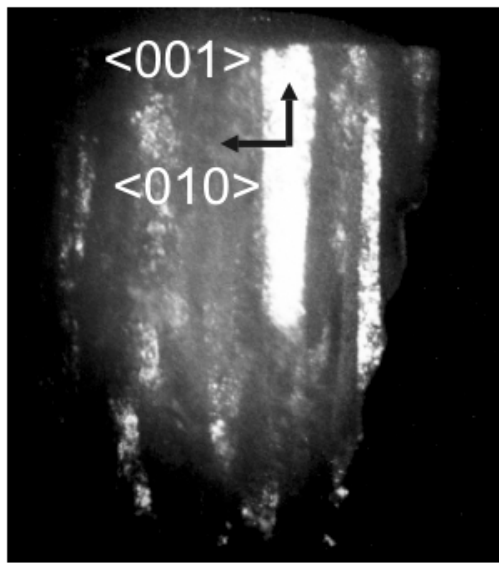

c)

Abbildung 5.23: Entwicklung der(001)-Textur in YSZ-Filmen, hergestellt ohne IBAD $(\eta=0)$.

a) TEM-Querschnittsaufnahme: Die Pfeile markieren einen bis zu $50 \mathrm{~nm}$ dicken amorphen Bereich, auf dem kristalline Säulen wachsen. b) Feinbereichsbeugungsbilder des gesamten Films zeigen einen hohen Anteil an (110)-orientierten Säulen, die an der Oberseite nicht mehr zu finden sind. Die (020)-Reflexe senkrecht zu Wachstumsrichtung deuten auf keine in-planeTextur hin. c) Dunkelfeldaufnahme, in der (001)-orientierte Säulen, die um $45^{\circ}$ zu den Nachbarsäulen in-plane verdreht sind, zu sehen sind. 


$$
\eta=3
$$
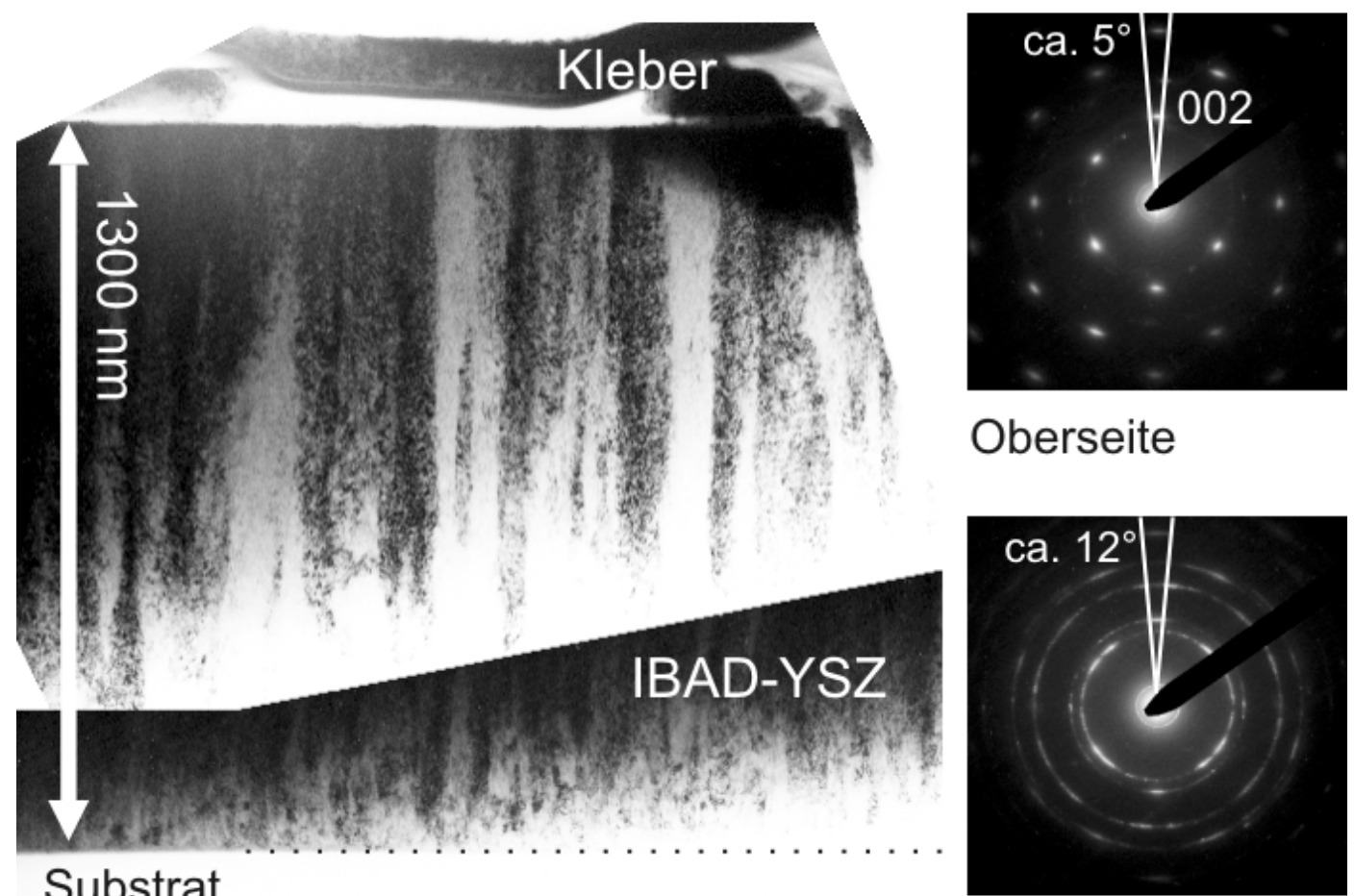

\section{Oberseite}

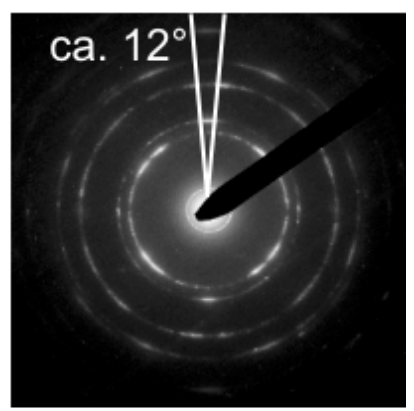

\section{Unterseite}

Abbildung 5.24: TEM-Querschnittsaufnahme eines mit erhöhter Stromdichte des unterstützenden Ionenstrahls hergestellten YSZ-Films, Montage. Das Bild wurde aus zwei Aufnahmen zusammengesetzt, um Ober- und Unterseite in einem Bild zu zeigen.

Der YSZ-Film, der ohne Ionenstrahlunterstützung abgeschieden wurde, hat eine dickenunabhängige out-of-plane-Textur von ca. $8^{\circ}$ (FWHM) (vgl. Tabelle 5.1, Seite 25). Diese dickenunabhängige out-of-plane-Textur wurde auch im TEM beobachtet. Der große Unterschied zwischen der Texturangabe der Röntgenmessungen und der aus den Feinbereichsbeugungsbildern ermittelten Breite der Häufungspunkte von ca. $20^{\circ}$ ist dadurch zu erklären, dass bei Röntgenmessungen die Halbwertsbreite des Reflexes angegeben wird, dagegen wird bei den TEMUntersuchungen die gesamte Breite eines $(h k l)$-Reflexes bestimmt. Da diese Breite auch von der Belichtungszeit des Beugungsbildes abhängig ist, wurde die Belichtungszeit immer so gewählt, dass man davon ausgehen kann, dass die Breite annähernd maximal abgebildet wurde. In Abbildung 5.25 ist der Unterschied der jeweiligen Messmethode schematisch dargestellt. 


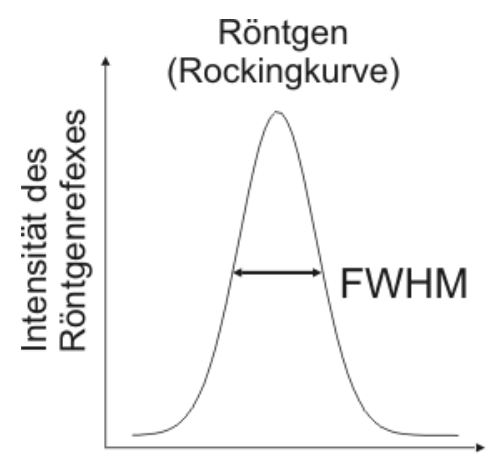

$\Theta[\mathrm{Grd}]$

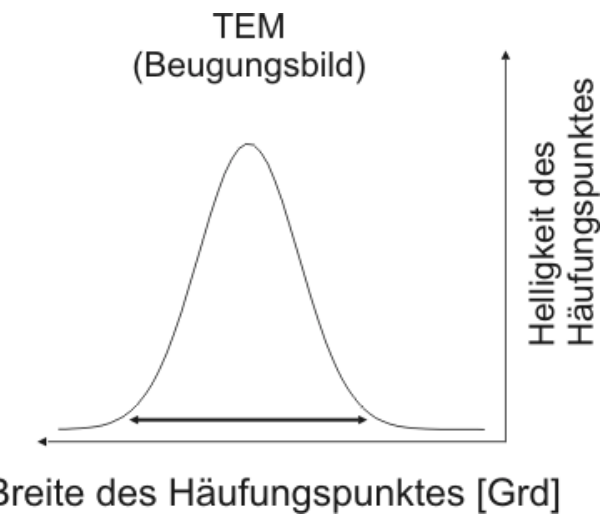

Breite des Häufungspunktes [Grd]

Abbildung 5.25: Schematische Darstellung der Röntgen- und der TEM-Texturangabe. Die Breite des Häufungspunktes ist von der Belichtungszeit abhängig!

Für YSZ-Filme, die mittels Ionenstrahlunterstützung hergestellt wurden, ist die out-ofplane-Textur dickenabhängig, das zeigen Röntgenmessungen an verschieden dicken YSZFilmen (vgl. Tabelle 5.1, Seite 25). Die Entwicklung der Textur wurde anhand von Feinbereichsbeugungsbilder an Querschnittsproben in verschiedenen Filmdicken bestimmt. Die Werte sind in Tabelle 5.5 angeführt. Für YSZ-Filme, die bei $\eta=2$ abgeschieden wurden, ist ab einer Filmdicke von ca. $500 \mathrm{~nm}$ keine signifikante Verbesserung zu verzeichnen. Ist $\eta=3$ beträgt diese Filmdicke ca. $300 \mathrm{~nm}$.

\begin{tabular}{|c|c|c|}
\hline $\begin{array}{c}\text { Breite des (001)- } \\
\text { Häufungspunktes... }\end{array}$ & $\eta=2$ & $\eta=3$ \\
\hline ..in Substratnähe & ca. $20^{\circ}$ & ca. $12^{\circ}$ \\
\hline $\begin{array}{c}\text {...in Oberflächennähe eines } 1 \mu \mathrm{m} \\
\text { dicken YSZ-Films }\end{array}$ & ca. $5-6^{\circ}$ & ca. $5^{\circ}$ \\
\hline & $\begin{array}{c}\text { kaum Änderung ab } \\
\text { ca. } 500 \mathrm{~nm}\end{array}$ & $\begin{array}{c}\text { kaum Änderung ab } \\
\text { ca. } 300 \mathrm{~nm}\end{array}$ \\
\hline
\end{tabular}

Tabelle 5.5: Breite des (001)-Häufungspunktes der Feinbereichsbeugungsbilder, gemessen in verschiedenen Filmdicken an unter verschiedenen $\eta$-Verhältnissen abgeschiedener YSZ-

Filme. 


\section{Hochauflösende Untersuchungen zur Texturausbildung}

Im folgenden werden verschiedene Beobachtungen zur Texturentstehung zur Vertiefung des vorhergehenden Abschnitts vorgestellt. Dabei werden nur Ergebnisse der Untersuchungen von IBAD-YSZ-Filmen gezeigt, die mit Ionenstrahlunterstützung mit $\eta=2$ hergestellt wurden.

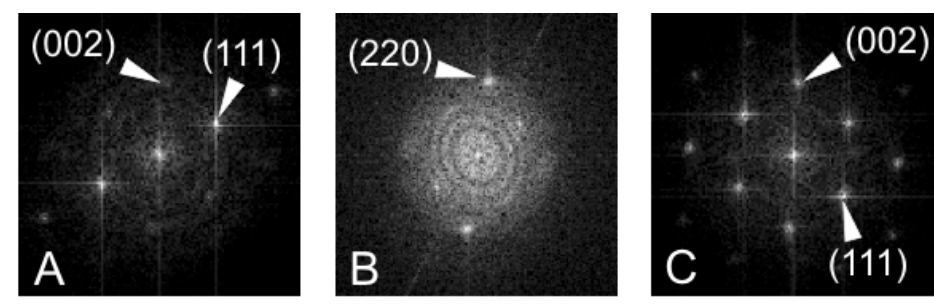

Fast Fourier-Transformationen der Bereiche A - C (Markierung)

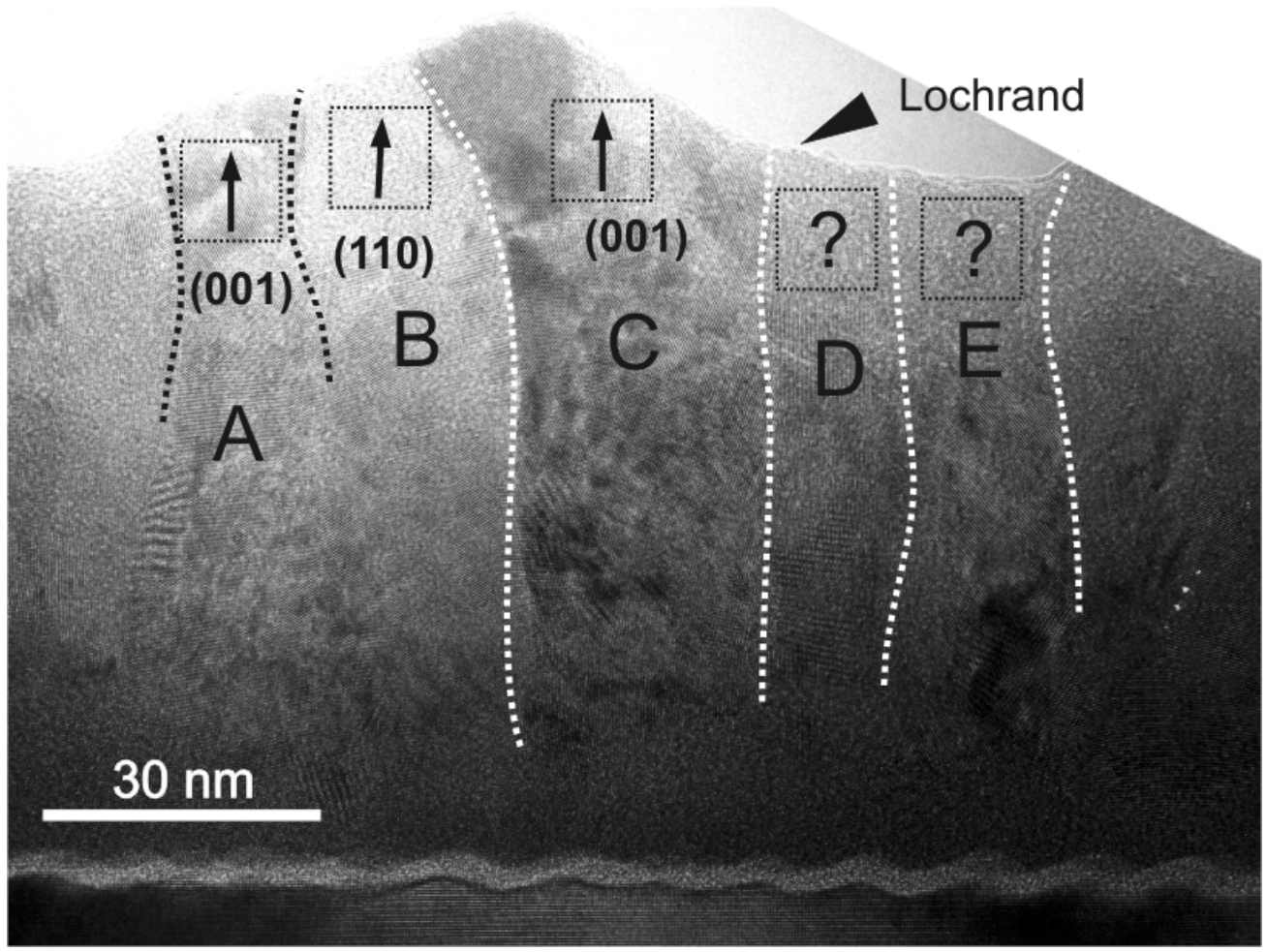

Abbildung 6.1: HREM-Aufnahme von IBAD-YSZ auf Silizium. Man sieht die ersten $100 \mathrm{~nm}$ des IBAD-YSZ-Films im Querschnitt. Die FFTs der markierten Bereiche sind oben abgebildet. Die Indizierung gibt Aufschluss über die out-of-plane-Orientierung. 
Zum Vergleich zu dem Aufsichtbild in Abbildung 5.16 (Seite 37) zeigt Abbildung 6.1 eine HREM-Aufnahme des IBAD-YSZ-Films im Querschnitt. Die in der Abbildung markierten Säulen wurden anhand der unterschiedlichen Kontraste gekennzeichnet. Sie haben eine laterale Ausdehnung von bis zu $30 \mathrm{~nm}$. Um die Orientierung der kristallinen Bereiche zu bestimmen, wurden Fast-Fourier-Transformationen in den markierten Zonen durchgeführt. So ist z.B. der mit B gekennzeichnete Bereich (110)-orientiert. Die Orientierung der Nachbarbereiche (A und C) ist jeweils (001), wobei die Ebenennormalen teilweise um einige Grad aus der Substratnormalen verkippt sind. Auch liegen die Normalen nicht immer in der Bildebene. Die Orientierungen der Bereiche $\mathrm{C}$ und $\mathrm{D}$ konnten nicht bestimmt werden, da keine definierte Zonenachse parallel zum Elektronenstrahl liegt. Diese Aufnahme illustriert die Problematik bei der Untersuchung der Entwicklung von Texturen im Verlauf des Schichtwachstums anhand von 2-dimensionalen, für die TEM-Analyse präparierten Proben. Es können nur solche Schichtbereiche über die gesamte Filmdicke analysiert werden, bei denen die Ausrichtung überall eine Orientierungsbestimmung zulässt. Wie die Orientierungen in den Bereichen A und B entstanden, sind bleibt unklar, da eine Orientierungsbestimmung unterhalb der markierten Bereiche nicht möglich war. Eine Ausnahme bildet Säule C. Sie wird später einer genaueren Untersuchung unterworfen.

In diesem Abschnitt werden zunächst zwei Mechanismen vorgestellt, die eine Orientierungsänderung vermitteln und so zur Ausbildung der Textur beitragen. Ferner wird die Säulenstruktur näher betrachtet und zum Abschluss auf die Oberseite dicker IBAD-YSZ-Filme eingegangen.

\subsection{Kontinuierliche Orientierungsänderung während des Wachstums}

Abbildung 6.2 zeigt eine hochauflösende Gitterabbildung von IBAD-YSZ auf $\mathrm{Si}_{/} \mathrm{SiO}_{2}$ als Beispiel für einen Filmbereich, in dem die kristallographische Ausrichtung die Beobachtungen einer kontinuierlichen Orientierungsänderung innerhalb von 100 nm Filmdicke zulässt. Man blickt senkrecht zur Projektion unterstützenden Ionenstrahls. Die lokale Orientierung wurden an den markierten Stellen mittels FFT bestimmt. In den ersten ca. $20 \mathrm{~nm}$ ist weder in der Fourier-Transformation noch im Realbild eine kristalline Struktur zu erkennen. Wie die Untersuchungen zum Frühstadium gezeigt haben, enthält dieser Bereich amorphe und kristalline Anteile. Ab einer Schichtdicke von ca. $20 \mathrm{~nm}$ sind Netzebenen im Realbild zu erkennen. Die Auswertung der FFT zeigt, dass man in diesem Bereich entlang der $<110>$-Richtung der YSZ-Einheitszelle sieht. Die Normale der (001)-Ebene ist hier um ca. $30^{\circ}$ gegen die Substratnormalen verkippt. Der Vergleich der einzelnen FFTs zeigt deutlich die Änderung der $<001>$ Richtung mit zunehmender Filmdicke. In einer Filmdicke von ca. $100 \mathrm{~nm}$ hat sich die $<001>$ Richtung parallel zur Substratnormalen eingestellt. In dieser speziellen Wachstumssäule beobachtet man also eine Drehung der $<001>$-Richtung um die $<110>$-Blickrichtungsachse, die 
parallel zum Elektronenstrahl verläuft. Die Projektion des einfallenden Ionenstrahls auf die Substratebene liegt bei diesem Prozess stets parallel zur Projektion der $<\overline{1} 10>$-Richtung des YSZ auf die Substratebene. Es wird also eine $<111>$-Richtung mit zunehmender Filmdicke parallel zum unterstützenden Ionenstrahl ausgerichtet. Ist dies geschehen, erfolgt eine Verbreiterung des Korns. Der weitere Verlauf ist leider nicht mehr zu beobachten, da die Probe an dieser Stelle bereits bis zur Lochentstehung gedünnt wurde.

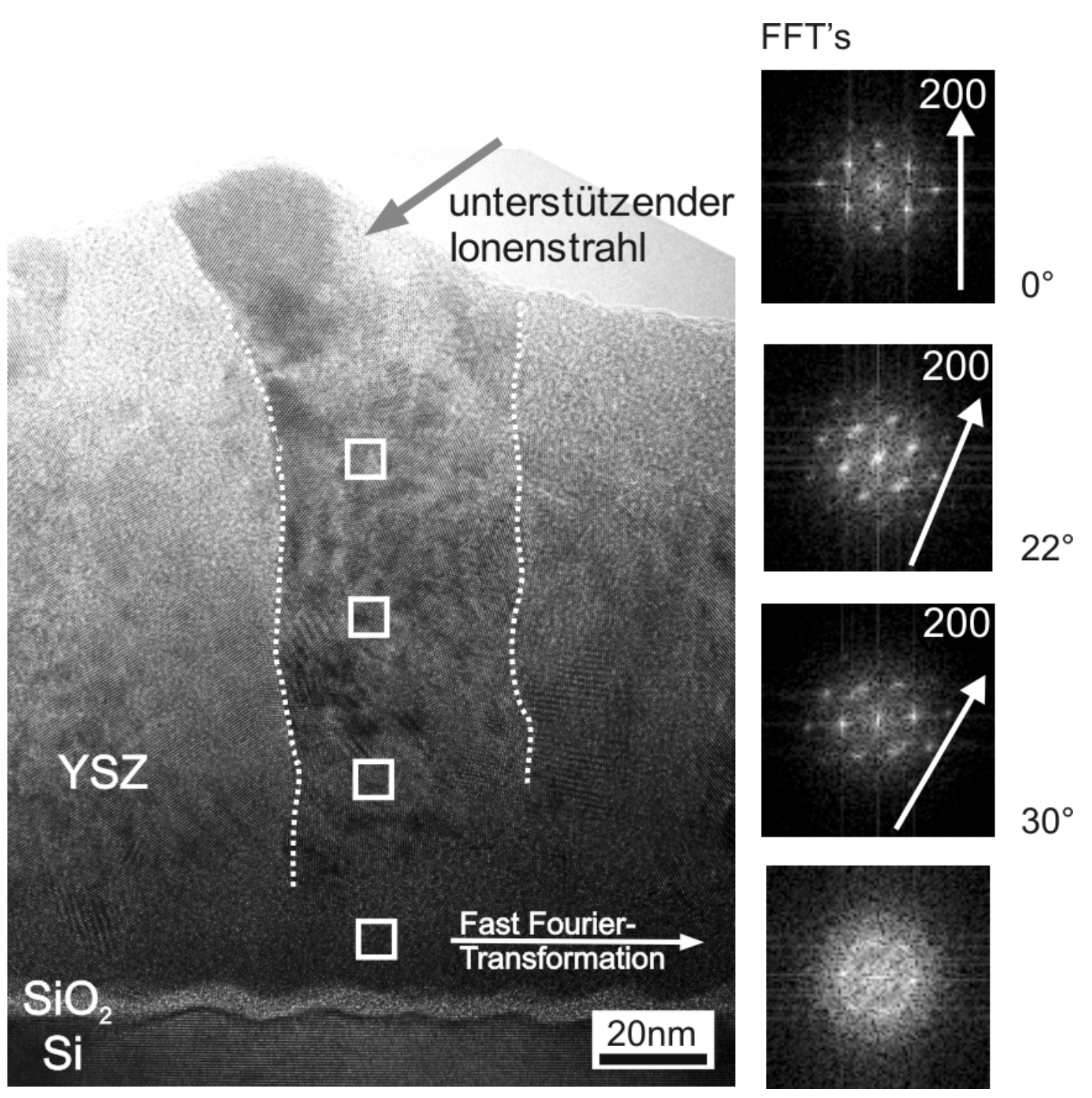

Abbildung 6.2: (001)-Texturverbesserung innerhalb 100 nm Filmwachstum, HREMQuerschnittsaufnahme von IBAD-YSZ auf Silizium, Blick senkrecht zur Projektion des unterstützenden Ionenstrahls. Rechts: FFTs der markierten Bereiche, Pfeile zeigen in <001 >-

Richtung des YSZ. 
Um diese scheinbar kontinuierliche Texturverbesserung auf atomarer Ebene an den Kristallebenen zu verdeutlichen, wurde Abbildung 6.2 in Richtung der Substratnormalen mittels eines Bildbearbeitungsprogramms vertikal gestaucht. Ein $n \times n$ großer Bereich wurde anschließend fouriertransformiert. Aus der erhaltenen Transformation wurden mittels Masken $\{111\}$-Reflexe paarweise ausgeschnitten. So war es möglich, durch Rücktransformation (inverse FFT) eine einzelne $\{111\}$-Ebenenschar abzubilden. Abbildung 6.3 zeigt das Ergebnis dieser Bearbeitung. Es zeigt sich, dass in diesem Fall die Drehung der Netzebenen tatsächlich bis auf die Größenskala einzelner Netzebenen kontinuierlich erfolgt.

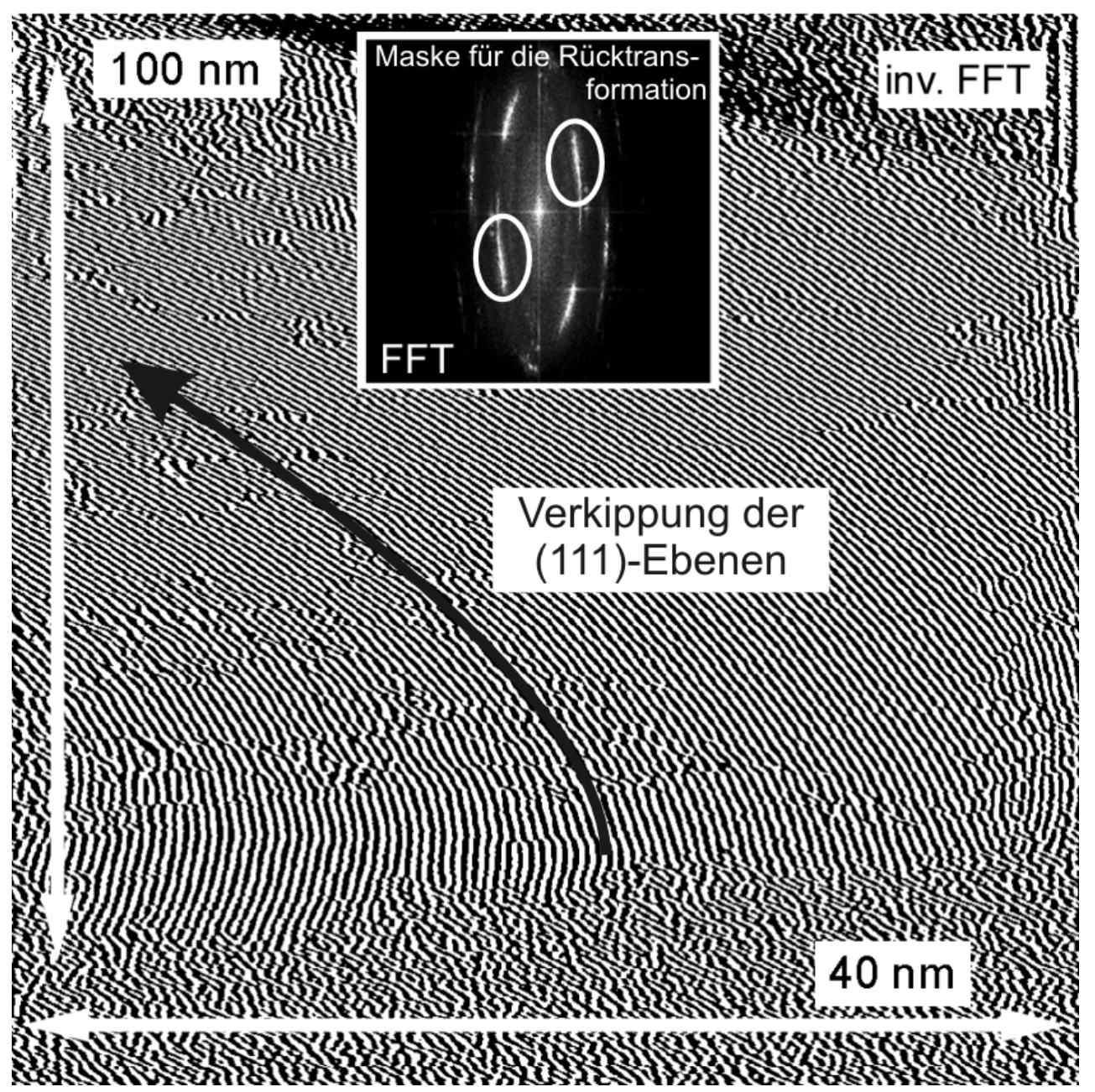

Abbildung 6.3: Eine der \{111\}-Ebenenscharen der gestauchten Abbildung 6.2. Eingefügt ist die dazugehörige FFT, mit Markierung der Maske für die Rücktransformation (inv. FFT). 
Die Änderung der Orientierung der Ebenen ist in Abbildung 6.4 veranschaulicht. Die Grafik zeigt die Änderung der Winkel der beiden $\{111\}$-Ebenen zur Substratnormalen mit zunehmender Filmdicke. Die Drehung der Ebenen lässt sich durch einen exponentiellen Verlauf darstellen, der sich asymptotisch einem Wert von ca. $57^{\circ}$ nähert. Dieses entspricht in etwa dem Einfallswinkel des unterstützenden Ionenstrahls $\left(55^{\circ}\right)$.

Röntgenmessungen zeigen eine ähnliche exponentielle Verbesserung der out-of-planeTextur (vgl. Abbildung 5.1). Der Vergleich der mikroskopischen Messungen (TEM) mit den makroskopischen Messungen (Röntgen) zeigt, dass die Verbesserung der Textur mit zunehmender Filmdicke auf die gleichen Mechanismen, wie sie im TEM beobachtet wurden, zurückzuführen sein könnte.

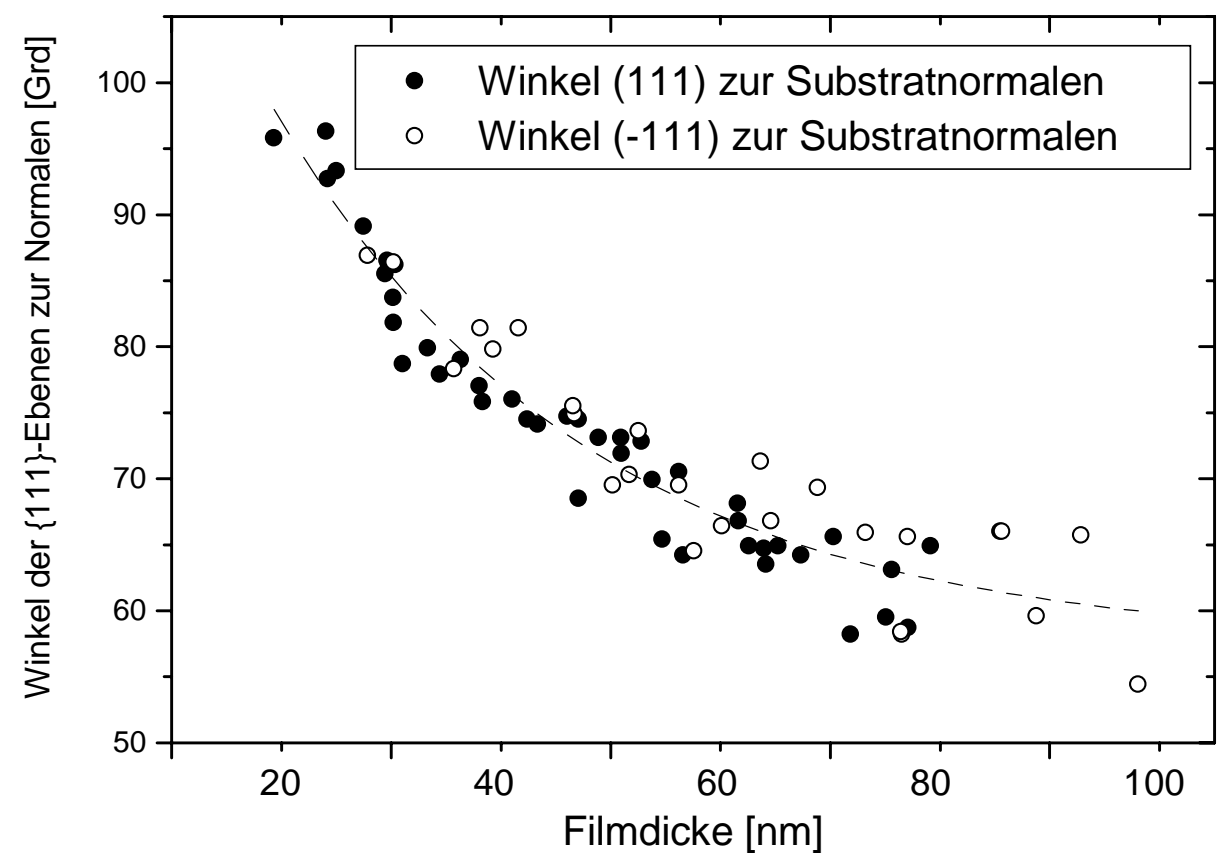

Abbildung 6.4: Winkel der \{111\}-Ebenen zur Substratnormalen der in Abbildung 6.2 gezeigten Wachstumssäule, aufgetragen gegen die Filmdicke. 


\subsection{Defektstrukturen}
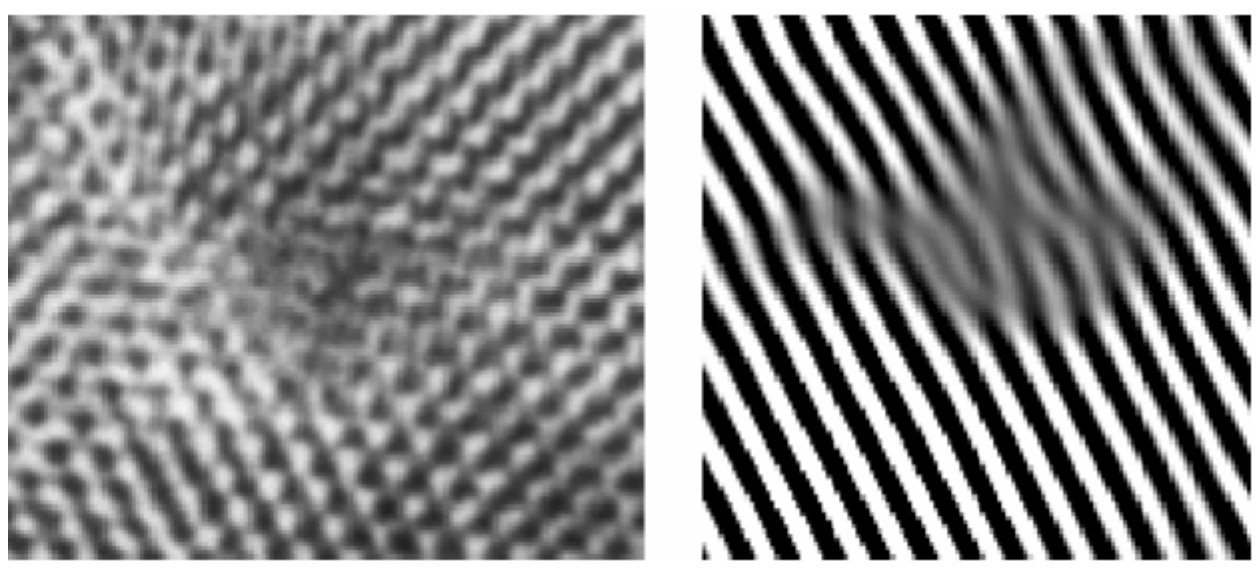

Abbildung 6.5: Darstellung einer defektreichen Probenstelle. Links: Ausschnitt aus einem HREM-Bild, deutlich sind die $\{111\}$-Ebenen zu erkennen. Rechts: inv. FFT einer $\{111\}$ Ebenenschar, man erkennt wie die Ebenen durch den Defekt verzerrt werden.

Versetzungen sind mögliche Defekte, die die oben beschriebenen Verkippungen hervorrufen können, indem zusätzlich eingeschobene Halbebenen in das Kristallgitter eingebracht werden. Sie sind in Hochauflösung jedoch nur dann sichtbar, wenn das Linienelement der Versetzung parallel zum einfallenden Elektronenstrahl verläuft. Dieses ist in der Praxis nicht häufig zu beobachten. In Fourier-Transformationen lassen sich Gitterdefekte wie Versetzungen sichtbar machen, man muss aber jeweils einen Vergleich mit der Probenstelle im Realbild durchführen. Abbildung 6.5 zeigt einen in der hochaufgelösten Aufnahme sichtbaren Defektbereich. Die dazugehörige inverse FFT einer $\{111\}$-Ebenenschar weist auf ein mögliches Vorhandensein einer Versetzung hin. Die in den Proben aufgefundenen, d.h. abbildbaren Versetzungen haben einen Burgersvektor von $a / 2<110>$, das ergaben Burgersumläufe, wie z.B. in Abbildung 6.6 dargestellt. Mit einem Linienelement parallel zum einfallenden Elektronenstrahl handelt es sich somit um Stufenversetzungen. Dieser Versetzungstyp im YSZ wurde auch von anderen Arbeitsgruppen gefunden [Bardal et al., 1994 / Baufeld et al., 1998]. 


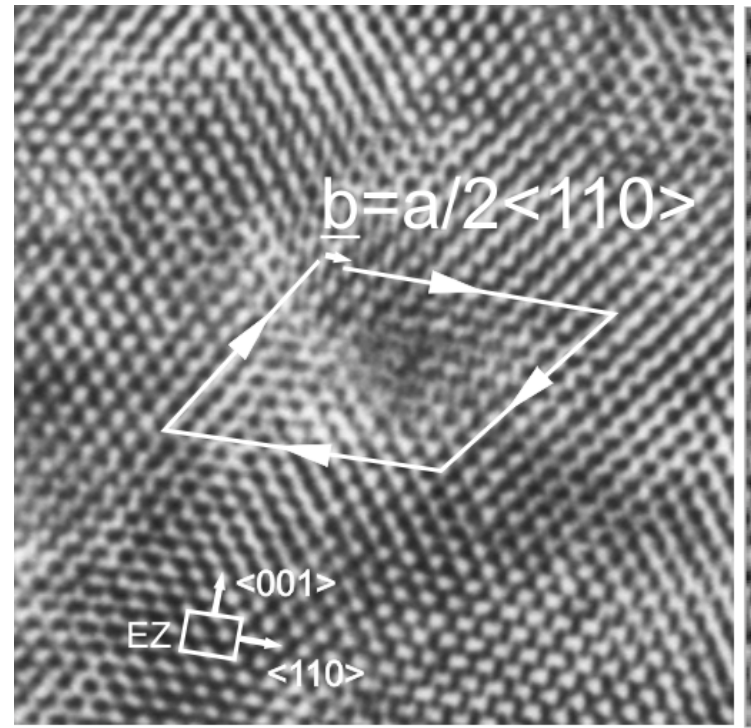

Realbild

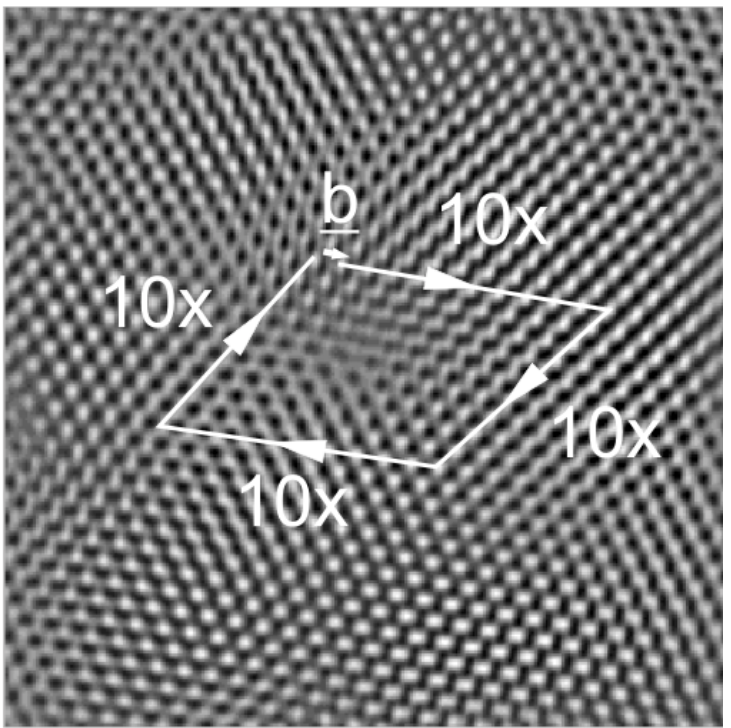

inv. FFT

Abbildung 6.6: Bestimmung des Burgersvektor mittels des Burgerumlaufs im HREM-Bild und im fouriertransformierten Bild, das die $\{111\}$-Ebenen darstellt.

Um die defektreichen Probenstellen vereinfacht darzustellen, wurde das HREM-Bild in Abbildung 6.2 fouriertransformiert. Anschließend wurden die beiden (111) - und (111) -Ebenen herausgefiltert (inv. FFT). Die entstandenen Bilder wurden so bearbeitet, dass helle Stellen defektarme, dunkle dagegen defektreiche Gebiete anzeigen. Das Ergebnis dieser Bearbeitung ist in Abbildung $6.7 \mathrm{zu}$ sehen. Vergleicht man die Bilder, so erkennt man, dass Gebiete, die für die 1111-Darstellung defektarm sind, in der 111 -Darstellung defektreich sind (vgl. „A“ und „B“ in Abbildung 6.7). Die Versetzungsdichte ist im gesamten Bereich homogen und beträgt ca. $10^{12} \mathrm{~cm}^{-2}$, wobei ein leichter Anstieg der Versetzungsdichte an den Säulenrändern zu beobachten ist. 

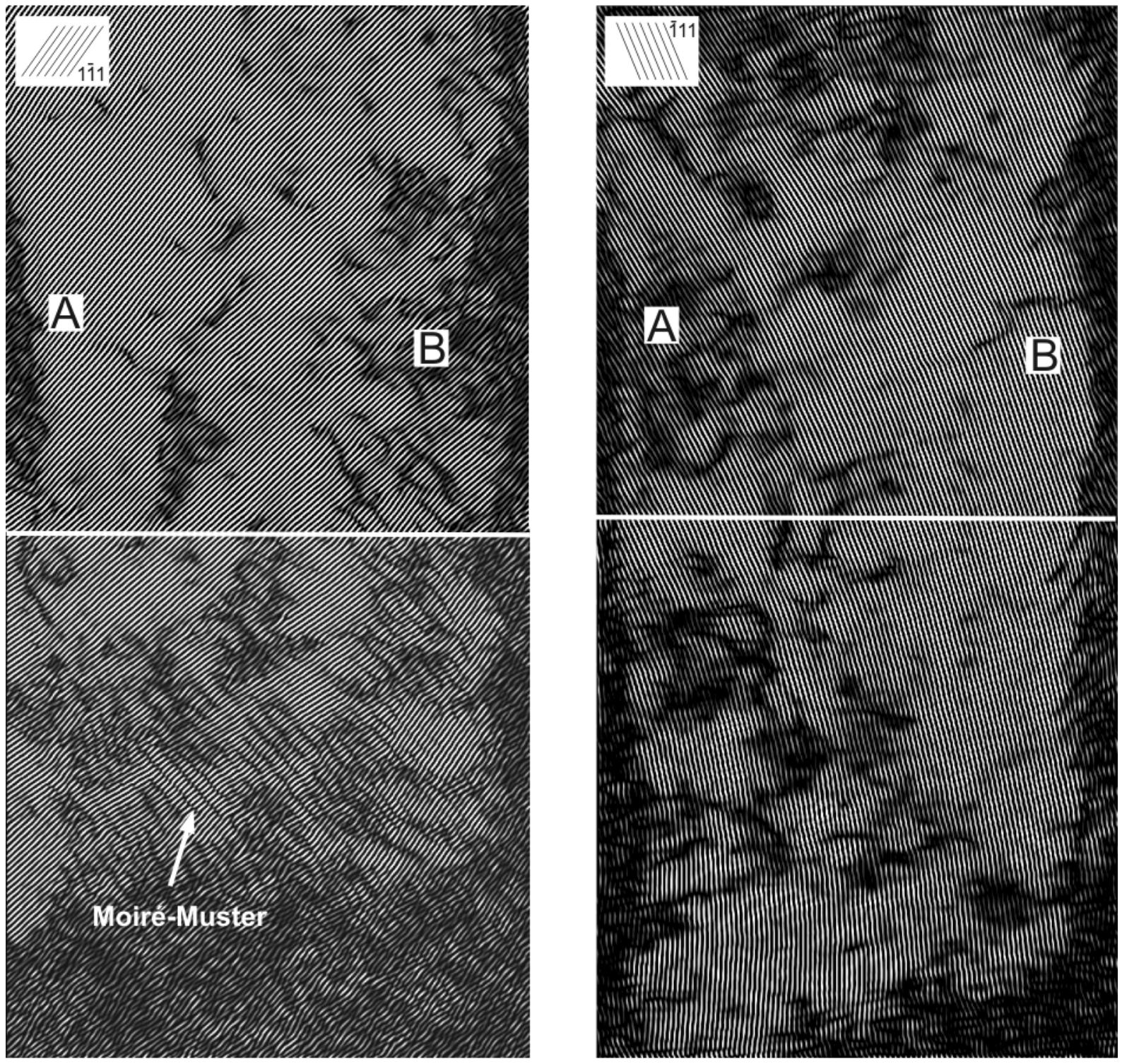

Abbildung 6.7: Inverse FFTs der beiden \{111\}-Ebenenscharen, der in Abbildung 6.2 (Seite 50) dargestellten Wachstumssäule. Dunkle Bereiche entsprechen einer hohen Defektdichte. Im unteren Bereich sind Moiré-Muster zu erkennen, die auf kleine, leicht verdrehte Körner hinweisen. 


\subsection{Diskontinuierliche Orientierungsänderung}

Neben dem quasikontinuierlichen Verkippen von Körnern durch eine weitgehend homogene Versetzungsdichte lassen sich auch unstetige Orientierungsänderungen beobachten. Prinzipiell sind zwei Mechanismen denkbar, die in Wachstumsrichtung im TEM als diskontinuierliche Orientierungsänderung erscheinen lassen. Dies ist zum einen das Wachsen eines andersorientierten Kornes auf einem Korn unter Ausbildung einer kohärenten oder inkohärenten Grenzfläche, zum anderen das Überwachsen durch ein benachbartes Korn. So gibt z.B. Dzick an, dass der Übergang von einem (110)- zu einem (001)-orientierten Wachstum durch eine epitaktische Beziehung zwischen (110)- und (001)-Ebenen vermittelt wird [Dzick, 2000]. Die hier vorgestellten Untersuchungen ergeben für diese Vorstellung zwar keine unterstützenden Hinweise, aber gerade in den Frühstadien des Filmwachstums scheint das Aufeinanderwachsen unterschiedlicher Orientierungen häufig aufzutreten.

Abbildung 6.8 zeigt in einer hochaufgelösten TEM-Querschnittsaufnahme einen kristallinen Bereich, der nach ca. $8 \mathrm{~nm}$ Filmdicke seine relative Orientierung zum IBAD-Strahl durch leichte Verkippung und Verdrehung geringfügig ändert. Im Bild sind die (111)- und (200)Ebenen eingezeichnet. Beide Körner werden durch eine Kleinwinkelkorngrenze getrennt. Es ist hier nicht vollständig auszuschließen, dass eine Wachstumskonkurrenz zweier hintereinanderliegender Körner abgebildet wurde, aber das fehlende (mögliche) Moiré-Muster und die parallel zur Substratebene verlaufende Grenzfläche lassen ein Überwachsen als unwahrscheinlich erscheinen. Zudem haben alle Untersuchungen zum Frühstadium des Wachstums gezeigt, dass in dieser Phase keine ausgeprägte Wachstumsselektion stattfindet. Diese Wachstumsselektion wäre aber eine Voraussetzung dafür, dass sich schon nach wenigen Nanometer Filmdicke Körner überwachsen. 


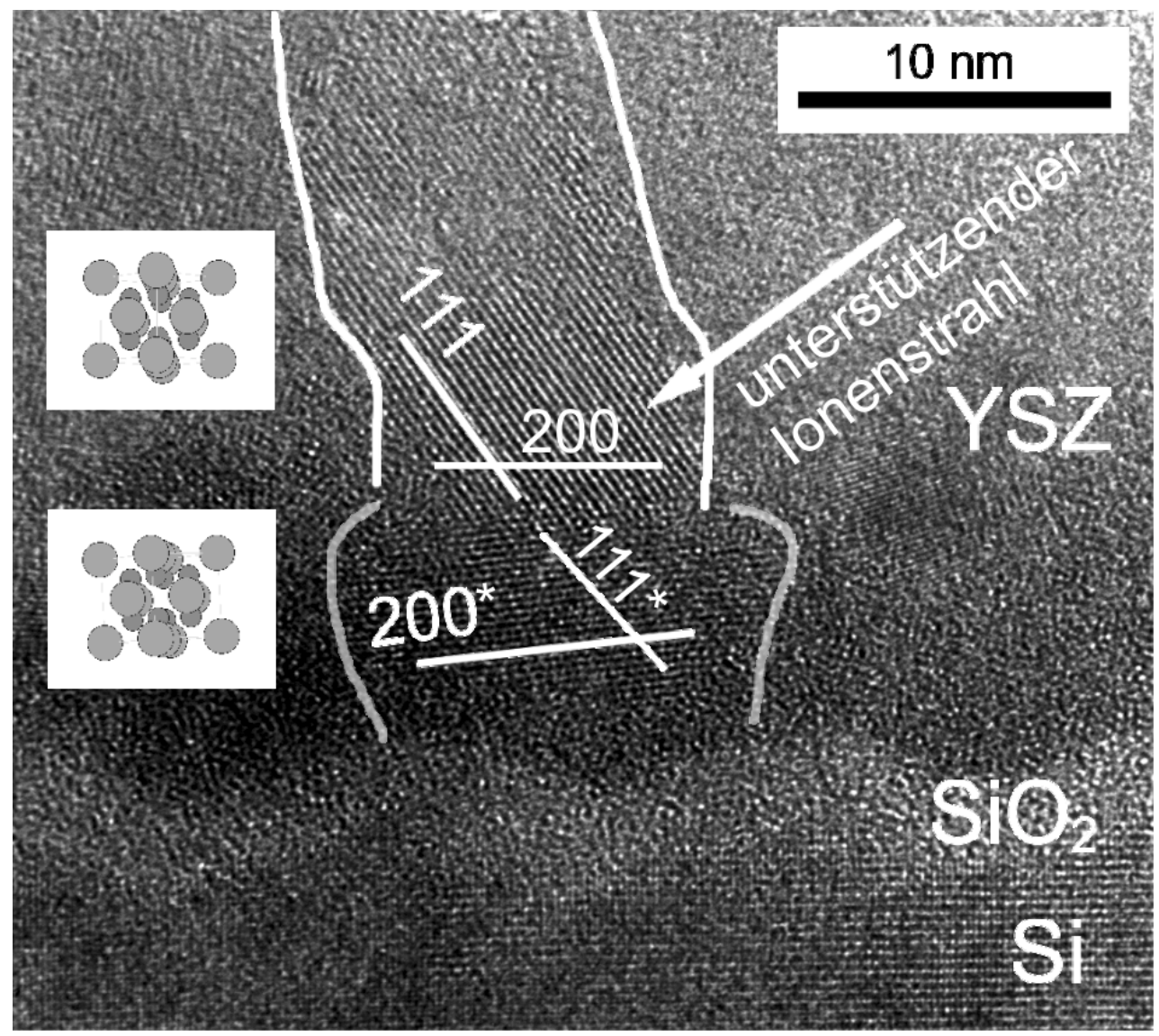

Abbildung 6.8: IBAD-YSZ auf Silizium, HREM-Querschnittsaufnahme, Blick senkrecht zur Projektion des unterstützenden Ionenstrahls. Ein kristalliner Bereich ändert seine Orientierung durch neues Ankeimen. Die kleinen Bilder zeigen YSZ-Einheitszellen, die so orientiert sind, wie die jeweiligen Bereiche im TEM-Bild.

Neben der kontinuierlichen Orientierungsänderung und dem Ankeimen neu-orientierter Körner ist das Überwachsen ein häufig beobachteter Prozess, der sich in Filmdicken von $>100 \mathrm{~nm}$ abspielt. Abbildung 6.9 zeigt eine hochaufgelöste Querschnittsaufnahme eines IBAD-YSZ-Films in einer Filmdicke von ca. $200 \mathrm{~nm}$. Der untere, (110)-orientierte Bereich wird von einem Bereich überwachsen, dessen (001)-Orientierung um ca. $20^{\circ}$ aus der Substratebene verkippt ist. Die durchgezogene Linie markiert dabei den Verlauf der Korngrenze. Zwar gelten auch hier prinzipiell die gleichen Vorbehalte wie in Abbildung 6.8, die stark gekrümmte Grenzfläche legt aber ein Überwachsprozess nahe. Während sich der (001)-Bereich lateral verbreitert, ist im oberen Bereich der Abbildung 6.9 die Änderung des (001)-Orientierung zu erkennen (gestrichelte Linie). 


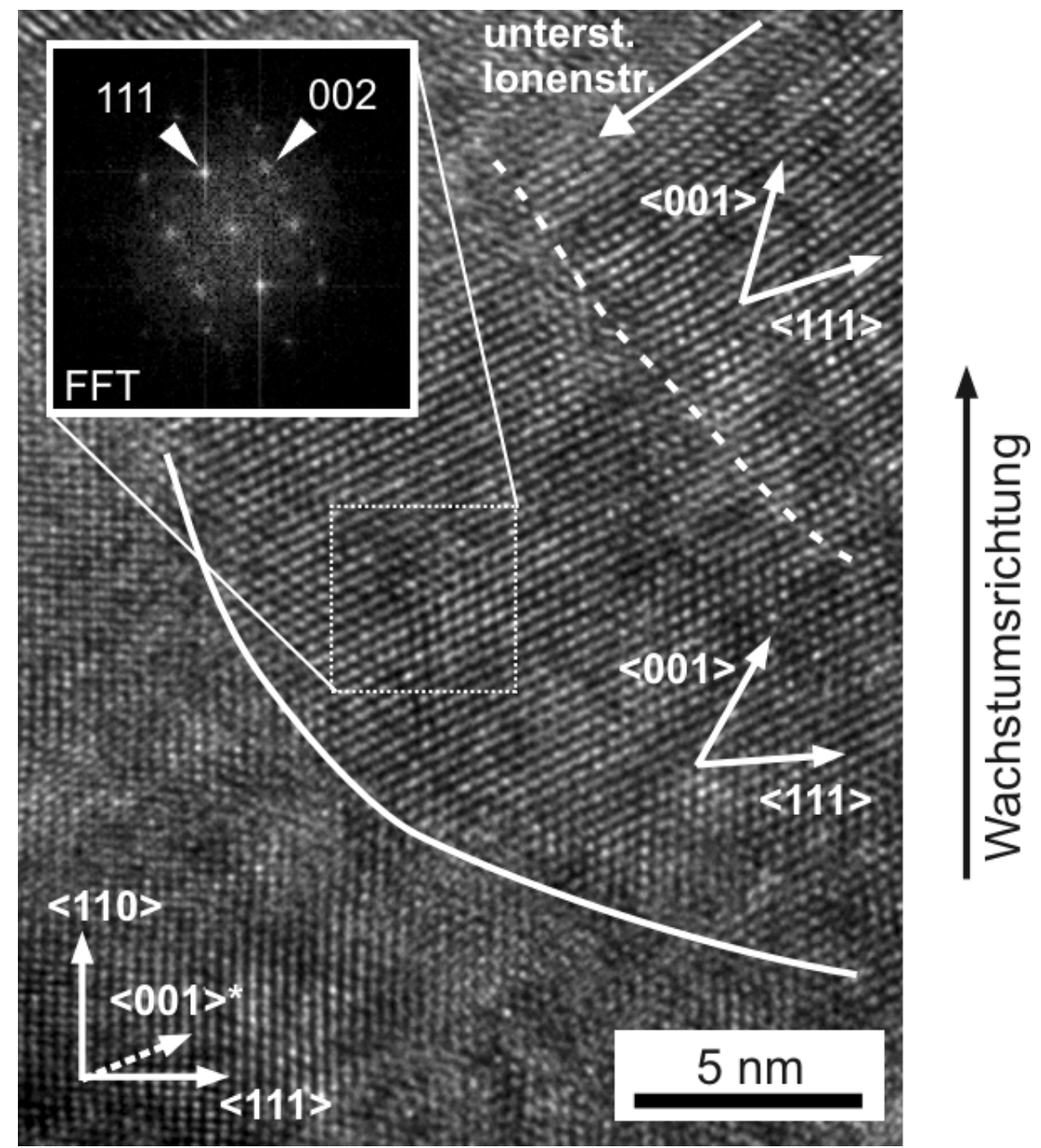

Abbildung 6.9: HREM-Netzebenenabbildung, IBAD-YSZ im Querschnitt. Die Aufnahme zeigt das Überwachsen eines (110)-orientierten Bereichs von einem (001)-orientierten in einer Schichtdicke von ca. $200 \mathrm{~nm}$. Innerhalb von ca. $15 \mathrm{~nm}$ Schichtwachstum verbessert sich dann zusätzlich die (001)-Orientierung.

Zusammengefasst sind also beide Mechanismen der diskontinuierliche Orientierungsänderung wahrscheinlich möglich, wobei das Aufeinanderwachsen eher im Frühstadium, das Überwachsen im späteren Stadium dominiert.

\subsection{Säulenwachstum}

Im vorangehenden Abschnitt wurde gezeigt, dass sich in den YSZ-Filmen zunächst eine (001)-out-of-plane-Textur einstellt. Im weiteren Verlauf des Filmwachstums entwickelt sich dann eine Ausrichtung in der Ebene. Dieses wird im folgenden genauer untersucht.

Nach einer Schichtdicke von ca. 100 - 200 nm existieren überwiegend (001)-texturierte Domänen, die ein kolumnares Wachstum zeigen. Im Gegensatz zu einer Drahttextur besitzen 
diese Domänen jedoch eine Vorzugsorientierung in der Filmebene, das zeigen röntgenographische Untersuchungen dünner Schichten.

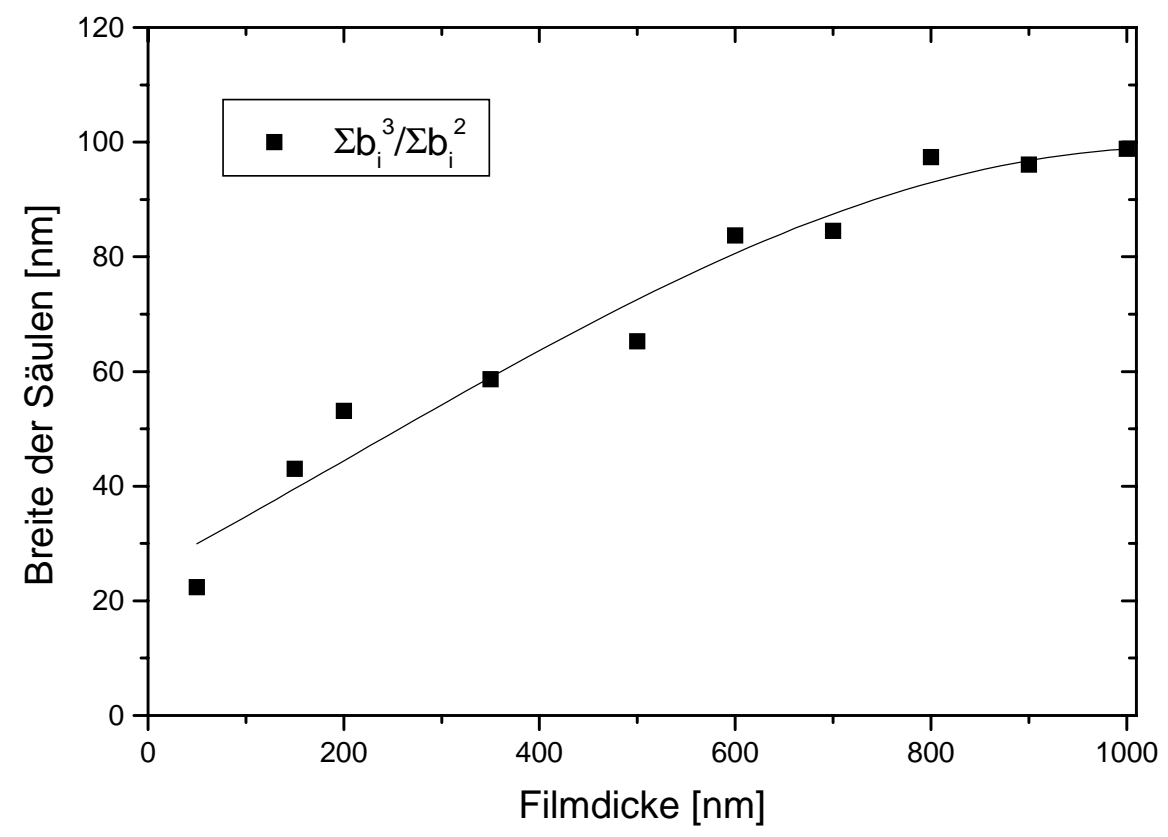

Abbildung 6.10: Säulenverbreiterung in Abhängigkeit der YSZ-Filmdicke. Die eingezeichnete Kurve dient nur zur Veranschaulichung.

In Abbildung 6.10 ist die Säulenverbreiterung in Abhängigkeit von der Filmdicke dargestellt. Dazu wurden in Querschnittsbildern die Breiten $b_{i}$ der Bereiche gleichen Kontrasts gemessen. Die Werte wurden mit dem relativen Flächenanteil $b_{i}^{2}$, der als quadratisch angenommenen Körner, gewichtet. Der Fehler ist bei der Bestimmung der Breite in einer Filmdicke bis zu ca. $300 \mathrm{~nm}$ relativ hoch, da in diesem Bereich viele Säulen bzw. Körner existieren. Ausgehend von einer Korngröße von ca. $30 \mathrm{~nm}$ bei den ersten sich abzeichnenden Körnern, steigt der gemittelte Korndurchmesser zunächst fast linear an. Die Breite der Säulen in einer Schichtdicke von $200-300 \mathrm{~nm}$ beträgt $30-70 \mathrm{~nm}$. Ab einer Filmdicke von ca. $500-600 \mathrm{~nm}$ ist nur noch eine geringe Verbreiterung der Säulen mit zunehmender Filmdicke zu beobachten. Bei Filmdicken von $1-1,3 \mu \mathrm{m}$ haben sich viele Säulen bis zu einer durchschnittlichen Größe von $100 \mathrm{~nm}$ verbreitert. Allerdings existieren in diesem Filmdickenbereich aber auch Säulen, die nur eine Breite von ca. $30 \mathrm{~nm}$ bzw. eine Breite von bis zu $150 \mathrm{~nm}$ aufweisen. Ab einer Filmdicke von ca. $500 \mathrm{~nm}$ verlaufen die Säulengrenzen jedoch schon praktisch parallel zur Wachstumsrichtung. 


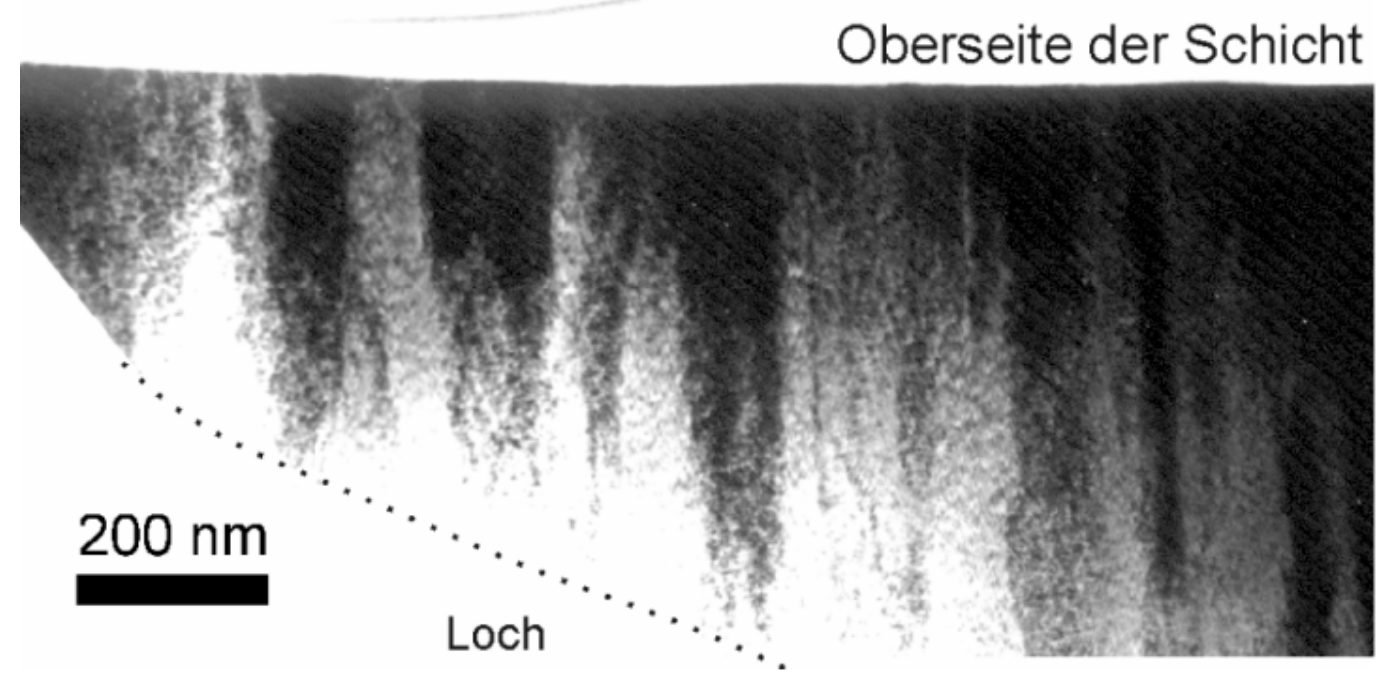

Abbildung 6.11: TEM-Querschnittsaufnahme der Oberseite einer ca. 1,3 $\mu$ m dicken IBADYSZ-Schicht. Dunkle Bereiche sind so orientiert, dass die $\langle 110>-Z$ Znenachsen nahezu parallel zum Elektronenstrahl verlaufen. Die Verbreiterung bzw. Verjüngung der Säulen in der Nähe des Lochrandes ist auf die Keilförmige Form der Probe zurückzuführen.

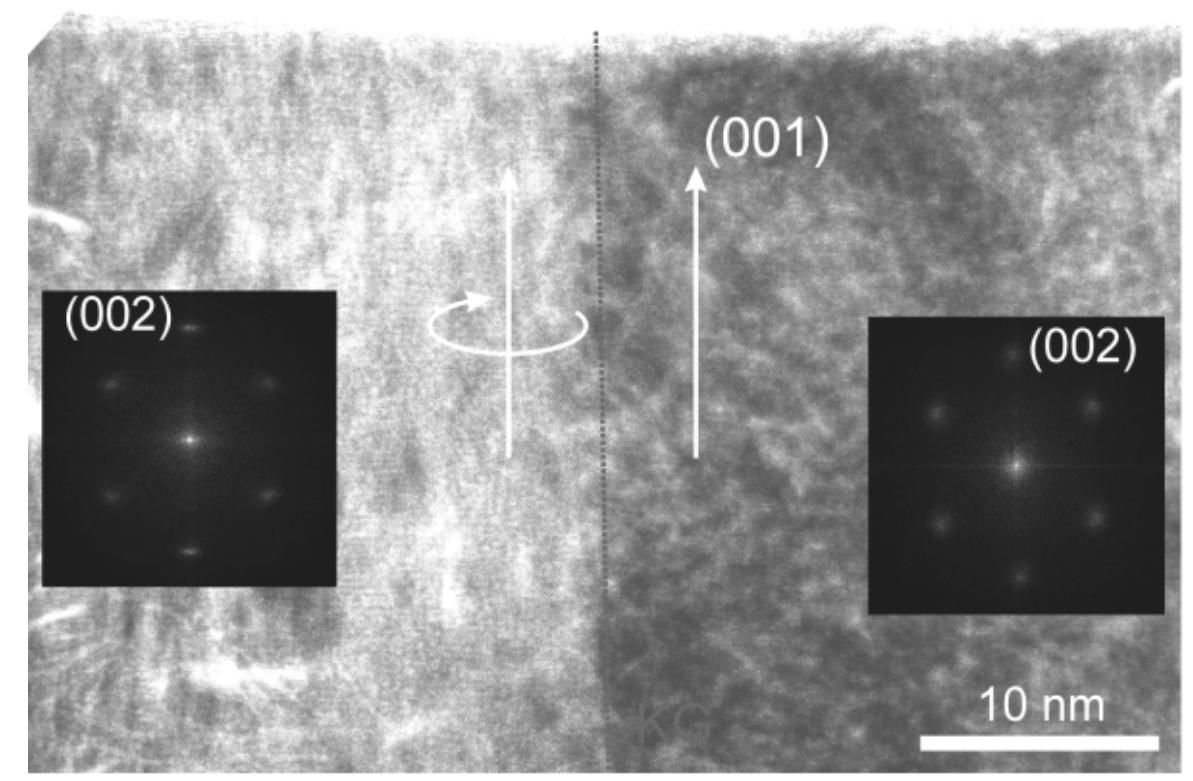

Abbildung 6.12: HREM-Querschnittsaufnahme der Oberseite der YSZ-Schicht. Der Kontrastunterschied der beiden (001)-Säulen beruht auf einer in-plane-Drehung um wenige Grad (s. Pfeil).

Abbildung 6.11 und Abbildung 6.12 zeigen ein Übersichtsbild bzw. eine Detailaufnahme der Oberseite eines ca. 1,3 $\mu \mathrm{m}$ dicken IBAD-YSZ-Films im Querschnitt. Wie schon in Abschnitt 5.4 gezeigt, unterscheiden sich in diesem Filmdickenbereich die (001)-Orientierungen 
benachbarter Säulen nur um wenige Grad. So sind die out-of-plane-Orientierungen der in Abbildung $6.12 \mathrm{zu}$ sehenden Säulen annähernd parallel (vgl. FFTs). Die Kontrastunterschiede in diesem Bild sind allein auf verschiedene in-plane-Orientierungen der Säulen zurückzuführen.

Im folgenden soll eine Grenze zwischen zwei Wachstumssäulen näher betrachtet werden.

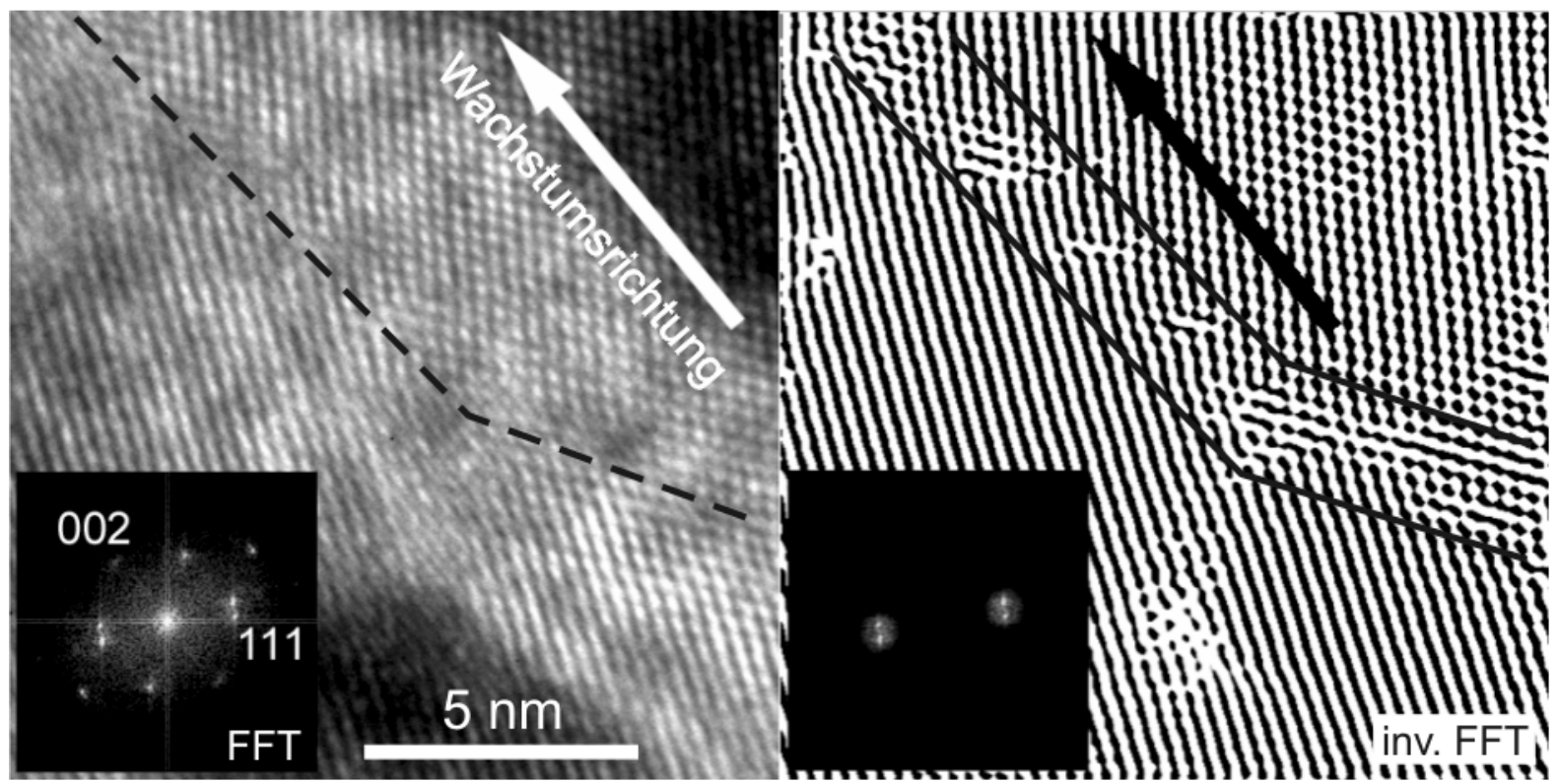

Abbildung 6.13: Korngrenze zweier (001)-orientierter Bereiche, die parallel zur Wachstumsrichtung verläuft. Links: HREM-Aufnahme von IBAD-YSZ im Querschnitt, Schichtdicke ca. $500 \mathrm{~nm}$. Rechts: inverse FFT, abgebildet ist eine (111)-Ebenenschar. Die Pfeile zeigen in Wachstumsrichtung.

Abbildung 6.13 zeigt in einem hochaufgelösten Bild die Grenze zweier Säulen in einer Filmdicke von ca. $400 \mathrm{~nm}$ im Querschnitt. Eine Korngrenze trennt zwei (001)-orientierte Säulen. Um den Verlauf der Grenze deutlicher hervorzuheben, wurde mittels inverser FourierTransformation nur eine $\{111\}$-Ebenenschar abgebildet. Die Korngrenze zeigt einen Verlauf parallel zur Wachstumsrichtung, wobei die gestörten Bereiche in der inversen FFT auf Versetzungen hinweisen, die regelmäßig in einem Abstand von ca. 2,5 nm angeordnet sind. Die gestörte Zone erstreckt sich nur über wenige Atomlagen. Eine Aufspaltung aller Beugungsreflexe würde auf eine reine Kippgrenze hinweisen. Da jedoch nur ein Beugungsreflex eine Aufspaltung vorweist, hat die Korngrenze zusätzlich einen Drehanteil. Misst man die Winkel der (111)-Ebenen so erhält man einen Kippanteil in der Bildebene von ca. $12^{\circ}$. Über die Verkippung senkrecht dazu können keine Aussagen gemacht werden. Es reichen allerdings wenige Grad Verkippung aus, um Netzebenen im TEM-Bild nicht mehr darstellen zu können. 
An dieser Stelle sei auf eine besondere Eigenschaft benachbarter Säulen hingewiesen. So ist in jedem der untersuchten YSZ-Filmen beobachtet worden, dass benachbarte Säulen häufig entgegengesetzten Kontrast haben. Diese Ergebnis lässt sich auch auf TEM-Aufnahmen anderer Arbeitsgruppen ausweiten [Iijima et al., 1998a / Wiesmann, 1998]. Möglicherweise handelt es sich hierbei um eine spannungsinduzierte Orientierungsänderung in der Filmebene, ähnlich einer Verzwillingung.

\subsection{Oberseite des IBAD-YSZ-Films}

Zum Abschluss dieses Abschnitts soll die Filmoberseite ca. $1 \mu \mathrm{m}$ dicker YSZ-Filme näher betrachtet werden. Die Textur und die Mikrostruktur in diesem Bereich bestimmt im wesentlichen die Eigenschaften epitaktisch abgeschiedener HTSL-Filme.

Die HREM-Abbildung 6.14 zeigt einen IBAD-YSZ-Film, der so präpariert wurde, dass der Bereich nahe der Oberseite in Aufsicht zu sehen ist. Anhand der Netzebenen kann mittels der FFT die lokale Orientierung bestimmt werden. Die Auswertung des Beugungsmuster ergibt eine (001)-out-of-plane-Orientierung. Üblicherweise zeigt der Film keine deutlichen Kontrastunterschiede in Aufsicht, was auf eine ausgeprägte Textur hinweist.

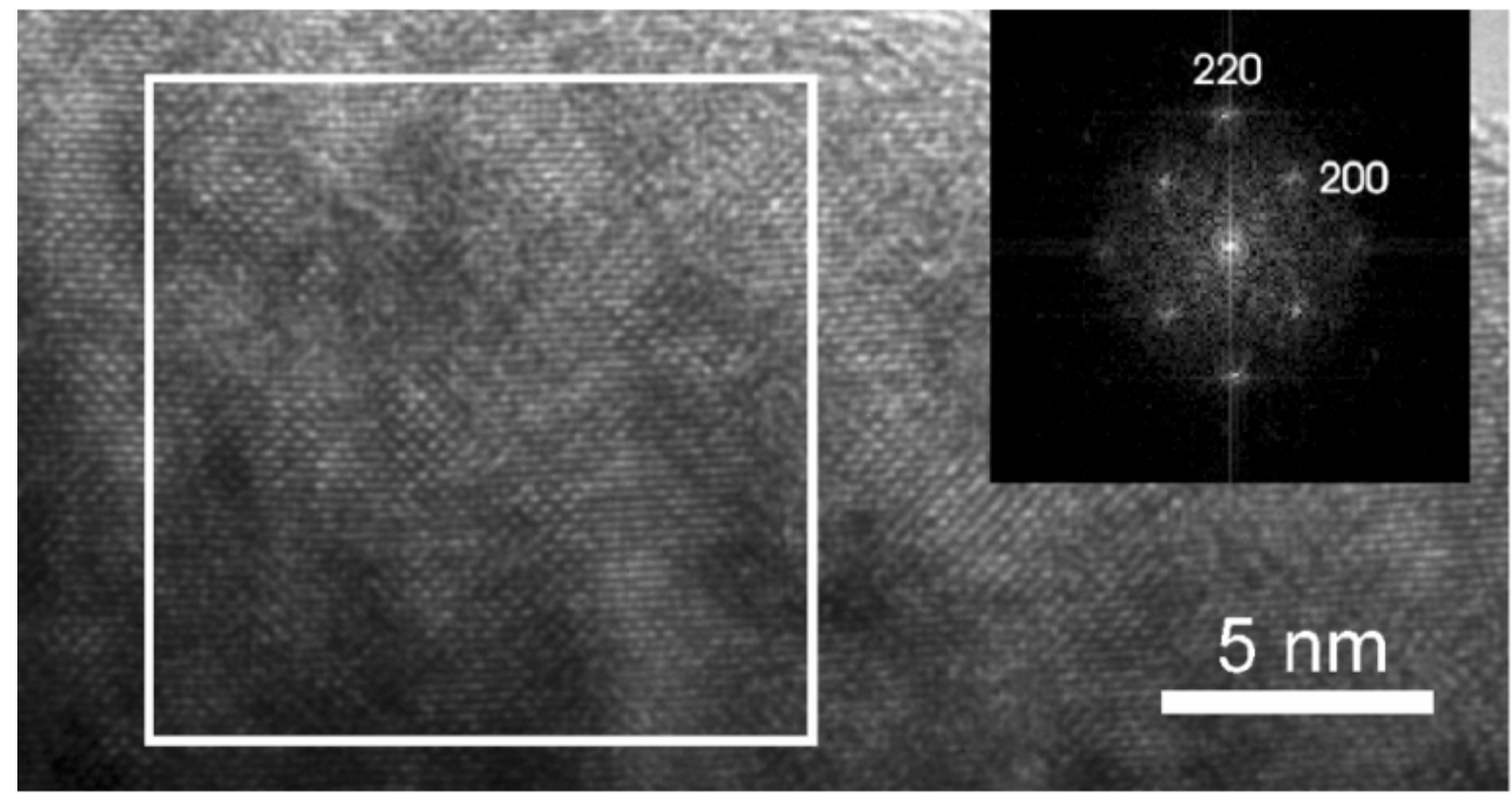

Abbildung 6.14: Hochaufgelöste TEM-Aufnahme nahe der Filmoberseite in Aufsicht. Die FFT zeigt die (001)-Orientierung. 


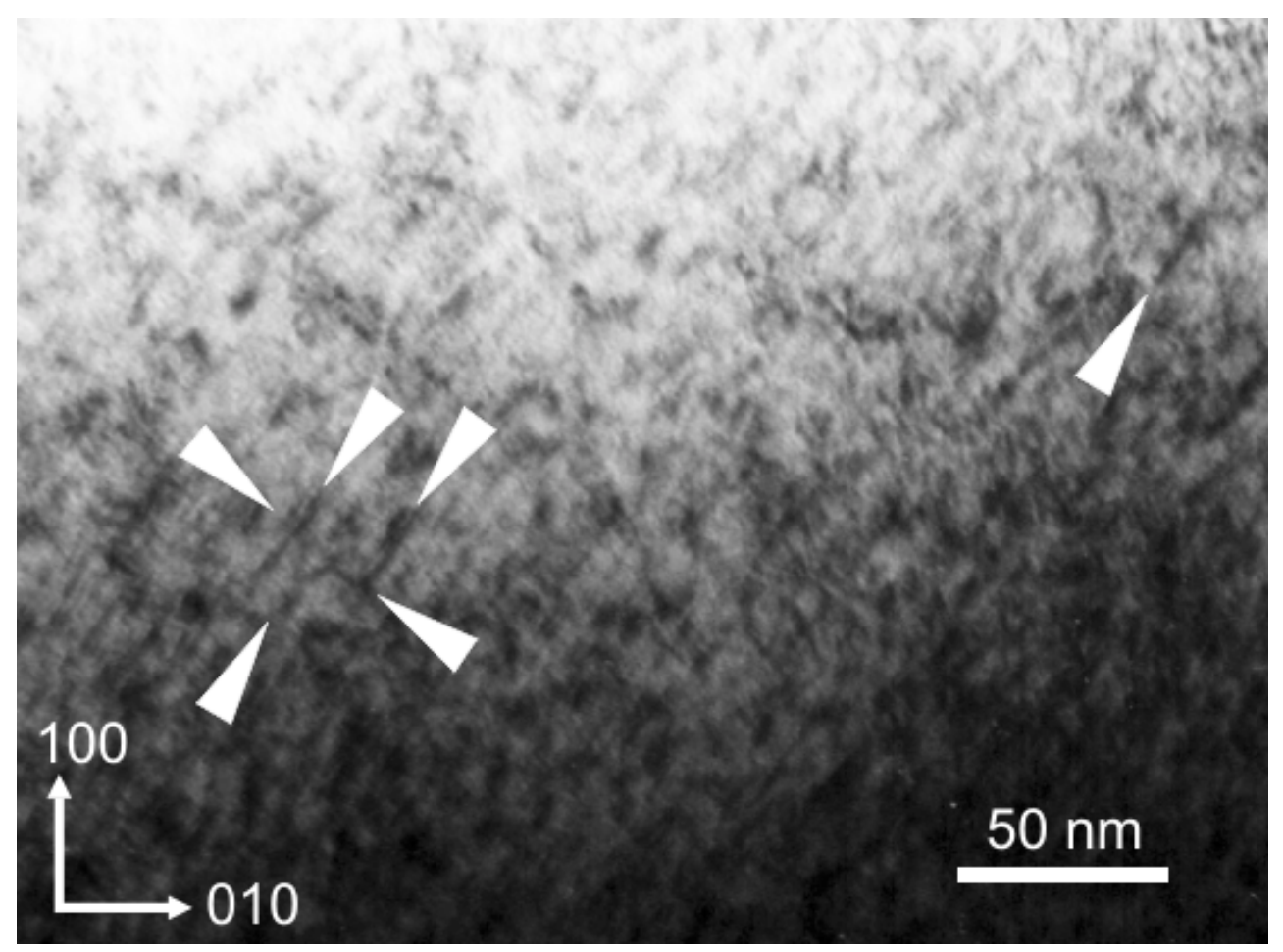

Abbildung 6.15: IBAD-YSZ in Aufsicht, Blick in Richtung der Filmnormalen. Die Probe wurde so gedünnt, dass man den Bereich nahe der Oberseite eines ca. 1,2 $\mu$ m dicken Films sieht. Die Pfeile markieren Versetzungen.

Abbildung 6.15 zeigt einen Bereich nahe der Oberseite eines ca. 1,2 $\mu \mathrm{m}$ dicke IBAD-YSZFilms in Aufsicht. Neben dem nach oben heller werdenden Kontrast (Keilform der Probe), sind zusätzlich Spannungskontraste zu sehen. Sie werden durch senkrecht aufeinanderstehenden Versetzungen verursacht, die in Abbildung 6.15 mit Pfeilen gekennzeichnet sind. Die Versetzungsdichte beträgt ca. $10^{11} \mathrm{~cm}^{-2}$ und ist damit um einen Faktor 10 geringer als die in Abschnitt 6.2 im früheren Wachstumsstadium bestimmten. Da der Film keine reine Einkristallinität vorweist, ist es nicht möglich, mit Hilfe von KIKUCHI-Linien Defekte, wie z.B. Versetzungen definiert sichtbar zu machen, um so z.B. den Burgersvektor zu bestimmen (siehe z.B. [Williams und Carter, 1996]. In Abschnitt 5.4 wurde der Burgersvektor mittels Burgersvektorumläufe auf $\underline{b}=a / 2<110>$ bestimmt. Im Falle, dass die hier abgebildeten Versetzungen diesen Burgersvektor haben, und die Linienelemente senkrecht zum Burgersvektor stehen, handelt es sich um reine Stufenversetzungen. Burgersvektor und Gleitebene $(\underline{b} \times \mathrm{d} \underline{s})$ ergeben somit ein Gleitsystem von $\{100\} a / 2<110>$. Dieses Ergebnis stimmt mit Versetzungsuntersuchungen mittels TEM an verformten YSZ-Einkristallen überein [B. Baufeld et al., 1998].

Zusätzlich lassen sich aber auch an der Oberseite ca. $1 \mu \mathrm{m}$ dicker YSZ-Filme Bereiche mit deutlich gestörteren Kristallstrukturen beobachten. Beim ionenstrahlunterstütztem Wachstum stellt sich also trotz sich verbessernder Textur kein quasi-einkristalliner Zustand ein. 
Abbildung 6.16 zeigt exemplarisch zwei Feinbereichsbeugungsbilder. Sie stammen jeweils von Bereichen mit ca. $500 \mathrm{~nm}$ Durchmesser. Dabei ist das Feinbereichsbeugungsbild in Abbildung 6.16 a) mit der Fast-Fourier-Transformation in Abbildung $6.14 \mathrm{zu}$ vergleichen. Es zeigt nur eine geringe Breite der Häufungspunkte (ca. $5^{\circ}$ ), was auf eine ausgeprägte in-planeTextur hinweist. Dagegen zeigt das Beugungsbild in Abbildung 6.16 b) die Reflexe eines deutlich gestörteren Bereiches. Die Breite der Häufungspunkte beträgt hier ca. $20^{\circ}$. Das dazugehörige Realbild dieses stark gestörten Bereichs ist in Abbildung $6.17 \mathrm{zu}$ sehen. Neben der schlechten in-plane-Textur deuten die kontrastreichen Bereiche in Abbildung 6.17 auf Verkippungen der Körner um einige Grad aus der Filmebene hin. Solche gestörten Bereiche wurden relativ selten in den Proben gefunden.
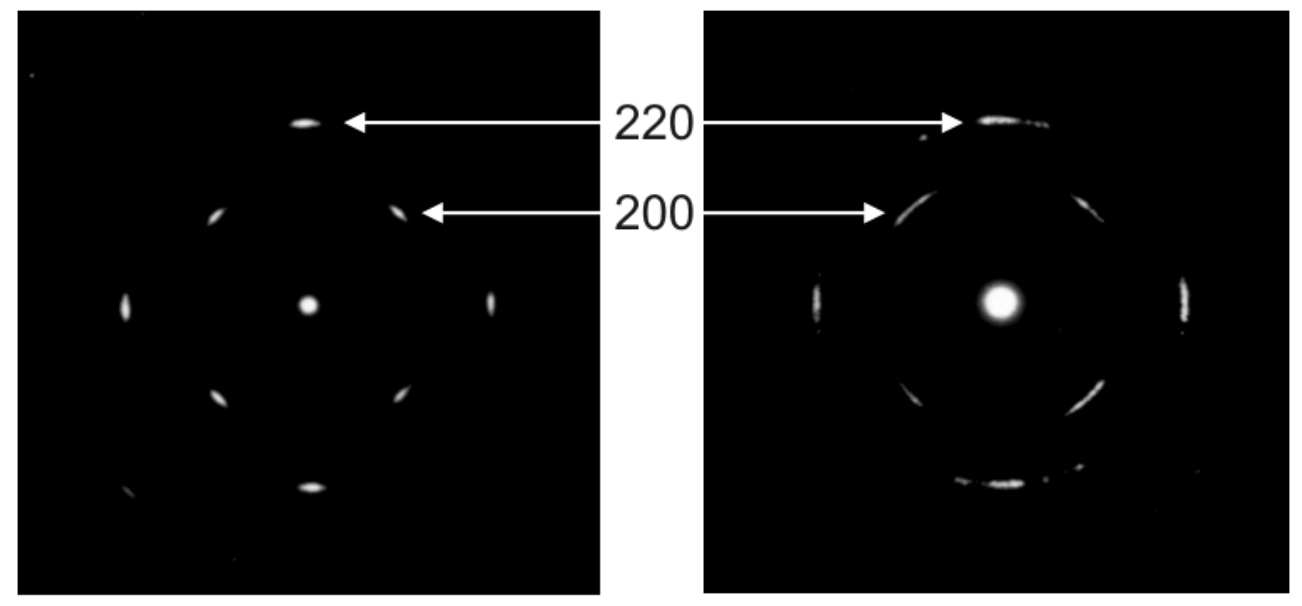

Abbildung 6.16: SAD-Beugungsbilder der Oberseite von IBAD-YSZ in Aufsicht. SADBlendendurchmesser ca. $500 \mathrm{~nm}$. Links: Beugungsbild eines ungestörten Bereichs, rechts: Beugungsbild eines gestörten Bereichs (vgl. Abbildung 6.17). 


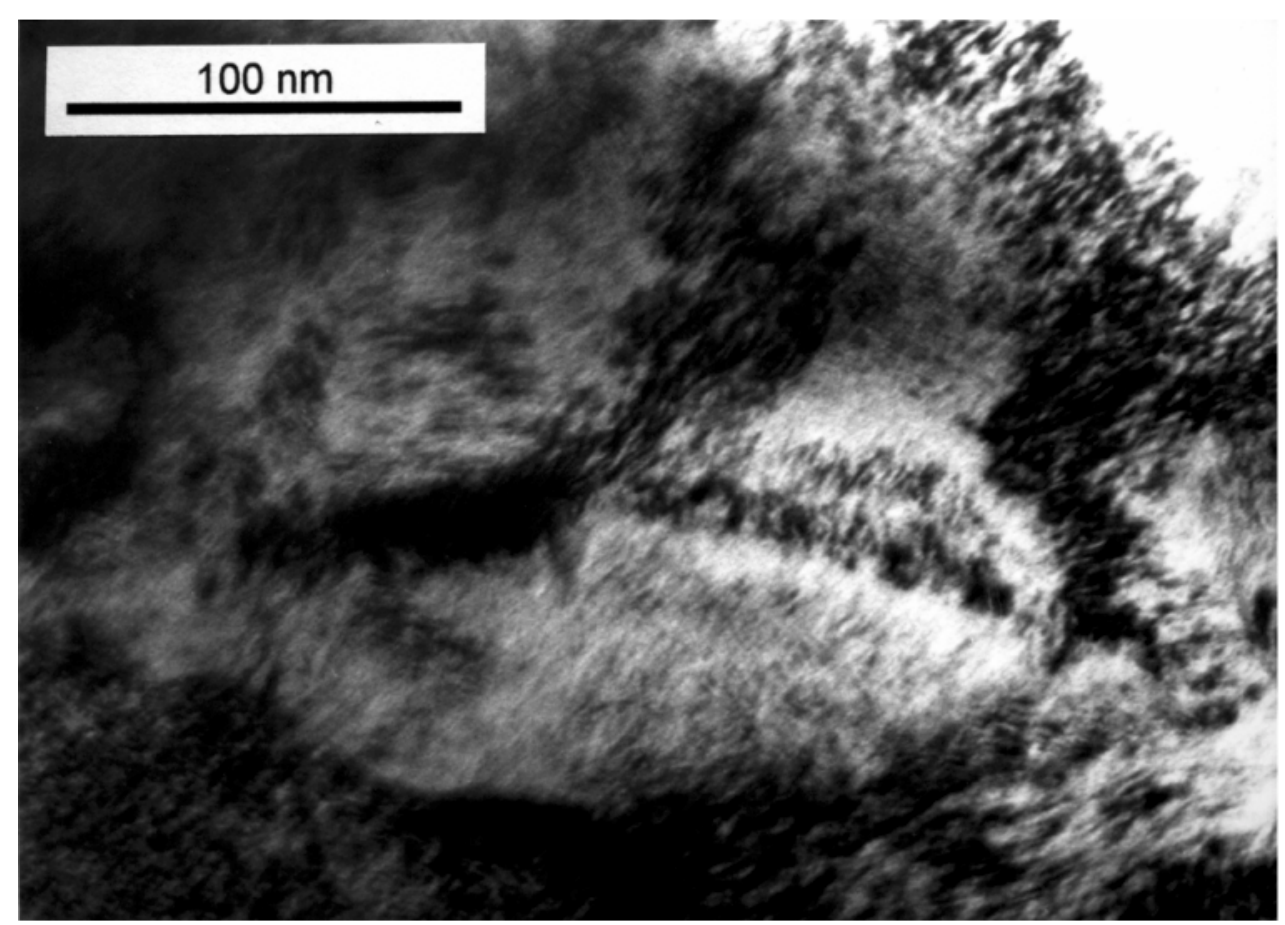

Abbildung 6.17: TEM-Aufnahme von IBAD-YSZ in Aufsicht, gestörter Bereich. Das dazugehörende Beugungsbild ist in Abbildung 6.16 b) zu sehen.

Die TEM-Untersuchungen nahe der Filmoberseite zeigen zusammenfassend eine über große Bereiche stark ausgeprägte out-of-plane-Textur, daneben existieren selten auch sehr gestörte Bereiche. Die Versetzungsdichte beträgt nahe der Filmoberseite ca. $1 \mu \mathrm{m}$ dicker YSZ-Filme ca. $10^{11} \mathrm{~cm}^{-2}$. 
Im folgenden werden zwei TEM-Experimente gezeigt, mittels derer die in-planeOrientierungsunterschiede zwischen den einzelnen Säulen nahe der Filmoberfläche bestimmt werden können. So wurden z.B. Querschnittsproben um die Substratnormale verkippt. Der Verkippungswinkel kann am Goniometer des Probenhalters abgelesen werden. Dabei sollten Bereiche des Films, die anfangs dunklen Kontrast zeigen (nahe der [110]-Zonenachse) während der Verkippung um einige Grad eine Kontrastumkehr zeigen und umgekehrt. Eine Bilderserie dieser Untersuchung zeigt Abbildung 6.18.

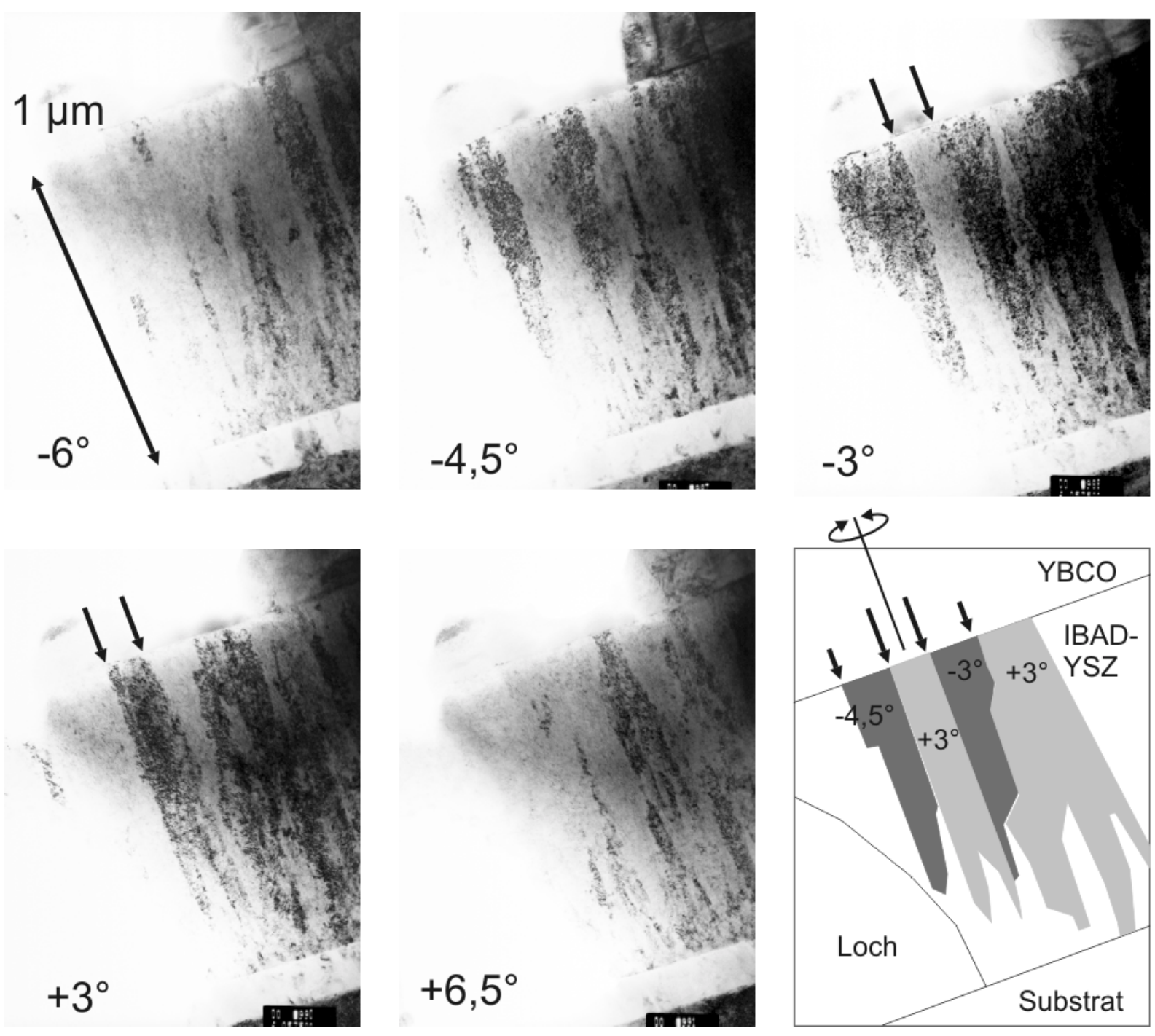

Abbildung 6.18: Verkippungsexperiment im TEM. Der IBAD-YSZ-Film ist im Querschnitt zu sehen und wurde um die Substratachse verkippt. Der jeweilige Verkippungswinkel ist in den Bildern angegeben. Die Pfeile markieren exemplarisch den Rand einer Wachstumssäule, die unter verschiedenen Blickrichtungen unterschiedlichen Kontrast zeigt. 
Für die Aufnahmen in Abbildung 6.18 wurde die Probe schrittweise um die Substratnormale um insgesamt ca. $\pm 7^{\circ}$ verkippt. Der Betrachter soll sein Augenmerk mehr dem oberen Bereich des Films widmen. Man erkennt, dass Säulen, die bei einer Verkippung von z.B. $+3^{\circ}$ einen dunklen Kontrast, bei $-4,5^{\circ}$ einen hellen Kontrast zeigen. Ab einem Verkippungswinkel von ca. $\pm 7^{\circ}$ verschwindet der Kontrast gänzlich. Der maximale in-plane-Orientierungsunterschied der Körner an der Filmoberseite beträgt also $14^{\circ}$, wobei benachbarte Körner einen Orientierungsunterschied von ca. $7^{\circ}$ aufweisen. Zum Vergleich geben die Auswertungen röntgenographischer Messungen einen Wert an, der die Halbwertsbreite der Verteilung über die gesamte Filmdicke angibt.

Scheidet man YBCO auf YSZ-Filmen ab, so keimt der Supraleiter auf nur wenigen Prozent des YSZ-Films an und überwächst viele benachbarten Körner. Das zeigen TEMUntersuchungen an YBCO auf YSZ-Filmen. Die YBCO-Körner sind mit bis zu $500 \mathrm{~nm}$ Durchmesser um ein vielfaches größer als die YSZ-Körner. Der maximale in-planeOrientierungsunterschied von $14^{\circ}$ bei einem $1 \mu \mathrm{m}$ dicke IBAD-YSZ-Film stellt damit den maximalen, zwischen benachbarten YBCO-Körnern auftretenden Korngrenzwinkel dar. Solche Korngrenzen limitieren die kritische Stromdichte im Hochtemperatursupraleiter.

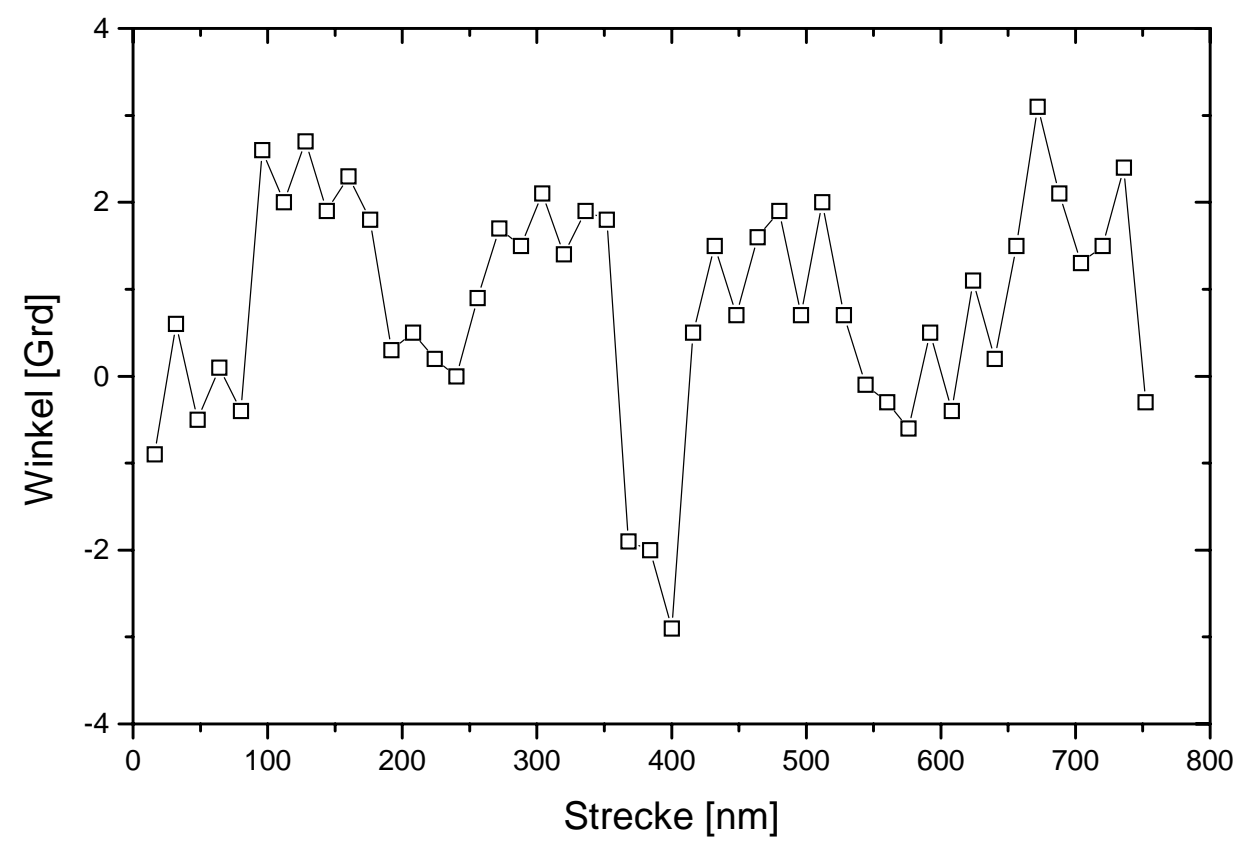

Abbildung 6.19: Relative Änderung der (001)-Orientierung entlang der IBAD-YSZFilmoberseite. Die Messpunkte wurden aus den Positionen der (002)-Reflexe im Beugungsbild bestimmt. 
Die lateralen Orientierungsunterschiede bezüglich der (001)-out-of-plane-Ausrichtung der Säulen zeigt Abbildung 6.19. Hierzu wurde der Elektronenstrahl fokussiert (Durchmesser $<50 \mathrm{~nm}$ ) und die Probe im Mikroskop so bewegt, dass der Elektronenstrahl entlang der Filmoberseite einer im Querschnitt präparierten Probe lief. Die Änderung des Beugungsbildes wurde mit einer Digitalkamera aufgenommen und ausgewertet. Der Nullpunkt der Ordinate wurde in der Auftragung willkürlich gesetzt. In dem gemessenen Bereich von ca. $800 \mathrm{~nm}$ ist zu erkennen, dass benachbarte Körner bis zu maximal $4^{\circ}$ Orientierungsunterschiede aufweisen können, während, wie auf den vorhergehenden Seiten gezeigt, die mittlere Abweichung von der Substratnormalen ca. $1^{\circ}$ beträgt. Die so gemessene laterale Ausdehnung gleichorientierter Bereiche liegt mit durchschnittlich $100 \mathrm{~nm}$ im Bereich der in Abschnitt 6.4 angegeben Korndurchmesser an der Filmoberseite. 


\section{Mechanismen der Texturausbildung}

Die Parameter des unterstützenden Ionenstrahls sind für die konventionelle Herstellung von YSZ-Pufferschichten von entscheidender Bedeutung. So können der Einfallswinkel, die Teilchenart und -energie und die Stromdichte des unterstützenden Ionenstrahls vorgegeben werden. Bei einem Einfallswinkel von $55^{\circ}$ und dem Einsatz von Argon-Ionen mit $300 \mathrm{eV}$ wurde der Einfluss der Stromdichte, d.h. der Intensität des einfallenden Ionenstrahls untersucht. Der Ionenstrahl fördert auf der einen Seite die biaxiale Texturierung, auf der anderen Seite jedoch erhöht sich mit zunehmender Intensität des unterstützenden Ionenstrahls auch der Abtrag der schon deponierten YSZ-Schicht, so dass die Herstellung verlangsamt wird.

Der Einfluss des Ionenbeschusses auf das Filmwachstum in den verschiedenen Wachstumsphasen wird entsprechend der Gliederung im experimentellen Teil dieser Arbeit diskutiert. Insbesondere soll dabei geklärt werden, warum der Ionenbeschuss $\mathrm{zu}$ einer biaxialen Textur führt. Dies wird zu einem Modell des Wachstums von YSZ-Filmen unter Ionenbeschuss führen, welches abschließend zusammenfassend vorgestellt wird.

\subsection{Substratoberfläche und Keimbildung der YSZ-Filme}

Die Entstehung von Wellenstrukturen auf Substratoberflächen unter Ionenbeschuss ist seit Anfang der 60'er Jahre bekannt [Navez et al., 1962]. Hierbei werden unterschiedliche Ausprägungen der Wellenstrukturen beobachtet. Abhängig vom Einschusswinkel und der Energie des Ionenstrahls richten sich z.B. die Wellenvektoren parallel oder senkrecht zur Einschussrichtung aus. Die komplexen Mechanismen, die zur Entstehung solcher Strukturen an der Oberfläche führen, sollen hier nicht diskutiert werden. Weitergehende Analysen zur Entstehung von Wellenstrukturen bei amorphen Festkörpern finden sich bei Bradley und Harper [Bradley und Harper, 1988].

Aufgrund der reinigungsbedingten Oberflächenveränderung tritt eine mesoskopische Strukturierung der Substratoberfläche auf. Die entstandene Wellenstruktur stellt somit die effektive Oberfläche dar, auf der die Keimbildung der IBAD-YSZ-Filme stattfindet. Um den Einfluss der Strukturen der Substratoberfläche auf die Eigenschaften der Filme zu bewerten, können die Resultate zu dünnen Schichten auf massiven, vorgeätzten Substraten und auf Kohlenstoff beschichteten Kupfernetzchen herangezogen werden. Die Kupfernetzchen werden dabei nicht vor der Beschichtung mit dem unterstützenden Ionenstrahl gereinigt, da dieses den Kohlen- 
stofffilm zerstören würde. Ohne unterstützenden Ionenstrahl wachsen die YSZ-Filme auf beiden Substrattypen zunächst amorph auf, wobei es kristalline Anteile gibt, die in beiden Fällen keine nachweisbare Vorzugsorientierungen aufweisen. Ebenso treten keine signifikanten Unterschiede in den kristallinen Anteilen auf.

Bei unter Ionenstrahlunterstützung abgeschiedenen YSZ-Filmen wurde allerdings eine Tendenz zur Keimbildung an den dem unterstützenden Ionenstrahl abgewandten Seiten der Oberflächenwelle (flache Flanke) beobachtet (vgl. Abbildung 5.8 und 5.9). Diese Kristallite sind parallel zu den Ripples größer ausgedehnt als senkrecht dazu (Abbildung 5.10). Möglicherweise fördert der flachere Einfallswinkel des Ionenstrahls an diesen Stellen die Keimbildung aufgrund erhöhter Beweglichkeit der Adatome. Ferner verringert sich die Abtragsrate unter Ionenbeschuss bei Einfallswinkeln, die größer als $60^{\circ}$ sind stark [Nastasi et al., 1996]. Da der kristalline Anteil in ca. $30 \mathrm{~nm}$ dicken YSZ-Filmen jedoch substratunabhängig ist, lässt sich folgern, dass sich im Mittel die Keimbildungsrate und die Keimorientierung durch die Oberflächenstruktur (u.a. Ripple-Periodizität) nicht ändert.

\subsection{Einfluss des unterstützenden Ionenstrahls auf die Keimbildung in YSZ-Filmen im Frühstadium des Wachstums}

In Abschnitt 5.3.3 wurde gezeigt, dass YSZ-Filme, werden sie ohne zusätzlichen Ionenbeschuss abgeschieden, bis zu einer Dicke von ca. $50 \mathrm{~nm}$ amorph aufwachsen (vgl. Abbildung 5.11). Der amorpher Anteil nimmt dabei mit zunehmendem $\eta$-Verhältnis ab. Abbildung 7.1 (geschlossene Punkte) zeigt eine Auftragung der Dicke der amorphen Anfangsschicht bzw. des kristallinen Anteils in Abhängigkeit von der Intensität des unterstützenden Ionenstrahls.

Die lateralen Korndurchmesser der Kristallite in ca. $30 \mathrm{~nm}$ dicken IBAD-YSZ-Filmen vergrößern sich mit zunehmender Beschussintensität des unterstützenden Ionenstrahls leicht. Der durchschnittliche Korndurchmesser ist bei ohne unterstützenden Ionenstrahl hergestellten YSZ-Filmen ca. $20 \mathrm{~nm}$, dagegen beträgt er bei $\eta=2$ ca. $30 \mathrm{~nm}$. Die Anzahldichte der Körner erhöht sich um $6 \times 10^{10} \mathrm{~cm}^{-2}$ auf $10 \times 10^{10} \mathrm{~cm}^{-2}$ (vgl. Tabelle 5.3, Seite 33). Mit steigender Intensität des unterstützenden Ionenstrahls wird also die Dichte der Keimbildungszentren erhöht.

In einer Filmdicke von $10-100 \mathrm{~nm}$ ist die Keimbildungsrate in den mit Ionenstrahlunterstützung abgeschiedenen YSZ-Filmen so hoch, dass es häufig zu einem neuen Ankeimen an schon vorhandene Körner kommt. Dabei wurde zwischen aufeinanderliegenden Körnern keine Epitaxierelation gefunden. Schematisch ist dieses nochmals in Abbildung 7.2 dargestellt. 


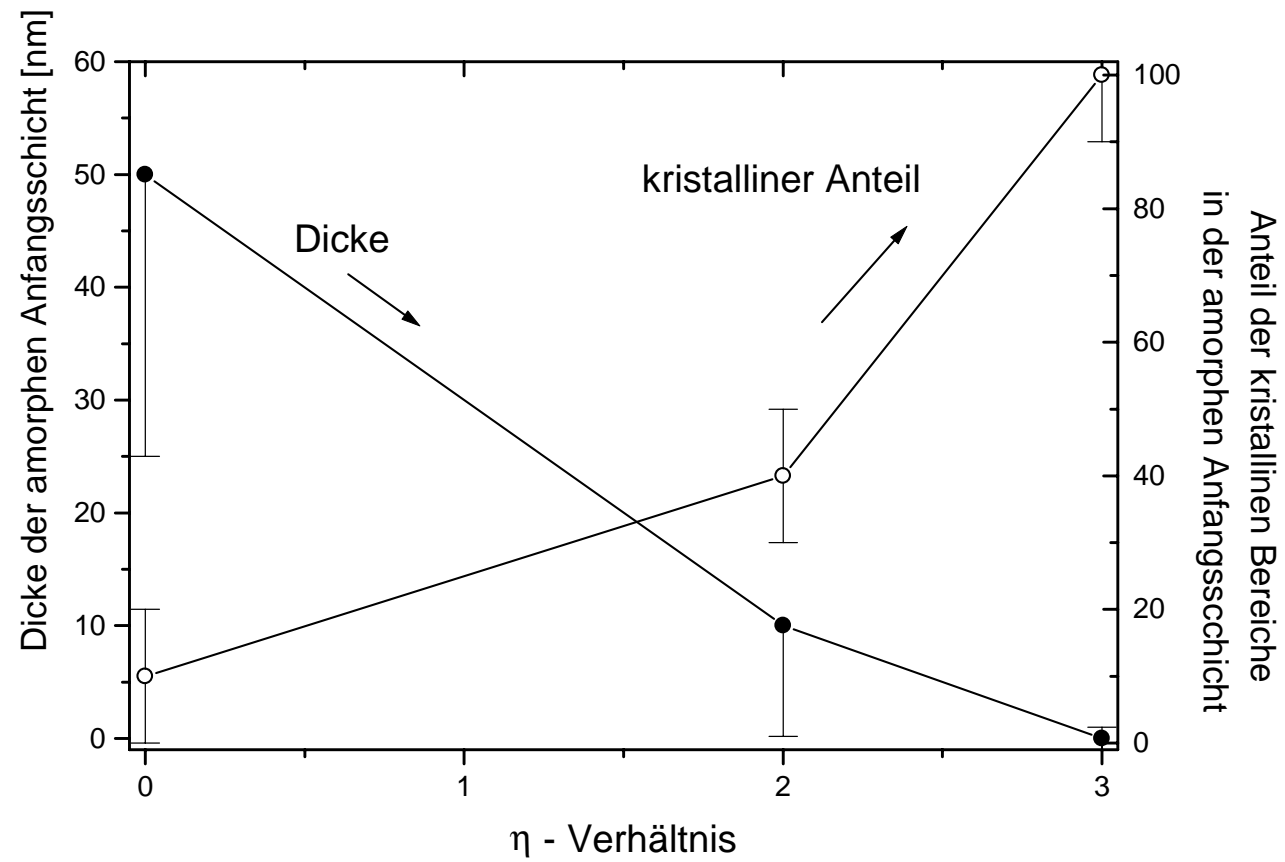

Abbildung 7.1: Dicke der amorphen Anfangsschicht (geschlossene Punkte) und Anteil der kristallinen Bereiche in der amorphen Anfangsschicht (offene Punkte) der bei verschiedenen $\eta$-Verhältnissen abgeschiedenen YSZ-Filme. Die Variation der amorphen Schichtdicke ist als Fehlerbalken eingezeichnet. Die Geraden veranschaulichen den Verlauf.

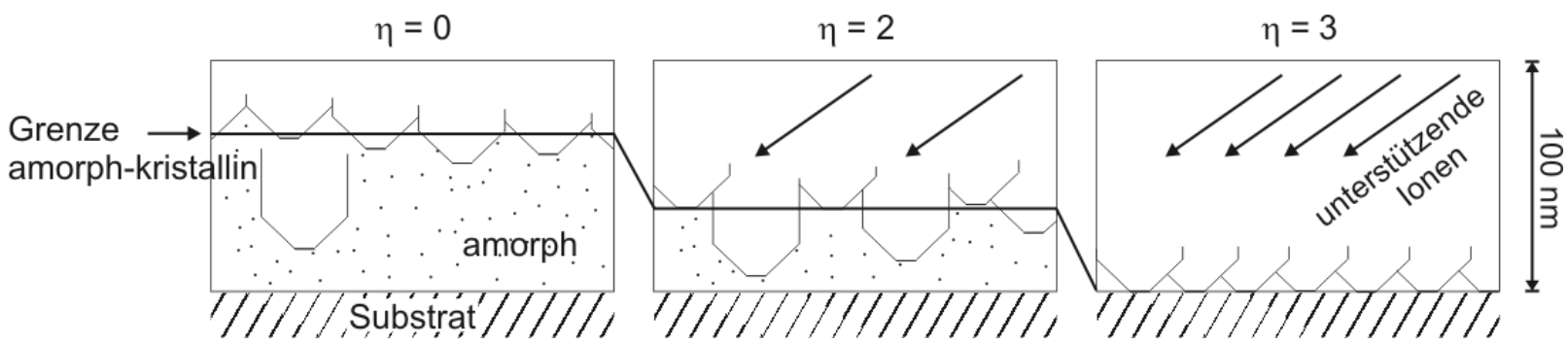

Abbildung 7.2: YSZ-Film im Querschnitt, hergestellt bei verschiedenen $\eta$-Verhältnissen, schematische Darstellung.

Wie in Abschnitt 5.3.3 gezeigt wurde, können in dünnen amorphen YSZ-Filmen auch nach Abschluss der Deposition durch nachträglichen Ionenbeschuss unter typischen IBADParametern kristalline Bereiche erzeugt werden (vgl. Abbildung 5.13). Der durchschnittliche Durchmesser der Kristallite nimmt dabei mit zunehmender Dauer des Ionenbeschusses zu. Da die Untersuchungen nur in Aufsicht an ca. $30 \mathrm{~nm}$ dicken YSZ-Filmen durchgeführt wurden, lassen sich keine Aussagen darüber machen, ob die Kristallite sich nur in den oberflächenna- 
hen Bereichen verbreitern. Beugungsexperimente zeigen, dass die Kristallite keine Vorzugsorientierungen aufweisen.

Die Eindringtiefe der Argon-Ionen des unterstützenden Ionenstrahls mit einer Energie von $300 \mathrm{eV}$ in amorphes $\mathrm{ZrO}_{2}$ wurde mittels des Simulationsprogramm SRIM-2000 (ㅁtopping and

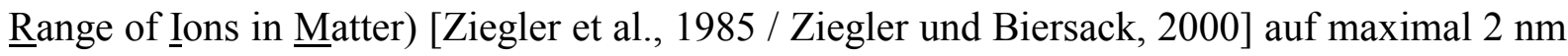
bestimmt. Aufgrund der geringen Eindringtiefe der Argon-Ionen ist es wahrscheinlich, dass nur in oberflächennahen Bereichen eine Kristallisation stattfindet. Die Querschnitts-TEMUntersuchungen haben außerdem gezeigt, dass während des ionenstrahlunterstützten Filmwachstums oberhalb einer einige Nanometer dicken amorphen Schicht ein kristalliner Bereich anwachsen kann, ohne dass eine Rekristallisation des darunter liegenden amorphen Materials auftritt.

Reade und Mitarbeiter verwenden das Verfahren der nachträglichen IonenstrahlTexturierung (ITEX) um dünne amorphe YSZ-Filme zu texturieren. Dabei ist ITEX ein Oberflächen-Texturierungsprozess, der sich nicht auf ein konkurrierenden Kornwachstumsmechanismus stützt. Reade und Mitarbeiter benutzen die typischen Parameter des unterstützenden Ionenstrahls (300 eV Ar, 55 Einfallswinkel), bei einer Beschussdauer von 1,5 min [Reade et al., 2002]. Möglicherweise tritt bei längerem Ionenbeschuss auch ein erneutes Amorphisieren und Rekristallisieren ein, so dass sich letztendlich eine Vorzugsorientierung ausbildet. Die in dieser Arbeit untersuchten Proben wiesen keine Textur auf, so dass es notwendig zu sein scheint, z.B. bei erhöhten Substrattemperaturen zu bestrahlen.

Der unterstützende Ionenstrahl hat also einen entscheidenden Einfluss auf die Keimbildung in der ersten Phase des Filmwachstums. Wie stark der Einfluss ist, verdeutlichen nochmals folgende Zahlen. Bei den verwendeten Depositionsbedingungen wurde jeweils die Energie der Argon-Ionen konstant beibehalten $\left(E_{\mathrm{Ar}}=300 \mathrm{eV}\right)$, lediglich die Stromdichte wurde variiert. Bei einer Stromdichte von $300 \mu \mathrm{A} / \mathrm{cm}^{2}$ treffen ca. $1,9 \times 10^{15}$ Ionen $/ \mathrm{s} \cdot \mathrm{cm}^{2}$ am Substrat auf. Bei einem $\eta$-Verhältnis von $1-2$ werden somit durchschnittlich $300-600 \mathrm{eV}$ pro deponiertem Atom übertragen. Die Eindringtiefe der Argon-Ionen des unterstützenden Ionenstrahls mit einer Energie von $300 \mathrm{eV}$ beträgt maximal $2 \mathrm{~nm}$. Die auftreffenden Argon-Ionen können daher nur im Bereich von einigen Monolagen unter der Filmoberfläche eine direkte Wechselwirkung mit dem deponierten Material erzielen [Miteva et al., 1998 / Ensinger, 1995]. In diesem Bereich wird im Mittel pro Stoß eines Argon-Ions eine Energie auf ein Filmatom übertragen, die größer als die Bindungsenergie von ca. 20 - $30 \mathrm{eV}$ ist [Wiesmann, 1998]. Bei diesen Energien werden ca. $15 \%$ der bereits deponierten Atome wieder abgetragen. 
Aus den in Tabelle 7.1 angegebenen Daten zur Ätzrate und Stromdichte lässt sich die sogenannte Ausbeute $Y$ (Yield) bestimmen:

$$
Y=\frac{\text { Anzahl der ausgelösten Struktureinheiten } \mathrm{ZrO}_{2}}{\text { Anzahl der einfallenden Ionen }}
$$

Typischerweise liegt der Wert zwischen 0,1 und 20, wobei der Sputteryield u.a. von der Masse und der Energie des Ions und vom Targetmaterial abhängt. Bei der hier verwendeten Ionenenergie von $300 \mathrm{eV}$ lösen z.B. ca. 10 Argon-Ionen eine Struktureinheit $\mathrm{ZrO}_{2}$ aus.

\begin{tabular}{|c|c|c|c|}
\hline $\begin{array}{c}\text { Stromdichte } J \text { des unterstützenden } \\
\text { Ionenstahls (Einfallswinkel } 55^{\circ} \text { ) }\end{array}$ & $\eta$-Verhältnis & Ätzrate $[\AA / \mathrm{s}]$ & $\begin{array}{c}\text { Sputteryield } \\
Y\end{array}$ \\
\hline $300 \mu \mathrm{A} / \mathrm{cm}^{2}$ & 2 & 0,5 & 0,08 \\
\hline $450 \mu \mathrm{A} / \mathrm{cm}^{2}$ & 3 & 0,67 & 0,07 \\
\hline
\end{tabular}

Tabelle 7.1: Sputteryield (Sputterausbeute) bei unterschiedlichen IBAD-Bedingungen.

Betrachtet man zunächst das ITEX-Experiment, so zeigt der Vergleich mit IBAD, dass in beiden Fällen die Ionenrate $\left(\frac{\mathrm{d} n}{\mathrm{~d} t}=\frac{J}{e}\right)$ gleich ist. Dagegen ist die Ionendosis $\left(n=\frac{J}{e} \cdot t\right)$ beim IBAD jedoch viel kleiner, da nach ca. 8 Sekunden Deposition $2 \mathrm{~nm}$ Film aufgewachsen sind, d.h., dass nach dieser Zeit der Bereich unterhalb der $2 \mathrm{~nm}$ (max. Eindringtiefe der ArgonIonen) unbeeinflusst bleibt. In Abschnitt 5.3.3 wurde gezeigt, dass durch den nachträglichen Ionenbeschuss keine neuen Keime gebildet werden. Die deponierte Energie führt im wesentlichen zu einem Wachstum der in der amorphen Schicht vorhandenen Kristallite. Da die Kristallite regellos orientiert sind, kann sich somit keine Textur ausbilden. Um einen Film mittels ITEX zu texturieren müssen also Parameter gefunden werden, die zu einer neuen Keimbildung führen, welche Vorrausetzung für die Texturierung ist. Ferner muss der kristalline Anteil im Ausgangsfilm reduziert werden, da diese Bereiche eine statistische Orientierungsverteilung aufweisen.

Die Bildung einer amorphe Anfangsschicht bei YSZ-Filmen, die bei Raumtemperatur abgeschieden wurden, ist zunächst nicht ungewöhnlich, da neben der eingeschränkten Kinetik (Abschreckung) auch die Oberflächenenergie diese Phase begünstigt [Molodetsky et al., 2000]. Die Keimbildung erfolgt überwiegend nicht heterogen, da nur wenige Kristallite an der Substratoberfläche entstehen. Nach dem Zusammenwachsen dieser Kristallite (Koales- 
zenz) bildet sich im weiteren Wachstum keine amorphe Phase mehr. Die Keimbildungsbarriere in der Frühphase scheint daher sehr hoch zu sein, wogegen die Mobilität (Kinetik) der Adatome ausreicht, um relativ schnell große Kristallite $(20-30 \mathrm{~nm})$ auszubilden. Der wesentliche Unterschied zum ITEX-Verfahren ist also die Keimbildung während der ionenstrahlunterstützten Deposition.

In der Literatur werden für die ionenstrahlunterstützte Keimbildung in einer amorphen Matrix verschiedene Mechanismen angegeben. Die auftreffenden Ionen des unterstützenden Ionenstrahls können zum einen die metastabile amorphe YSZ-Schicht abtragen und so eine Oberflächenmorphologie bilden, so dass an Rändern mögliche Keimbildungszentren entstehen können. Durch Stöße der Ionen mit den Atomen des Films werden zum anderen Keimzentren gebildet, die ein kristallines Wachstum bei Raumtemperatur ermöglichen. Die Trajektorie eines Ions im Festkörper endet in einem sogenannten Hot Spot, der durch sehr hohe Temperaturen und Drücke auszeichnet ist. In solchen Bereichen kann das Material bevorzugt kristallisieren [Mizutani, 1995]. Eingebrachte Punktdefekte erhöhen in unterschiedlichem Maß sowohl die Freie Energie der amorphen als auch der kristallinen Phase. Ab einer kritischen Defektdichte haben beide Phasen die gleiche Freie Energie und es kann zu einer Kristallisation kommen [Spinella et al., 1991].

Die Unterschiede zwischen den Texturierungsverfahren liegen also in der erhöhten Keimbildungsrate und der geringeren Ionendosis beim IBAD-Verfahren. Es bleibt unklar, ob die hohe Ionendosis beim ITEX-Verfahren entstandene Kristallite wieder zerstört. In diesem Fall müsste man die Substrattemperatur erhöhen, um ein schnelles Kristallwachstum zu erreichen. So könnten einzelne Keime eine Größe erreichen, die durch einen Ionenbeschuss nicht zerstört werden. Es ist insbesondere denkbar, dass gerade solche Kristallite stabil sind, die so orientiert sind, dass eine große Eindringtiefe für die Ionen vorliegt (vgl. Channeling, nächster Abschnitt), so dass es zu einer Ausbildung einer Vorzugsorientierung kommt.

Möglicherweise existieren beim IBAD-Verfahren jedoch gänzlich andere Verhältnisse als beim nachträgliche Beschuss mit Ionen (ITEX). So spielt beim Wachstum des IBAD-Films u.a. die Kondensation eine wichtige Rolle, wogegen der ITEX-Film nur einer Bestrahlung ausgesetzt wird. Vergleich man z.B. die Korngrößen von IBAD- und ITEX-Filmen (vgl. Abschnitt 5.3.3, Seite 31), so findet man ähnliche Werte trotz sehr unterschiedlicher Ionendosen. Eine Übertragung von ITEX-Ergebnissen auf das IBAD-Verfahren ist sicherlich nur eingeschränkt möglich.

\subsection{Anfangsstadien der Texturentstehung}

Im vorangegangenen Abschnitt wurde das kristalline Anfangsstadium des YSZFilmwachstums durch im wesentlichen (110)- und (001)-orientierte Körner, die aus einer 
amorphen Anfangsschicht entstehen, charakterisiert. Die Vorauswahl der Orientierungen ist, wie noch gezeigt wird, spannungsinduziert. Zunächst soll aber ein Wachstumsmodell von TiN-Filmen zum Vergleich mit YSZ-Filmen herangezogen werden

TiN-Filme, von Li und Mitarbeiter durch Magnetron-Abscheidung hergestellt, zeigen ebenfalls das Vorhandensein einer amorphen Anfangsschicht. Li und Mitarbeiter gehen davon aus, dass die amorphe Phase stabiler ist als die kristalline [Li et al., 2002]. Sie entwickelten auf der Basis ihrer Ergebnisse ein Wachstumsmodell, das in Abbildung 7.3 dargestellt ist. Ab einer kritischen Filmdicke entstehen innerhalb der amorphen Anfangsschicht Keime, welche keine Vorzugsorientierung aufweisen. Die Keime wachsen lateral bis zur Koaleszenz (vgl. Abbildung $7.3 \mathrm{~b}$ ).

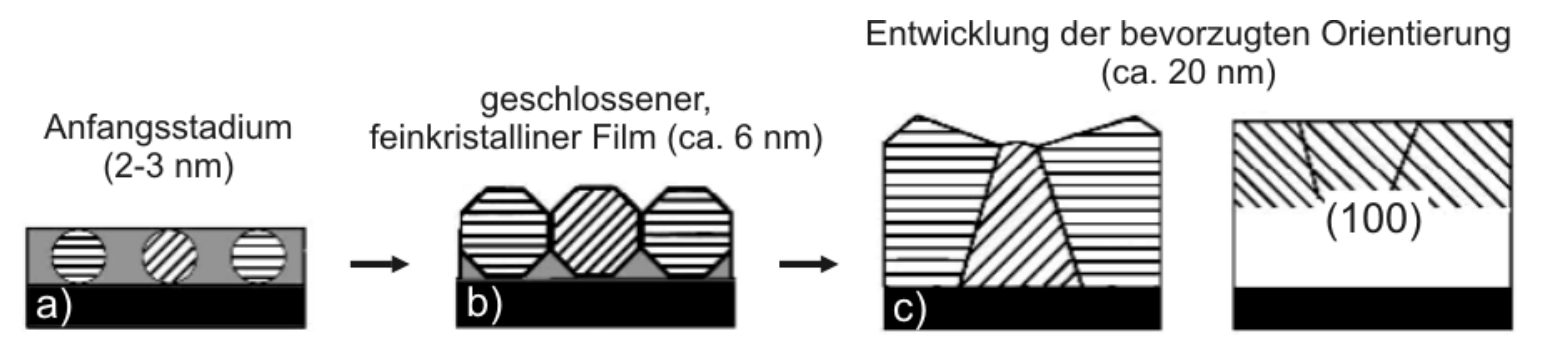

Abbildung 7.3: Wachstumsmodell von TiN-Filmen von Li und Mitarbeitern. a) Nach einer amorphen Anfangsschicht wachsen kristalline Bereiche ohne bevorzugte Orientierungen. $b$ ) Oberhalb einer kritischen Schichtdicke ist der Film kristallin. c) Je nach Depositionsbedingungen ist das bevorzugte Wachstum (111)- oder (100)-orientiert (aus [Li et al., 2002]).

Im weiteren unterscheiden Li und Mitarbeiter zwei Szenarien. Bei eingeschränkter Kinetik tritt ein kolumnares Wachstum auf. Es bildet sich mit zunehmender Filmdicke die Orientierung aus, welche die höchste Wachstumsgeschwindigkeit hat und sich durch ein Überwachsen durchsetzten kann [van der Drift, 1968]. Für TiN ist dies die (111)-Orientierung [Pelleg et al., 1991]. Bei einer ionenstrahlunterstützten Deposition führt aber eine erhöhte Korngrenzdiffusion, Rekristallisationseffekte oder die sekundäre Keimbildung zur Ausbildung einer Vorzugsorientierung, so dass sich die Kristallebenen mit der geringsten Oberflächenenergie ((001) für TiN) parallel zum Substrat bilden [Li et al., 2002]. Li und Mitarbeiter gehen aber davon aus, dass der Einfluss der Ionenbestrahlung in dickeren Schichten kleiner wird, so dass sich in späteren Wachstumsstadien wieder die Orientierung mit der höchsten Wachstumsgeschwindigkeit durchsetzt. Sie erklären so den schichtdickenabhängigen Übergang von einer (001)- zu einer (111)-Textur [Li et al., 2002].

Es ist zweifelhaft, ob sich dieses Modell auf IBAD-YSZ übertragen lässt, da z.B. die Anfangsschicht beim IBAD-YSZ nicht polykristallin ist. Vielmehr enthält sie neben den (110)- 
und (001)- praktisch kaum (111)-Anteile. Es muss also schon bei der Keimbildung in der amorphen Phase ein Selektionsmechanismus existieren. Des weiteren sollte nach den Berechnungen von Christensen und Carter die (110)-Orientierung von kubischem $\mathrm{ZrO}_{2}$ eine geringere Oberflächenenergie aufweisen als die (001)-Orientierung [Christensen und Carter, 1998]. Es ist aber im Sinne des Modells von Li und Mitarbeitern nicht verständlich, warum beim IBAD-YSZ der (110)-Anteil mit zunehmenden $\eta$-Verhältnis (erhöhter Ionenrate) sukzessive abnimmt (vgl. Abschnitt 5.2, Seite 23).

Daneben macht Dzick in seinen Beobachtungen für das Vorhandensein der (110)orientierten Bereiche eine spannungsinduzierte Vorselektion der Orientierung verantwortlich. Danach sollte mit zunehmendem $\eta$-Verhältnis zunächst die Bildung der (111)- und dann der (110)-Orientierung unterdrückt werden. Er geht dabei von einer (110)-Drahttextur mit geringen (001)-Anteilen aus [Dzick, 2000]. TEM-Untersuchungen fanden dagegen keine ausgeprägte (110)-Drahttextur.

111200220

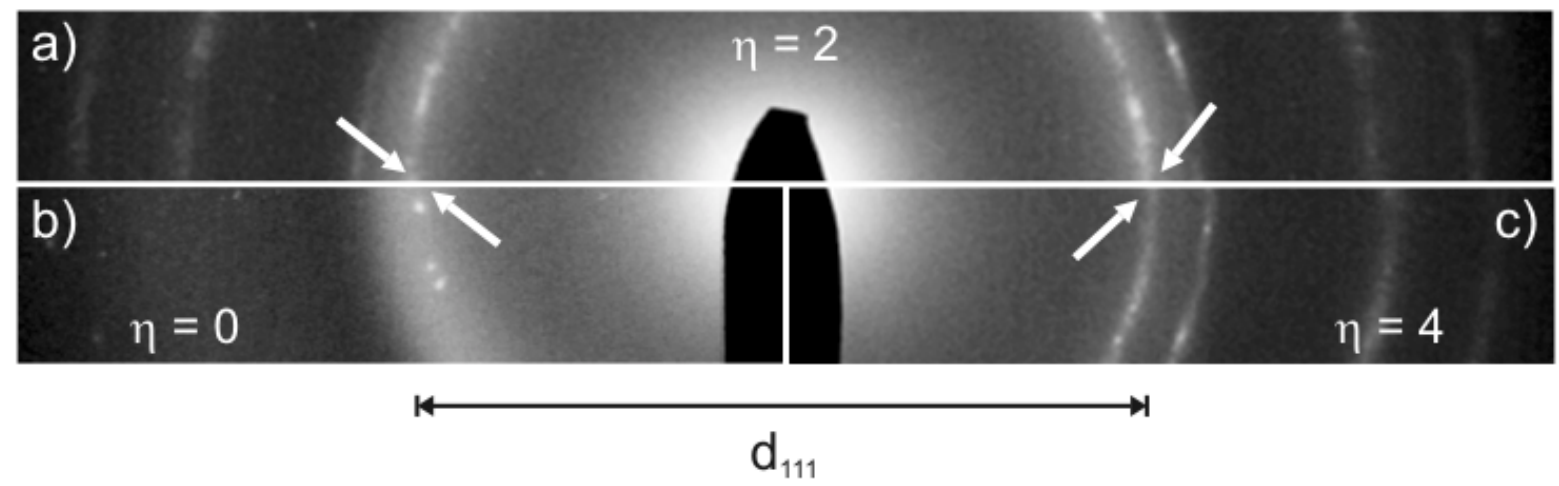

Abbildung 7.4: Feinbereichsbeugungsbilder, Montage. Für dieses Bild wurden die SADBeugungsbilder von Proben, die mit a) $\eta=0, b) \eta=2$ und c) $\eta=4$ abgeschieden wurden, zusammengefügt. Die Pfeile deuten auf die Aufweitung der Ringe mit zunehmender Intensität des unterstützenden Ionenstrahls. Die Aufweitung der (hkl)-Ringe ist gleichbedeutend mit einer Verringerung der jeweiligen ( $h k l)$-Netzebenenabständen.

Vergleicht man die Feinbereichsbeugungsbilder, die an mit YSZ beschichteten Kohlenstoffnetzchen in Aufsicht gemacht wurden, so erkennt man, dass die Radien der Beugungsringe mit steigendem $\eta$-Verhältnis zunehmen, d.h., dass sich die Netzebenenabstände verringern. In Abbildung 7.4 sind die Feinbereichsbeugungsbilder der bei verschiedenen $\eta$-Verhältnissen abgeschieden, ca. 30 - $60 \mathrm{~nm}$ dicken YSZ-Filme in einer Montage zu sehen. Die Pfeile deuten auf die Aufweitung der Beugungsringe. Da die Kameralänge des Mikroskops nicht eindeutig bekannt ist, ist es nicht möglich, aus dem Radius des Beugungsringes ( $h k l)$ einen absoluten 
Netzebenenabstand $d_{h k l}$ zu bestimmen. Mit Hilfe des Durchmesser $d_{h k l}$ lassen sich aber die relativen Dehnungen $\varepsilon_{\mathrm{hkl}}$ bestimmen:

$$
\varepsilon_{h k l}=\frac{d^{1}{ }_{h k l}-d^{2}{ }_{h k l}}{d^{2}{ }_{h k l}}
$$

Dazu wurden die Durchmesser der Beugungsringe des ohne unterstützenden Ionenstrahls abgeschiedenen Films als Referenz angenommen. Diese Werte sind allerdings nicht mit absoluten Dehnungen zu vergleichen, da die ohne unterstützenden Ionenstrahl abgeschiedenen YSZFilme schon Verspannungen aufweisen, das zeigen die verschobenen Reflexlagen in Röntgenmessungen (vgl. Abbildung 5.3, Seite 23). Die ermittelten relativen Dehnungen sind in Tabelle 7.2 aufgeführt. Da sich die mittels TEM bestimmten Werte auf die Dehnung in der Filmebene beziehen, sind zum Vergleich auch die aus Röntgenmessungen bestimmten relativen Dehnungen parallel zur Substratnormalen angegeben. Da auch in YSZ-Filmen, die ohne Ionenstrahlunterstützung hergestellt wurden, Druckspannungen beobachtet werden, stellen die angegebenen relativen Dehnungen eine untere Grenze dar. Es treten also schon in sehr dünnen Filmen beträchtliche Spannungen auf, so dass der von Dzick angegebene Mechanismus zu einer Unterdrückung der (111)-Orientierung in der Frühphase führen kann.

\begin{tabular}{|c|c|c|}
\cline { 2 - 3 } \multicolumn{1}{c|}{} & \multicolumn{2}{c|}{ relative Dehnung $\varepsilon_{\mathrm{hkl}}$, ermittlet aus... } \\
\cline { 2 - 3 } & $\begin{array}{c}(111)- \\
\text { Beugungsringe }\end{array}$ & $\begin{array}{c}(200)- \\
\text { Röntgenpeaks }\end{array}$ \\
\hline$\eta=0$ & $\equiv 0$ & $\equiv 0$ \\
\hline$\eta=2$ & $(-1 \%)-(-2 \%)$ & ca. $2 \%$ \\
\hline$\eta=4$ & $(-3 \%)-(-4 \%)$ & ca. $3 \%$ \\
\hline
\end{tabular}

Tabelle 7.2: Relative Dehnung, bestimmt aus den Beugungsringdurchmesser und Röntgenmessungen, der bei verschiedenen $\eta$-Verhältnissen abgeschiedenen YSZ-Filmen. Die Aufweitung der Ringdurchmesser bzw. Verschiebung der Röntgenreflexe zu kleineren Winkeln ist gleichbedeutend mit einer Verringerung bzw. Aufweitung der jeweiligen Netzebenenabstände.

Sowohl die Kompression in der Filmebene als auch die Dilatation in Wachstumsrichtung weisen somit auf laterale Druckspannungen hin. Ein wichtiger Mechanismus für die bei der Filmdeposition entstehenden Druckspannungen ist das sogenannte atomic peening [D'Heurle, 1970 / D'Heule und Harper, 1989 / Hofmann, 1990 / Thornton et al., 1998 / Windischmann, 1987 / Windischmann, 1992 / Window, 1993]. Bei energiereichem Beschuss vollziehen Film- 
atome durch Kollisionen Platzwechsel. Diese sind durch die Einschussrichtung des Beschussteilchens auch in die Filmtiefe gerichtet, was zu einer Verdichtung des deponierten Materials führt. So können Punktdefekte entstehen, deren Spannungsfelder sich zu hohen Gesamtspannungen summieren.

Ein weiterer Hinweis, der die besondere Bedeutung von Spannungen in der Struktur- und Phasenbildung zeigt, ist das Auftreten der monoklinen Phase des $\mathrm{ZrO}_{2}$, die in Filmdicken bis ca. $100 \mathrm{~nm}$ gefunden wurde. Die monokline Phase lässt sich nur durch den Winkel zwischen den (100)- und (001)-Ebenen $\left(\beta \approx 100^{\circ}\right)$ identifizieren [McCullough und Trueblood, 1959] und wurde daher nur selten nachgewiesen. In Abbildung 7.5 ist die monokline Phase in Hochauflösung dargestellt.

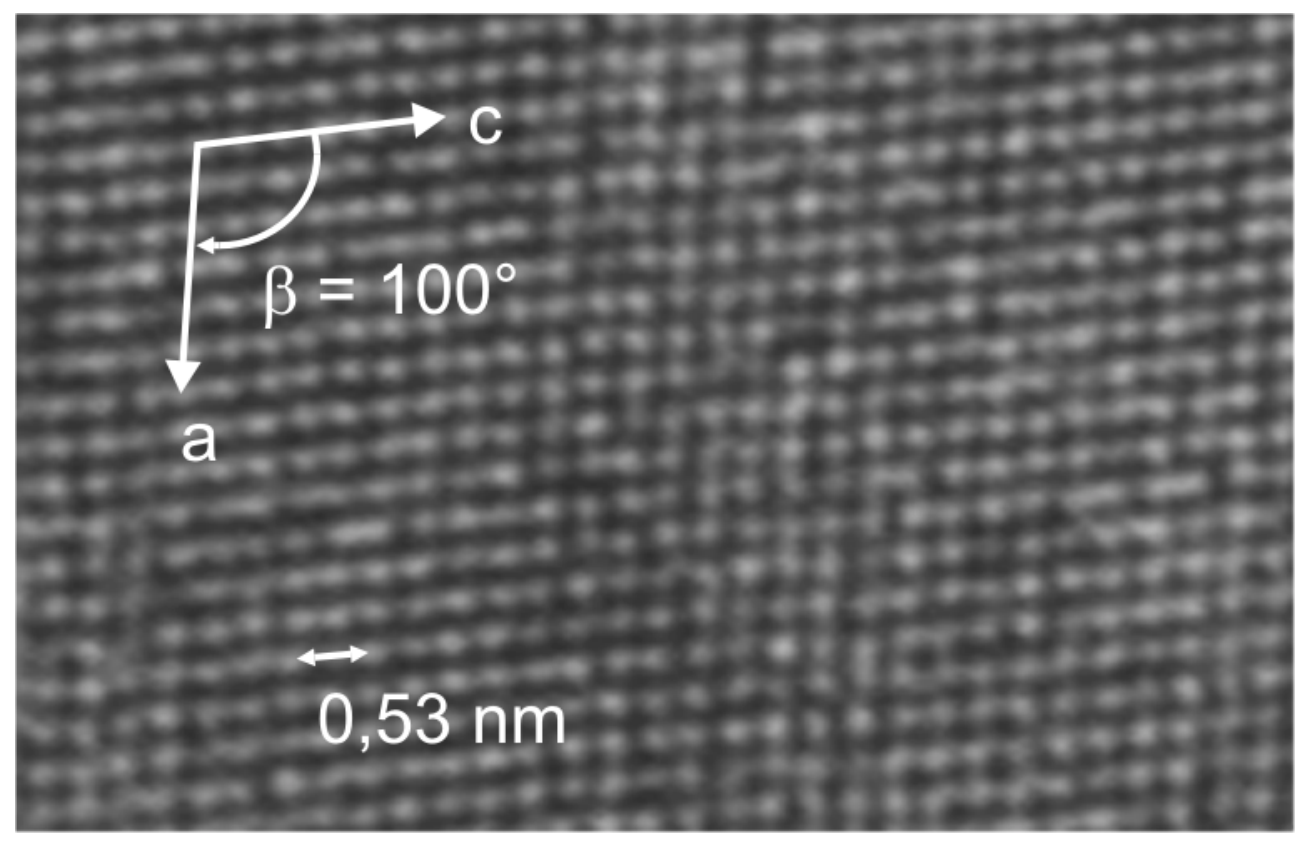

Abbildung 7.5: HREM-Aufnahme der monoklinen $\mathrm{ZrO}_{2}$-Phase, wie sie in Fimdicken von ca. $80-100$ nm gefunden wurde. Diese Phase lässt sich nur in $\langle 010\rangle-$ Blickrichtung nachweisen und wurde daher selten beobachtet.

Akimov und Mitarbeiter beobachteten bei der hydrostatischen Verformung von partiell stabilisierten $\mathrm{ZrO}_{2}$ (PSZ) einen Phasenübergang von der tetragonalen- zur monoklinen Phase. Sie führen den Übergang auf eine über Versetzungen vermittelte Entmischung zurück, bei der Yttrium in den Korngrenzen angereichert wird [Akimov et al., 1994]. Aufgrund der geringen Auflösung der energiedispersiven Röntgenanalyse (EDX) im Elektronenmikroskop konnte eine erhöhte Yttrium-Konzentration in den Korngrenzen nicht nachgewiesen werden. Es scheint aber durchaus möglich, dass in dieser Wachstumsphase aufgrund hoher Spannungen Entmischungsprozesse auftreten, die die monokline $\mathrm{ZrO}_{2}$-Phase bilden. 


\subsection{Wachstumsselektion und biaxiale Texturierung}

Im vorigen Abschnitt wurden die Filmspannungen als wesentliche Ursache der Unterdrückung der (111)-Orientierung in der Frühphase und der Ausbildung der (001)Vorzugsorientierung im späteren Wachstumsstadium angesehen. Es müssen aber auch die Mechanismen berücksichtigt werden, die zur Entstehung einer in-plane-Textur führen. Im Vergleich zur out-of-plane-Textur führen sie in einem späteren Stadium des Filmwachstums zu einer ausgeprägten in-plane-Textur (vgl. Abschnitt 5.4). So gibt Dzick für die Entstehung der (001)-Textur an, dass (001)-orientierte Körner bevorzugt auf (110)-orientierten Körnern epitaktisch aufwachsen, wenn eine Ausrichtung der $<111>$-Richtung parallel zum einfallenden Ionenstrahl gewährleistet ist [Dzick, 2000]. In seinem Modell kommt es somit zu einer unstetigen Entstehung der in-plane-Textur.

Mittels Beugungsexperimenten im Querschnitt kann ein solcher Textur-Offset nicht nachgewiesen werden, da über ein zu großer Filmdickenbereich (ca. $100 \mathrm{~nm}$ ) gemittelt wird. Es konnten allerdings in hochaufgelösten Querschnittsbildern keine Epitaxie-Konfiguration gefunden werden, die ein Wachstum von (001)- auf (110)-Körnern bestätigt. In dieser frühen Wachstumsphase dominiert vielmehr der Prozess des Überwachsens, wie sie z.B. in Abbildung 6.9 gezeigt wurde.

Des weiteren sollte sich, nach dem Modell von Dzick, die Textur in dünnen Schichten verschlechtern, wenn sie bei noch höheren $\eta$-Verhältnissen $(\eta>2)$ abgeschieden werden, da mit zunehmender Intensität des Ionenstrahls der (110)-Anteil in der Frühphase des Filmwachstums reduziert wird (vgl. Abbildung 7.1). So erklärt Dzick die Texturunterschiede zu den von Iijima und Mitarbeitern bei höherem $\eta$-Verhältnis abgeschiedenen YSZ-Filmen $(\eta=3,4 \mathrm{im}$ Vergleich zu $\eta=2$ ). Diese zeigen einen schlechteren Textur-Offset (vgl. Abbildung 5.1) [Dzick, 2000 / Iijima et al., 1998]. Bei den für diese Arbeit untersuchten Proben zeigte sich aber, dass für ca. $300 \mathrm{~nm}$ dicke Filme die out-of-plane-Textur bei kleinem $\eta$-Verhältnis (höherer (110)-Anteil) schlechter ist als bei höheren $\eta$-Verhältnissen (vgl. Tabelle 5.5, Seite 47).

Die Ausbildung der (001)-Textur in IBAD-YSZ-Filmen, die bei $\eta=2$ abgeschieden wurden, geschieht bis zu einer Filmdicke von $200 \mathrm{~nm}$, wobei ein Großteil des Films schon nach $50 \mathrm{~nm}$ Filmdicke eine (001)-Orientierung aufweist (vgl. Abbildung 5.22). Ohne unterstützenden Ionenstrahl erhält man einen Film, der zwar größtenteils (001)-Säulen besitzt aber keine in-plane-Textur aufweisen kann. Die von Dzick mittels Röntgenmessungen bestimmten Texturänderungen zeigen, dass die out-of-plane-Textur über die gesamte Filmdicke konstant bleibt (ca. $6^{\circ}$ FWHM), wogegen sich die in-plane-Textur mit zunehmender Filmdicke exponentiell verbessert [Dzick, 2000]. Dagegen zeigen Iijima und Mitarbeiter sowohl für die in- als auch out-of-plane-Textur eine Verbesserung mit zunehmender Filmdicke mit einer Sättigung bei ca. 600 nm Filmdicke (vgl. Abbildung 5.1) [Iijima et al., 1998 / Iijima et al., 1998a]. 
Die Beugungsexperimente an Querschnittsproben zeigten einen exponentiellen Verlauf der out-of-plane-Textur mit einer leichten Sättigung bei $500 \mathrm{~nm}$ Filmdicke. Sowohl diese Ergebnisse als auch Untersuchungen der in-plane-Textur, die ebenfalls auf einen Verlauf mit Sättigung hindeuten, zeigen, dass der wahre Verlauf eher dem von Iijima und Mitarbeitern bestimmten ähnlich ist.

Für die Ausbildung der biaxialen Textur werden in der Literatur im wesentlichen zwei Mechanismen diskutiert:

\section{- Anisotropie der Ätzrate}

MD-Simulationen von Metallfilmen unter Ionenbeschuss (40 eV - $120 \mathrm{eV})$ zeigen, dass für eine Wachstumsselektion ein Höhenvorteil von nur 5 Gitterzellen zwischen zwei benachbarten Körnern nötig ist [Ying et al., 1996 / Dong et al., 1998 / Dong et al., 1999]. Bei Höhenunterschieden von mehr als 20 Gitterzellen können beim Überwachsen Poren entstehen. Solche Poren konnten in den elektronenmikroskopischen Untersuchungen nicht nachgewiesen werden. Die hohe Oberflächenbeweglichkeit der Atome verhindert die Bildung der Poren beim Wachstum.

Ein Höhenvorteil kann dann entstehen, wenn durch eine orientierungsabhängige Sputterrate fehlorientierte Körner stärker abgetragen werden. Dieses wird auf Channelingseffekte zurückgeführt [Behrisch, 1981]. Dringen Ionen entlang einer kristallographischen Richtung in ein Korn ein, so wird die Energie der Ionen in tieferen Schichten des Films deponiert. Bei schlecht orientierten Körnern erfolgt somit ein Abtrag, so dass diese Körner überwachsen werden.

Experimente an YSZ-Einkristallen, die unter verschiedenen Orientierungen abgeätzt wurden bestätigen die anisotropen Ätzraten und zeigen, dass eine deutliche Absenkung der Ätzrate nur beobachtet werden kann, wenn eine $<111>$-Richtung des YSZ zum Ionenstrahl ausgerichtet ist, Ausrichtungen von $<100>$ - oder $<110>$-Richtungen haben deutlich höhere Ätzraten [Dzick, 2000]. Ressler und Mitarbeiter finden allerdings keine signifikanten Unterschiede der Ätzraten der $<110>$ - und <111>-Richtungen [Ressler et al., 1997a].

Für solche Vorstellungen spricht, dass in der mittleren Filmwachstumsphase das Überwachsen der dominierende Prozess ist. In dickeren Filmen wird aber auch weiterhin eine geringe Texturverbesserung beobachtet, ohne das weiteres Überwachsen stattfindet. Zudem zeigen vergleichbare Messungen an anderen Systemen, dass eine ionenstrahlunterstützte Texturierung auch dann möglich ist, wenn die Ätzraten nicht die erwarteten Anisotropien zeigen [Thiele et al., 2002]. 


\section{- Ionenstrahleninduzierte Schädigung}

Wurden für die o.g. Wachstumsselektion Channelingseffekte verantwortlich gemacht, werden beim Modell der ionenstrahlinduzierten Schädigung von Ressler und Mitarbeitern aufgrund der geringen Ionenenergie $(<300 \mathrm{eV})$ Channelingseffekte ausgeschlossen [Ressler et al., 1997a / Ressler et al., 1997b]. In ihrem Modell kommt es durch den Ionenstrahl zu einer Anreicherung von Defekten in oberflächennahen Bereichen, die zur Bildung von Kleinwinkelkorngrenzen führen und somit eine Orientierungsänderung hervorrufen können. Solche Orientierungsänderungen konnten in hochaufgelösten elektronenmikroskopischen Bildern der ersten ca. $200 \mathrm{~nm}$ YSZ-Filmdicke beobachtet werden (siehe Abschnitt 6).

Im folgenden wird die kontinuierliche Orientierungsänderung der in Abbildung 6.2 gezeigten Wachstumssäule genauer diskutiert. Diese Säule ist so orientiert, dass man stets in $<110>$ Richtung des YSZ sieht, das zeigen Fourier-Transformationen an verschiedenen Stellen der Aufnahme. Mit der Änderung der $<001>$-Richtung in der Bildebene erfolgt somit eine Rotation parallel zur Blickrichtung.

Um im Verlauf des Kristallwachstums eine Verkippung der Netzebenen um einen Relativwinkel $\Theta$ zu erreichen, muss ein Überschuss an Stufenversetzungen mit gleichen Burgersvektoren auftreten. Ferner müssen die Linienelemente der Stufenversetzungen parallel zur Rotationsachse verlaufen. Versetzungsverteilungen mit mittlerem Burgersvektor $\langle\underline{\underline{b}}\rangle=0$ tragen nicht zu einer Verkippung bzw. Rotation bei.

Sowohl im HREM-Bild, welches in Abbildung 6.2 gezeigt ist, als auch im fouriertransformiertem Bild (Abbildung 6.7) wurden die Versetzungen ausgezählt. Die Versetzungsdichte in Filmdicken bis ca. $100 \mathrm{~nm}$ beträgt ca. $10^{12} \mathrm{~cm}^{-2}$ und gibt eher einen unteren Grenzwert an, da nur sichtbare Versetzungen, d.h. Versetzungen, deren Linienelement nahezu parallel zur Blickrichtung verlaufen ( $<110>$-Richtung), gezählt wurden. Dieses entspricht einem mittleren Abstand der Versetzungen von ca. $10 \mathrm{~nm}$. Die Versetzungsverteilung ist bis auf eine leichte Anhäufung an Säulengrenzen im wesentlichen homogen. Der Burgersvektor wurde mittels Burgersvektorumläufe bestimmt und beträgt $\underline{b}=a / 2<\overline{1} 11>$. Da das Linienelement (= Blickrichtung) mit $<110>$ senkrecht auf dem Burgersvektor steht, handelt es sich somit um Stufenversetzungen.

Es lässt sich leicht zeigen, dass eine Versetzungsdichte $\rho_{V}$ von Stufenversetzungen, deren Linienelemente in der Filmebene liegen und parallel verlaufende Burgersvektoren $\underline{b}$ haben, zu einer relativen Verdrehung der Netzebenen führen kann. Die Verkippung $\Theta$ lässt sich bei bekanntem $\rho_{V}$ aus dem geometrischen Zusammenhang 


$$
h=\frac{|\underline{b}|}{2 \sin \Theta / 2}
$$

abschätzen, mit $h=\frac{1}{\rho_{V} \cdot d}$, dem mittleren Abstand paralleler Stufenversetzungen mit Burgersvektor $\underline{b}$ und der Korngrenzlänge $d$ (siehe auch [Read und Shockley, 1950]).

Nach Gl. 7.3 beträgt somit die Versetzungsdichte, die notwendig ist eine Verkippung von $30^{\circ}$ innerhalb von ca. $100 \mathrm{~nm}$ Filmdicke zu erzeugen, etwa $10^{12} \mathrm{~cm}^{-2}$. Dieser berechnete Wert ist in Übereinstimmung mit dem ermittelten Wert.

Versetzungen können sich in Ionenkristallen allgemein sehr schwer bewegen, somit ist es unwahrscheinlich, dass sich die Versetzungen z.B. durch Spannungen zu bestimmten Netzwerken anordnen [Friedel, 1964]. Solche Netzwerke konnten auch in den untersuchten IBADYSZ-Filmen nicht beobachtet werden. Insbesondere findet man keine korngrenzähnlichen Anhäufungen. Allerdings deuten die beobachteten Defekthäufigkeiten an Säulengrenzen auf eine mögliche bevorzugte Versetzungsbewegung (zur oder von der Grenze) hin. Bei den gefundenen Versetzungsdichten und deren Abbildung im TEM lässt es sich schwer nachweisen, ob Burgersvektoren einer Richtung dominieren. Detailbilder von YSZ, wie in Abbildung 7.7 dargestellt, zeigen, dass Versetzungen unterschiedlicher Burgersvektoren auftreten können, ohne dass man über deren Häufigkeit signifikante Aussagen treffen kann.

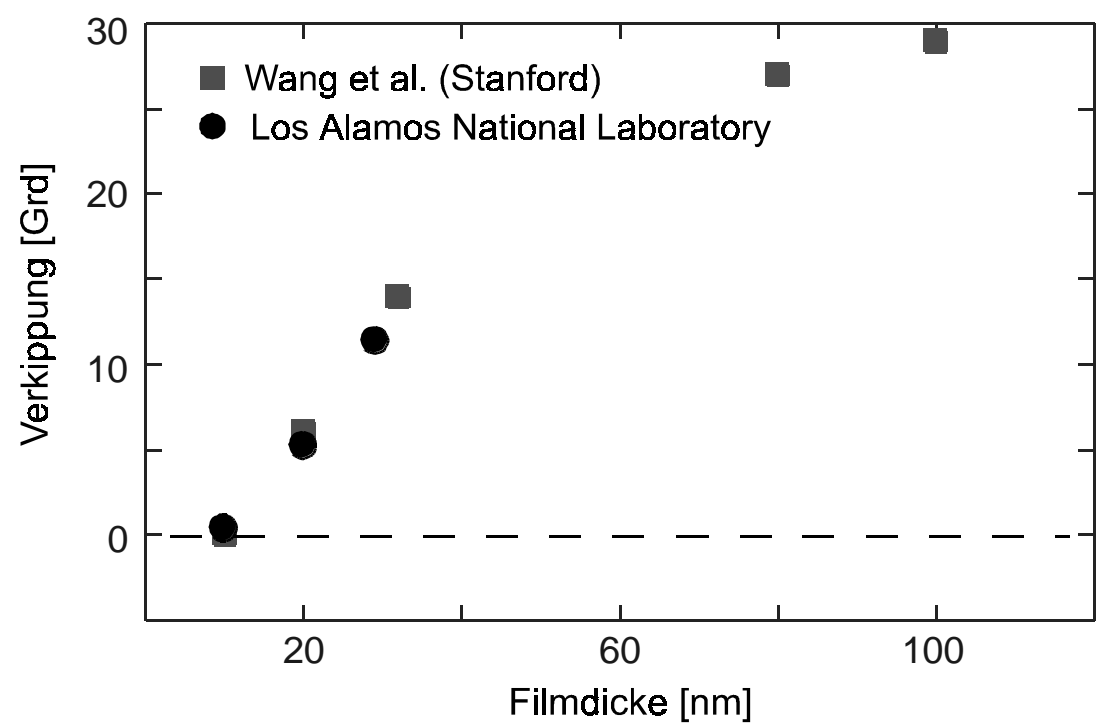

Abbildung 7.6: Verkippung einer Ebenenschar mit zunehmender Filmdicke in IBAD-MgOFilmen (aus [Kung et al., 2001]). 
Eine kontinuierliche Änderung der Orientierung wird auch in anderen Systemen beobachtet. So ist bei mittels Ionenstrahlunterstützung hergestellten MgO-Filmen eine Verkippung bzw. Verdrehung der Orientierung von $30^{\circ}$ innerhalb von $100 \mathrm{~nm}$ Filmwachstum zu erkennen (vgl. Abbildung 7.6). Im Gegensatz $\mathrm{zu}$ der in dieser Arbeit beobachteten out-of-planeOrientierungsänderung geschieht die Änderung im IBAD-MgO-Film jedoch in der Ebene [Wang et al., 1997].

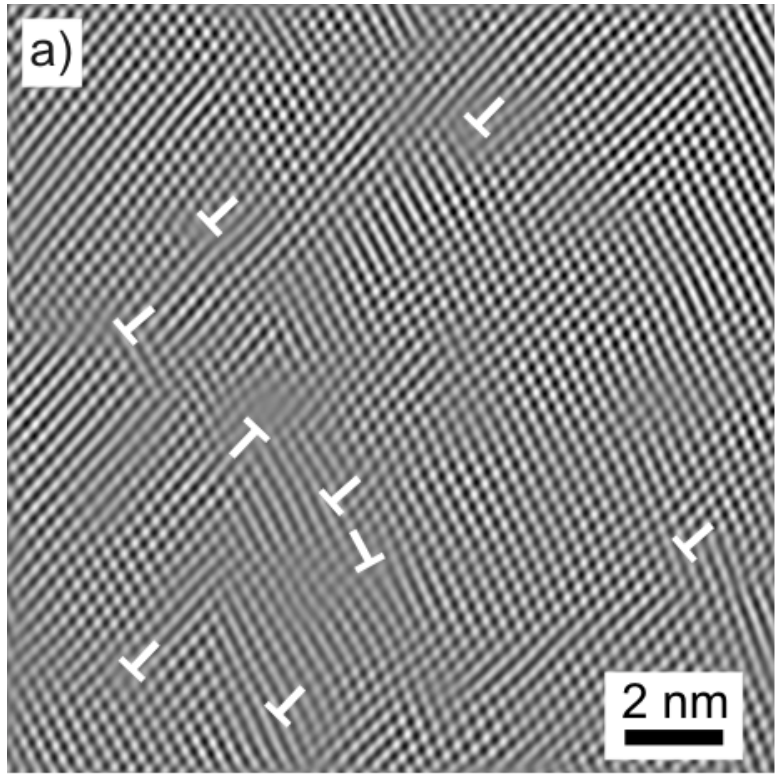

IBAD-YSZ (Querschnitt)

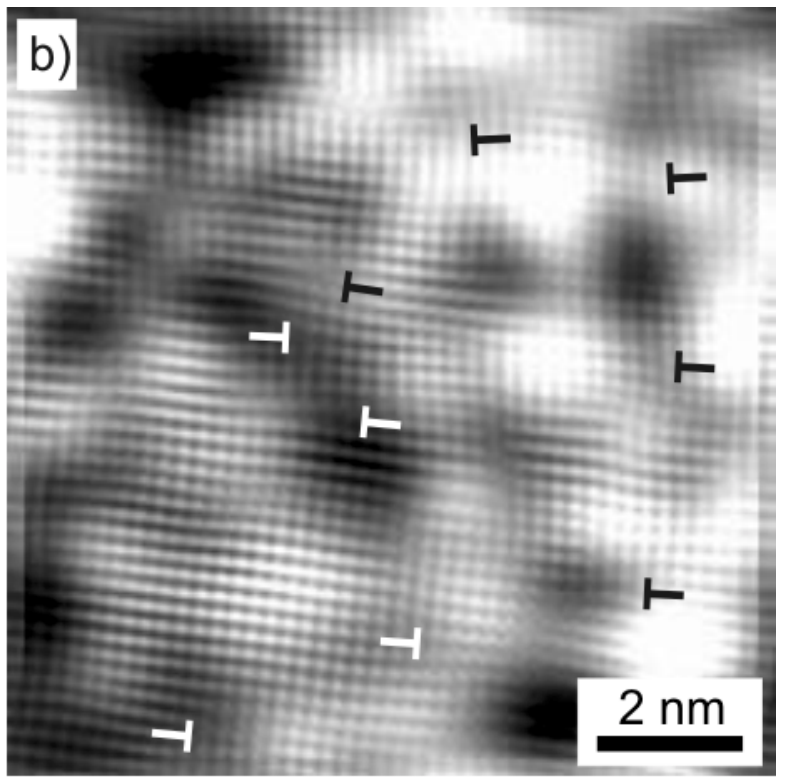

IBAD-MgO (Aufsicht)

Abbildung 7.7: Versetzungen in verschiedenen IBAD-Filmen. Dargestellt sind inverse Fourier-Transformationen von hochaufgelösten TEM-Aufnahmen: a) IBAD-YSZ-Film im Querschnitt, <110>-Blickrichtung, man sieht die $\{111\}_{Y S Z}$-Ebenen, b) IBAD-MgO-Film in

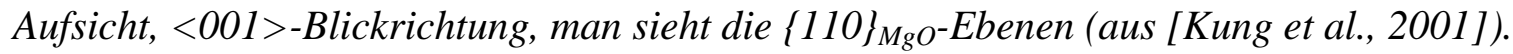

Wie in den in dieser Arbeit untersuchten IBAD-YSZ-Filmen beträgt die Dichte der Versetzungen in den IBAD-MgO-Filmen ca. $10^{12} \mathrm{~cm}^{-2}$. In den MgO-Filmen geht man davon aus, dass sich Punktdefekte so anordnen, dass eingeschobene $\{110\}$-Ebenen entstehen [Kung et al., 2001]. Abbildung 7.7 zeigt zum Vergleich inverse Fourier-Transformationen von IBADYSZ (Querschnitt) und IBAD-MgO (Aufsicht), die die Lage der Versetzungen verdeutlichen.

Die Versetzungsdichte verringert sich in $1 \mu \mathrm{m}$ dicken YSZ-Filmen um einen Faktor 10 auf ca. $10^{11} \mathrm{~cm}^{-2}$ nahe der Filmoberseite. Dadurch, dass in der frühen Wachstumsphase mehr Missorientierungen auftreten kommt es hier wahrscheinlich zu der erhöhten Versetzungsdichte. Die hohen Zahlen deuten aber darauf hin, dass Versetzungen charakteristisch für das Filmwachstum unter den beschriebenen Bedingungen sind. Es stellt sich ein Gleichgewicht 
von Orientierungsverbesserung und Defekteinbau ein. Möglicherweise erklärt dies, warum die Textur nicht beliebig gut wird.

Bei der in dieser Arbeit beschriebenen kontinuierliche Änderung der Orientierung handelt es sich um ein out-of-plane-Orientierungsänderung, die Orientierungsänderung in IBAD-MgO erfolgt in-plane. Ein Vergleich beider Prozesse zeigt aber, dass der Mechanismus der kontinuierlichen Orientierungsänderung zur Einstellung der biaxialen Textur auch durch Kombination beider Prozesse geschehen kann. Es liegt nahe, dass der Mechanismus der kontinuierlichen Orientierungsänderung (in- und out-of-plane) ab einer Filmdicke von ca. $500 \mathrm{~nm}$ der dominierende ist, da die Texturverbesserung hier nur noch langsam vonstatten geht und es auch nicht zu erneuten Ankeimen von Körnern kommt.

\subsection{Gesamtmodell: Texturentstehung in YSZ}

In Abbildung 7.8 ist das Wachstum von YSZ-Filmen, die unter verschiedenen $\eta$ Verhältnissen abgeschieden wurden, schematisch dargestellt. Die Texturentwicklung lässt sich in verschiedene Phasen unterteilen.

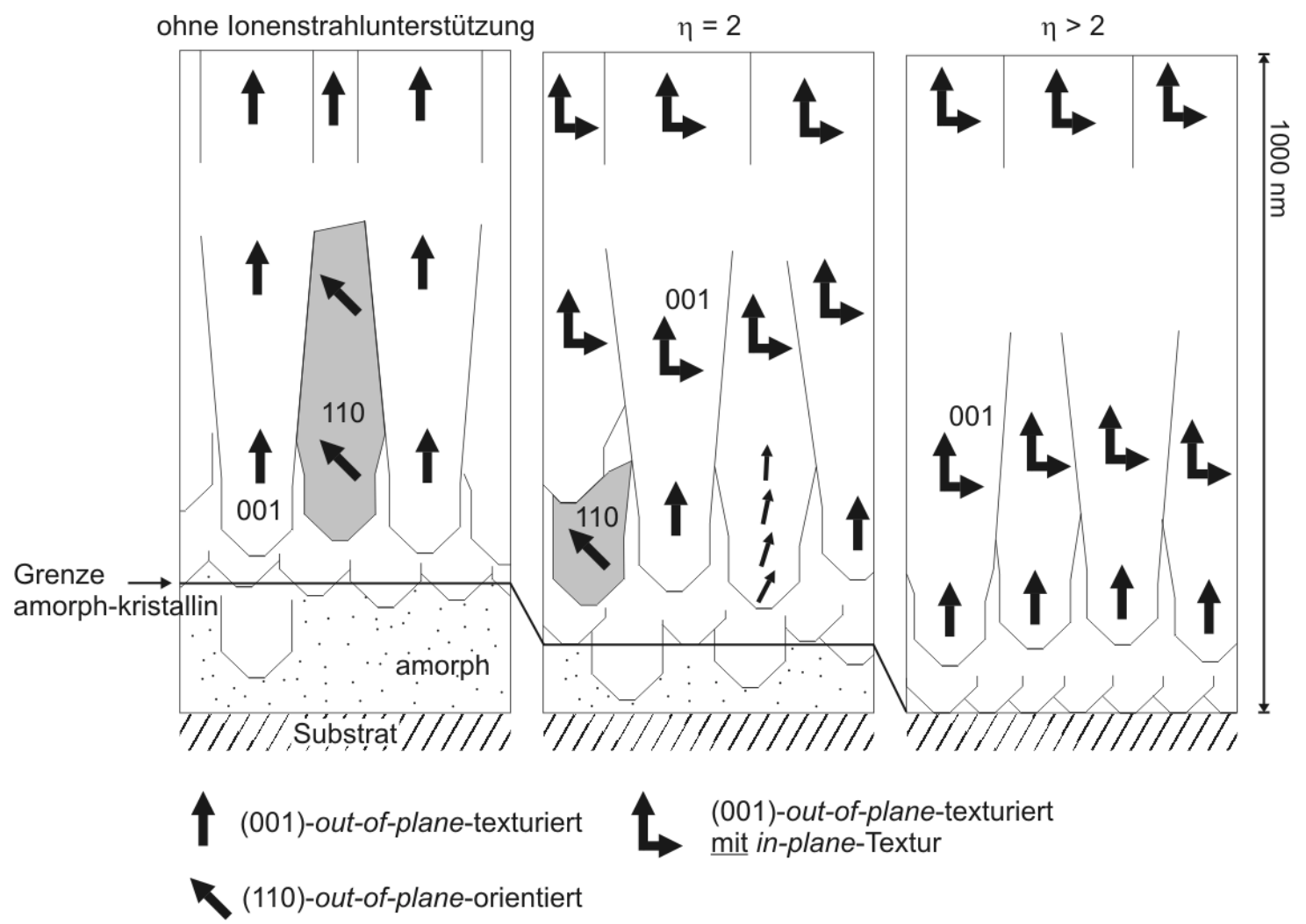

Abbildung 7.8: Schematische Darstellung des Wachstums von YSZ-Filmen, abgeschieden bei verschiedenen $\eta$-Verhältnissen. 
Anfangs bildet sich ein amorpher Film, dessen Dicke mit zunehmendem $\eta$-Verhältnis abnimmt. In YSZ-Filmen, die ohne unterstützenden Ionenbeschuss abgeschieden wurden, kommt es zur homogenen Keimbildung, bei $\eta>0$ ist diese ionenstrahlinduziert. Die Kristallite wachsen weiter und bilden eine kristalline Anfangsschicht, die zu Beginn keine Vorzugorientierung zeigt, wobei (110)- und (001)-Orientierungen verhäuft auftreten. Der Anteil der (110)-Körner sinkt dabei mit zunehmendem $\eta$-Verhältnis und Filmdicke. Das Wachstum von (111)-Körner wird aufgrund von Spannungen in den Filmen unterdrückt. In dieser Phase ist das Ankeimen von neu-orientierten Körnern möglich.

Die nächste Phase ist durch Säulenwachstum geprägt. In ihr bildet sich die (001)-Textur, wobei auch die Anfänge der Bildung einer in-plane-Textur zu beobachten sind. Neben Spannungsselektion und eventuellen Channelings-Prozessen, die zu einem Überwachsen führen, sind auch kontinuierliche Verkippungen der Orientierung von Wachstumssäulen möglich (kleine Pfeile in Abbildung 7.8).

Während sich mit zunehmender Filmdicke die Säulen verbreitern, verbessert sich die Textur. Ab einer, vom $\eta$-Verhältnis abhängigen Filmdicke, verlaufen die Säulengrenzen parallel zur Wachstumsrichtung. Ab dieser Wachstumsphase geschieht die Texturverbesserung nur noch langsam. Die in der Frühphase beobachteten kontinuierlichen Orientierungsänderungen konnte in dieser Wachstumsphase zwar nicht nachgewiesen werden, aber es ist wahrscheinlich, dass dieses der noch einzige ablaufende Prozess ist. Benachbarte Säulen in ca. $1 \mu \mathrm{m}$ dicken IBAD-YSZ-Filmen haben ca. $3^{\circ}$ Orientierungsunterschiede in Wachstumsrichtung, wobei sie in der Ebene um max. $7^{\circ}$ verdreht sind. In dieser Filmdicke existieren auch sehr gestörte Bereiche, die einen entscheidenden Einfluss auf das spätere epitaktische Wachstum des Supraleiters haben können. 


\section{Zusammenfassung}

In dieser Arbeit wurden transmissionselektronenmikroskopische Untersuchungen an bei Raumtemperatur, mittels Ionenstrahlunterstützter Deposition (IBAD) abgeschiedener Yttriumstabilisierter Zirkonoxid-Filme (YSZ) durchgeführt, um so eine detaillierte Beschreibung des Wachstumsmechanismus bei der Ausbildung der biaxialen Textur zu ermöglichen. Dabei wurden die mikroskopischen Ergebnisse mit Röntgenmessungen verglichen.

Für die konventionelle Herstellung von YSZ-Pufferschichten sind die Parameter des unterstützenden Ionenstrahls von entscheidender Bedeutung. Bei einem Einfallswinkel von $55^{\circ}$ und dem Einsatz von Argon-Ionen mit 300 eV wurden die Filme unter verschiedener Beschussintensität des unterstützenden Ionenstrahls abgeschieden, um so Aussagen über den Einfluss des Ionenbeschusses während des Wachstum machen zu können. Das sogenannte $\eta$-Verhältnis lag dabei zwischen 0 (Abscheidung ohne Ionenstrahlunterstützung) und 4 (hohe Intensität des unterstützenden Ionenstrahls). Experimente, bei denen dünne Filme nachträglich mit Ionen beschossen wurden (ITEX), geben Aufschluss über den Einfluss der Ionendosis.

Vor der Deposition wurden die Substrate mittels des Ionenstrahls gereinigt. Diese Reinigung verursacht eine asymmetrische Wellenstruktur auf der Substratoberfläche mit einer Wellelänge von $8-14 \mathrm{~nm}$ und einer Amplitude von ca. 2,5 nm. Diese stellt die für die weitere YSZ-Beschichtung effektive Oberfläche dar.

Die Frühphase des Filmwachstums ist durch eine amorphe Anfangsschicht charakterisiert, deren Dicke mit zunehmendem $\eta$-Verhältnis abnimmt und für $\eta=0$ bis zu $50 \mathrm{~nm}$ betragen kann. Die wenigen Kristallite an der Substratoberfläche keimen bevorzugt an den flacheren Flanken der Wellen an (Abschattungseffekt).

Im Gegensatz zu einer ionenstrahlunterstützten Deposition werden beim nachträglichen Ionenbeschuss keine neuen Kristallzentren erzeugt, da hier die mittlere Korndichte gleich bleibt.

Durch die ionenstrahlinduzierte Keimbildung und das weitere Wachstum der Keime wird eine (poly)kristalline Anfangsschicht gebildet, welche zu Beginn keine Vorzugsorientierung zeigt, wobei (110)- und (001)-Orientierungen dominieren. In dieser frühen Wachstumsphase ist das Ankeimen von neu orientierten Körnern ein häufig beobachteter Prozess. Die Filme weisen hier schon hohe Druckspannungen auf, was anhand von Röntgenmessungen und Beugungsexperimenten gezeigt wurde. Aufgrund dieser Spannungen kann in Filmdicken bis zu ca. $100 \mathrm{~nm}$ die monokline Phase des $\mathrm{ZrO}_{2}$ auftreten. Die Druckspannungen werden als mögli- 
che Ursache der Unterdrückung der (111)-Orientierung und der Ausbildung der (110)- und (001)-Vorzugsorientierungen in der Frühphase des Filmwachstums angesehen.

Röntgenexperimente zeigten, dass die mittels IBAD hergestellten YSZ-Filme eine ausgeprägte (001)-Textur aufweisen. Mit steigender Beschussintensität des unterstützenden Ionenstrahls und zunehmender Filmdicke verringert sich der Anteil von (110)-orientierten Bereichen. In YSZ-Filmen, die ohne Ionenstrahlunterstützung abgeschieden wurden, beobachtet man einen deutlich höheren (110)-Anteil, so dass man erkennen kann, dass der Ionenstrahl das (110)-Wachstum unterdrückt.

Neben dem Ankeimen neuer Körner (diskontinuierliche Orientierungsänderung), konnte in TEM-Querschnittsbildern auch der Prozess der kontinuierlichen Orientierungsänderung beobachtet werden. Die durch Versetzungseinbau bedingte Orientierungsänderung ist dabei sowohl in der Ebene als auch in Wachstumsrichtung möglich.

Bei weiterem Filmwachstum kommt es zur Wachstumsselektion. Die Ausbildung der (001)-Textur in IBAD-YSZ-Filmen, die bei $\eta=2$ abgeschieden wurden, ist ab einer Filmdicke von ca. $300 \mathrm{~nm}$ abgeschlossen. Beugungsexperimente zeigten einen exponentiellen Verlauf der in- und out-of-plane-Textur mit einer leichten Sättigung bei 500 nm Filmdicke. YSZFilme, die ohne Ionenstrahlunterstützung hergestellt wurden, zeigen keine in-plane-Textur. In dieser Wachstumsphase ist das Überwachsen ein häufig beobachteter Prozess.

Das weitere Wachstum geschieht kolumnar. Während sich die Säulen mit zunehmender Filmdicke verbreitern, verbessert sich die Textur. Ab einer vom $\eta$-Verhältnis abhängigen Filmdicke verlaufen die Säulengrenzen parallel zur Wachstumsrichtung, ab hier findet nur noch einer leichte Verbesserung der Textur statt. Die Versetzungsdichte an der Oberseite von $1 \mu \mathrm{m}$ dicken YSZ-Filmen beträgt ca. $10^{11} \mathrm{~cm}^{-2}$. Möglicherweise stellt sich ein Gleichgewicht von Orientierungsverbesserung und Defekteinbau ein. Dieses könnte erklären, warum die Textur nicht beliebig gut wird.

Die mittlere Breite der Säulen an der Oberfläche von $1 \mu \mathrm{m}$ dicken YSZ-Filmen beträgt $100 \mathrm{~nm}$. Benachbarte Säulen haben hier ca. $3^{\circ}$ Orientierungsunterschiede (out-of-plane), wobei sie um bis zu $7^{\circ}$ in der Ebene verdreht sind. Es wurden in diesen Filmdicken auch sehr gestörte Bereiche gefunden, die einen wichtigen Einfluss auf den später epitaktisch abgeschiedenen Supraleiter haben können.

Verschiedene Modellvorstellungen wurden diskutiert, die eine biaxiale Textur durch eine Unterdrückung von fehlorientierten Körnern beschreiben. Aufgrund der Anisotropie der Ätzrate können unterschiedlich ausgerichtete Körner verschieden stark vom Ionenstrahl abgetragen werden. Es kommt zu einem Höhenvorteil der YSZ-Körner, deren <111>-Richtung parallel zum unterstützenden Ionenstrahl verlaufen, da diese Ebenen aufgrund von Channelingseffekten weniger stark abgetragen werden. 
Die Untersuchungen zum Einfluss der Intensität des unterstützenden Ionenstrahls auf die einzelnen Wachstumsphasen von IBAD-YSZ-Filmen haben gezeigt, dass es prinzipiell denkbar ist, die einzelnen Phasen durch verschiedene Beschussintensitäten getrennt zu optimieren, um so ein schnellere Texturausbildung zu erhalten. 


\section{Literaturverzeichnis}

$\boldsymbol{A}$

\section{Akimov et al., 1994}

G. Y. Akimov, V. M. Timchenko and I. V. Gorelik

Physics of the Solid State, 36 (1994), S. 1906

\section{Alexander, 1997}

H. Alexander

Physikalische Grundlage der Elektronenmikroskopie

B.G. Teubner Stuttgart, 1997

\section{B}

\section{Bardal et al., 1994}

A. Bardal, Th. Matthée, J. Wecker and K. Samwer Journal of Applied Physics, 75, 6 (1994)

\section{Bauer et al., 1999}

M. Bauer, R. Semerad, H. Kinder, J. Wiesmann, J. Dzick, H. C. Freyhardt IEEE Transactions on Applied Superconductivity, 9 (1999), S. 2244

\section{Baufeld et al., 1998}

B. Baufeld, D. Baither, M. Bartsch and U. Messerschmidt Physica Status Solidi (a), 166, 127 (1998), S. 127

\section{Behner, 1992}

H. Behner, J. Wecker and B. Heines

Edited by L. Correra (Elsevier, Amsterdam), 1992

\section{Behrisch, 1981}

R. Berisch (Hrsg.)

Topics in Applied Physics, 47, Springer Verlag (1981)

\section{Betz et al., 1997}

V. Betz, B. Holzapfel, G. Sipos, W. Schmidt, N. Mattern and L. Schultz Applied Superconductivity, Inst. Phys. Conf. Ser. 158 (1997), S. 1081

\section{Bradley und Harper, 1988}

R. M. Bradley and J. M. E. Harper

Journal of Vacuum Science \& Technology A, 6 (1988), S. 2390 
C

\section{Christensen und Carter, 1998}

A. Christenden and E. A. Carter

Physical Review B, 58, 12 (1998), S. 8050

D

D’Heurle, 1970

F. M. D'Heurle

Metallurgical Transactions, 1 (1970), S. 725

D’Heurle und Harper, 1989

F. M. D'Heurle and J. M. E. Harper

Thin Solid Films, 171 (1989), S. 81

\section{Dimos et al., 1990}

D. Dimos, P. Chaudhari and J. Mannhart

Physical Review B, 41 (1990), S. 4038

\section{Dong et al., 1998}

L. Dong und D. J. Srolovitz

Journal of Applied Physics, 84, 9 (1998), S. 5261

\section{Dong et al., 1999}

L. Dong und D. J. Srolovitz

Applied Physics Letters, 75, 4 (1999), S. 584

\section{Dzick, 1996}

J. Dzick

Diplomarbeit, Institut für Metallphysik, Universität Göttingen (1996)

\section{Dzick, 2000}

J. Dzick

Dissertation, Institut für Metallphysik, Universität Göttingen (2000)

\section{$\boldsymbol{E}$}

\section{Edington, 1975}

J. W. Edington

Interpretation of Transmission Electron Micrographs

Philips Technical Libraray, Monographs in Practical Electron Microscopy in Materials Science 3 (1975), S. 80

\section{EMS Online}

P.-H. Jouneau und P. Stadelmann

Centre Interdépartemental de Microscopie Electronique, EPFL, Lausanne http://cimesg1.epfl.ch/CIOL/ems.html 


\section{Ensinger, 1995}

W. Ensinger

Nuclear Instruments and Methods in Physics B, 106, 142 (1995)

$\boldsymbol{F}$

Freyhardt et al., 1996

H. C. Freyhardt, J. Hoffmann, J. Wiesmann, J. Dzick, K. Heinemann, A. Isaev, F. GarcíaMoreno, S. Sievers and A. Usoskin

IEEE Transaction on Applied Superconductivity 7 (1996), S. 1426

\section{Friedel, 1964}

J. Friedel

Dislocations

Pergamon Press, International Series of Monographs in Solid State Physics, 3 (1964), S. 55

$\underline{G}$

\section{Goyal et al., 1996}

A. Goyal, D. P. Norton, J. D. Budai, M. Paranthaman, E. D. Specht, D. M. Kroeger, D. K. Christen, Q. He, B. Saffian, F. A. List, D. F. Lee, P. M. Martin, C. E. Klabunde, E. Hartfield and V. K. Sikka Applied Physics Letters, 69 (1996), S. 1795

\section{$\boldsymbol{H}$}

\section{Heuer et al., 1985}

A. H. Heuer, S. Kraus-Lanteri, P. A. Labun, V. Lanteri and T. E. Mitchell Ultramicroscopy, 18 (1985), S.335

\section{Hoffmann, 1990}

D. W. Hoffmann

Journal of Vacuum Science \& Technology A, 8 (1990), S. 3707

\section{Hund, 1951}

F. Hund

Zeitschrift für Elektrochemie, 55 (1951), S.363

\section{$\boldsymbol{I}$}

\section{Iijima et al., 1991}

Y. Iijima, N. Tanabe, Y. Ikeno, and O. Kohno

Physica C, 185 (1991), S. 1959

\section{Iijima et al., 1998}

Y. Iijima, M. Hosaka, N. Tanabe, N. Sadakata, T. Saitoh, O. Kohno and K. Takeda Journal of Materials Research, 13, 11 (1998), S. 3106 


\section{Iijima et al., 1998a}

Y. Iijima, M. Hosaka, N. Tanabe, N. Sadakata, T. Saitoh, O. Kohno and K. Takeda Applied Superconductivity, Special Issue „YBCO Conductor Development for Large Scale Applications“, 4, 10-11 (1998)

\section{$\boldsymbol{K}$}

\section{Kaufman, 1974}

H. R. Kaufman

In: Advances in Electronics and Electron Physics 36

Hrsg. L. Marton (1974), S. 265

\section{Kaufman et al., 1982}

H. R. Kaufman, J. J. Cuomo and J. M. E. Harper

Journal of Vacuum Science \& Technology A, 21 (1982), S. 725

\section{Kaufman et al., 1998}

D. Y. Kaufman, P. M. DeLuca, T. Tsai and S. A. Barnett

Journal of Vacuum Science \& Technology A, 17 (1999), S. 2826

\section{Kung et al., 2001}

H. Kung, P. Arendt, S. Foltyn, R. Groves, T. Holesinger, Q. Jia, V. Matias, E. Peterson, L. Emmert, R. DePaula, P. Dowden, Y. Coulter and L. Stan

Superconductivity for Electric Systems, Annual Peer Review, August 1-3, 2001, http://www.lanl.gov/orgs/mst/stc/docs/2001IBAD.pdf

\section{$\boldsymbol{L}$}

\section{Li et al., 2002}

T. Q. Li, S. Noda, Y. Tsuji, T. Ohsawa and H. Komiyama

Journal of Vacuum Science \& Technology A, 20 (2002)

\section{$M$}

\section{Mao et al., 1997}

Y. J. Mao, C. X. Ren, J. Yuan, F. Zhang, X. H. Liu and S. C. Zou

Journal of Vacuum Science \& Technology A, 15 (1997), S. 2678

\section{McCullough und Trueblood, 1959}

J. D. McCullough and K. N. Trueblood

Acta Crystallographica, 12, (1959), S. 507

\section{Meingast et al., 1991}

C. Meingast, O. Kraut, T. Wolf, H. Wühl, A. Erb, G. Müller-Vogt

Physical Review Letters, 67 (1991), S. 1634

\section{Miteva et al., 1998}

V. A. Miteva and I. R. Chakarov

Vacuum, 51, 267 (1998) 


\section{Mizutani, 1995}

T. Mizutani

Journal of Non-Crystalline Solids, 181 (1995), S. 123

Molodetsky et al., 2000

I. Molodetsky, A. Navrotsky, M. J. Paskowitz, V. J. Leppert und S. H. Risbud Journal of Non-Crystalline Solids, 262 (2000), S. 106

\section{$N$}

Navez et al., 1962

M. Navez, C. Sella et D. Chaperot

Comptes Rendus de l'Académie des Sciences., 254 (1962), S. 240

\section{Nastasi et al., 1996}

M. Nastasi, J. W. Mayer and J. K. Hirvonen

Ion-Solid interactions: fundamentals and applications

Cambridge University Press, 1996

\section{$\boldsymbol{P}$}

\section{Pelleg et al., 1991}

J. Pelleg, L. Z. Zevin and S. Lungo

Thin Solid Films, 197, 117 (1991)

\section{$\boldsymbol{R}$}

\section{Read und Shockley, 1950}

W. T. Read und W. Shockley

Physical Review, 78 (1950), S. 275

\section{Reade et al., 2002}

R. P. Reade, P. Berdahl und R. E. Russo

Applied Physics Letters, 80, 8 (2002), S. 1352

\section{Ressler et al., 1997a}

K. G. Ressler, N. Sonnenberg und M. J. Cima

Journal of the American Ceramic Society, 80 (1997), S. 2637

\section{Ressler et al., 1997b}

K. G. Ressler, N. Sonnenberg und M. J. Cima

IEEE Trans. Appl. Supercond., 7 (1997), S. 1432

\section{$S$}

\section{Scott, 1975}

M. G. Scott

Journal of Material Science, 10 (1975), S. 3682 


\section{Scubican et al., 1978}

R. Scubican, R. C. Hink, S. P. Ray

Journal of the American Ceramic Society, 61 (1978), S. 17

\section{Sievers, 2002}

S. Sievers

persönliche Mitteilung (2002)

\section{Sonnenberg et al., 1993}

N. Sonnenberg, A. S. Lomgo, M. J. Cima, B. P. Chang, K. G. Ressler, P. C. McIntyre and Y. P. Liu

Journal of Applied Physics, 74 (1993), S. 1027

\section{Spence, 1988}

J. Spence

Experimental High-Resolution Electron Microscopy

Oxford University Press, New York, Oxford (1988)

\section{Spinella et al., 1991}

C. Spinella, L. Lombardo and S. U. Campisano

Physical Review Letters, 66, 8 (1991)

\section{$T$}

\section{Thornton et al., 1989}

J. A. Thornton and D. W. Hoffmann

Thin Solid Films, 171 (1989), S. 5

\section{Thiele et al., 2002}

K. Thiel, s. Sievers, C. Jooss, J. Hoffmann and H. C. Freyhardt

Journal of Material Research, eingereicht (2002)

$\underline{V}$

van der Drift, 1968

A. van der Drift

Philips Research. Reports, 22, (1968), S. 267

W

Wang et al., 1997

C. P. Wang, K. B. Do, M. R. Beasley, T. H. Geballe and R. H. Hammond Applied Physics Letters, 71 (1997), S. 2955

\section{Wiesmann, 1994}

J. Wiesmann

Diplomarbeit, Institut für Metallphysik (1994)

\section{Wiesmann, 1998}

J. Wiesmann

Dissertation, Institut für Materialphysik, Universität Göttingen (1998) 


\section{Williams und Carter, 1996}

D. B. Williams und C. B. Carter

Transmission electron microscopy: A Textbook for Materials Science

Plenum Press, New York and London (1996) S. 291

\section{Windischmann, 1987}

H. Windischmann

Journal of Applied Physics, 62 (1987), S. 1800

\section{Windischmann, 1992}

H. Windischmann

Critical Reviews in Solid State and Materials Sciences, 17 (1992), S. 547

\section{Window, 1993}

B. Window

Journal of Vacuum Science \& Technology A, 11 (1993), S. 1522

\section{Wu et al., 1995}

X. D. Wu, S. R. Foltyn, P. N. Arendt, W. R. Blumenthal, I. H. Campbell, J. D. Cotton, J. Y. Coulter, W. L. Hults, M. P. Maley, H. F. Safar and J. L. Smith Applied Physics Letters, 67 (1995), S. 2397

$\boldsymbol{Y}$

\section{Ying et al., 1996}

F. Ying, R. W. Smith and D. J. Srolovitz

Applied Physics Letters, 69 (1996), S. 3007

\section{Yu et al., 1985}

L. S. Yu, J.W. Gerlach, T. Kraus and B. Rauschenbach Applied Physics Letters, 47 (1985), S. 932

\section{Yu et al., 1986}

L. S. Yu, J. M. E. Harper, J. J. Cuomo and D. A. Smith Journal of Vacuum Science \& Technology A, 4 (1986), S. 443

\section{$\boldsymbol{Z}$}

\section{Ziegler et al., 1985}

J. F. Ziegler, J. P. Biersack and U. Littmark

The Stopping and Range of Ions in Solids, Pergamon Press, New York (1985)

\section{Ziegler und Biersack, 2000}

J. F. Ziegler und J. P. Biersack

Software SRIM-2000

http://www.srim.org 


\section{Danksagung}

Herrn Prof. Dr. H. C. Freyhardt danke ich für die Ermöglichung und Förderung dieser Arbeit.

Frau Dr. S. Sievers und Herrn Dr. J. Hoffmann möchte für die vielen Anregungen und Diskussionen sowie die konstruktiven Korrekturvorschläge beim Abschluss dieser Arbeit sehr danken.

Herrn Dr. C. Jooss danke ich für die Unterstützung, nicht nur bei Physikfragen.

Den Mitarbeitern der Sputter-Gruppe Frau K. Thiele, Frau C. Brandt, Herrn K. Herweg und Herrn S. Dreyer danke ich für die anregenden Diskussionen und gute Zusammenarbeit.

Frau Dr. C. Borchers danke ich für die vielen Ratschläge und Anregungen.

Bei Herrn Dr. M. Seibt bedanke ich mich für die Einweisung und Unterstützung bei der Bedienung des ,200ers“.

Herrn D. Jockel danke ich für die ständige Diskussionsbereitschaft.

Für die Überlassung der Proben danke ich Herrn Dr. J. Dzick.

Herrn M. Hahn danke ich für seinen unermüdlichen Einsatz für die Funktionstüchtigkeit des Elektronenmikroskops und der Ionenätze.

Bei K. Sturm bedanke ich mich für die vielen langen Diskussionsabende.

Herrn J. Bankmann danke ich für die vielen Diskussionen vor, während und nach dem Kickern.

Mein Dank gilt insbesondere allen Mitarbeiterinnen und Mitarbeitern des Instituts für Materialphysik und des Zentrums für Funktionswerkstoffe für die freundschaftliche Arbeitsatmosphäre.

Bei der Berliner-Ungewitter-Stiftung bedanke ich mich für die finanzielle Hilfe am Ende meiner Doktorarbeit.

$\mathrm{Zu}$ guter Letzt danke ich meinen Eltern und Freunden, die mich während der Doktorarbeit unterstützt und begleitet haben. 


\section{Lebenslauf}

\section{Persönliche Daten:}

Name:

Nationalität:

Geburtstag:

Geburtsort:

Schulausbildung:

$1974-1978$

1978 - 1989

10. Mai 1989

Zivildienst:

$1989-1991$

\section{Studium:}

WS 1991

12. August 1994

WS 1994 - WS 1996

30. Oktober 1997

SS 1998

\author{
Lars-Oliver Kautschor \\ deutsch \\ 3. Januar 1968 \\ Stade (Niedersachsen)
}

Luther-Schule, Paderborn

Gymnasium Schloß-Neuhaus

Abitur

Zivildienst im Heim Freundeskreis Ochsenzoll e.V., Hamburg

Aufnahme des Physikstudiums an der Universität Hamburg

Diplomvorprüfung Physik

Hauptstudium Physik an der Universität Göttingen

Diplomprüfung Physik

Diplomarbeit bei Herrn Prof. Dr. H. C. Freyhardt am Institut für Metallphysik mit dem Thema:

Elektronenmikroskopische Untersuchungen der Defektstruktur unverformter und verformter schmelztexturierter YBCO-Monolithe

Einschreibung in den Aufbaustudiengang Physik an der Universität Göttingen

Beginn der Dissertation in der Arbeitsgruppe von

Herrn Prof. Dr. H. C. Freyhardt am Institut für Materialphysik

Beschäftigungen:

$1.1 .1998-30.6 .2002$
Wiss. Mitarbeiter am Institut für Materialphysik 